\title{
Kernel-Based Inference in Time-Varying Coefficient Cointegrating Regression
}

\author{
Degui Li, Peter C. B. Phillips ${ }^{\dagger}$ and Jiti Gao ${ }^{\ddagger}$
}

April 12, 2019

\begin{abstract}
This paper studies nonlinear cointegrating models with time-varying coefficients and multiple nonstationary regressors using classic kernel smoothing methods to estimate the coefficient functions. Extending earlier work on nonstationary kernel regression to take account of practical features of the data, we allow the regressors to be cointegrated and to embody a mixture of stochastic and deterministic trends, complications which result in asymptotic degeneracy of the kernel-weighted signal matrix. To address these complications new local and global rotation techniques are introduced to transform the covariate space to accommodate multiple scenarios of induced degeneracy. Under regularity conditions we derive asymptotic results that differ substantially from existing kernel regression asymptotics, leading to new limit theory under multiple convergence rates. For the practically important case of endogenous nonstationary regressors we propose a fully-modified kernel estimator whose limit distribution theory corresponds to the prototypical pure cointegration case (i.e., with exogenous covariates), thereby facilitating inference using a generalized Wald-type test statistic. These results substantially generalize econometric estimation and testing techniques in the cointegration literature to accommodate time variation and complications of co-moving regressors. Finally, Monte-Carlo simulation studies as well as an empirical illustration to aggregate US data on consumption, income, and interest rates are provided to illustrate the methodology and evaluate the numerical performance of the proposed methods in finite samples.
\end{abstract}

Keywords: Cointegration; FM-kernel estimation; Generalized Wald test; Global rotation; Kernel degeneracy; Local rotation; Super-consistency; Time-varying coefficients.

Abbreviated Title: Kernel Inference in Cointegrating Regression

*University of York

'Yale University, University of Auckland, Southampton University, and Singapore Management University

${ }^{\ddagger}$ Monash University 


\section{Introduction}

Many time series that are encountered in economics and finance are well known to exhibit nonstationary characteristics such as the random wandering behavior of financial asset prices and the secular growth components in aggregate time series data that indicate the presence of some form of deterministic drift. Following the work of Phillips and Durlauf (1986), Engle and Granger (1987), Park and Phillips (1988, 1989), Phillips $(1988,1991)$ and Johansen (1991), substantial investments have been made in econometric methodology to take account of these characteristics in linear and log linear cointegrating regression estimation and inference.

Notwithstanding this body of work many practical implementations reveal that parametric linear cointegration models are often rejected by the data even when there is evident co-movement among the trending series. Acknowledgement of this weakness has led to the recent development of econometric methodology for treating various nonlinear and nonparametric cointegrating models (Park and Phillips, 2001; Karlsen, Myklebust and Tjøstheim, 2007; Cai, Li and Park, 2009; Wang and Phillips, 2009a,b; Xiao, 2009; Gao and Phillips, 2013; Li et al, 2017; Phillips, Li and Gao, 2017). For the important case of multivariate integrated covariates, much of this nonparametric research on nonlinear cointegration excludes possible co-movement among the regressors and the presence of deterministic drift. Such restrictions simplify asymptotic theory but limit applicability of the methods to time series without the commonly occuring characteristics that produce co-movement over time and asymptotic degeneracies in the signal matrix.

The primary goal of the present paper is to relax these restrictions by allowing more flexible structures among the covariates, to develop kernel regression asymptotics for a general class of models that accommodate these key features in the data, and to provide inferential machinery that enables convenient estimation and inference in practical work. In developing these methods, our main focus of attention is a multiple regression model with time-varying coefficients of the following form

$$
Y_{t}=\beta_{t}^{\prime} X_{t}+e_{t 0}, \quad t=1, \cdots, T,
$$

where $\beta_{t}:=\beta(t / T)$ is a $d$-dimensional vector of coefficients which varies over time, $\beta(\cdot)$ is a d-dimensional vector of functions, $\left\{X_{t}\right\}$ is a d-dimensional nonstationary process, and $\left\{e_{t 0}\right\}$ is a stationary random error process. The paper studies three generating structures on $X_{t}$ of increasing complexity: (i) $X_{t}$ is cointegrated with $d_{0}$ cointegrating vectors and no deterministic trend where $0 \leqslant d_{0} \leqslant d-1$; (ii) $X_{t}$ involves a mixture of deterministic and stochastic trends but without any cointegrating structure; and (iii) $X_{t}$ is cointegrated and has deterministic trend components. Scenario (iii) is the most general and combines the complications of (i) and (ii). In view of the special technical difficulties involved, it is convenient to treat these generating structures for the regressors $X_{t}$ individually at first, leading ultimately to a complete set of asymptotics for coefficient function estimation and inference in models of the form (1.1) with time-varying functional coefficients and co-moving endogenous regressors.

Model (1.1) is motivated by the need for a flexible framework that captures structural change via temporal evolution in the functional coefficients in regressions with nonstationary data. The formulation usefully circumvents the curse of dimensionality problems that commonly arise in nonparametric regression estimation when the dimension of the covariates is large and that are known to be exacerbated in the nonstationary nonparametric case due to slower convergence rates (Wang and Phillips, 2009a). The modelling framework (1.1) includes and extends many linear and nonlinear cointegration models that have been extensively 
studied in the literature. For instance, in the constant coefficient case where $\boldsymbol{\beta}_{t}=\boldsymbol{\beta}$, model (1.1) is a multiple linear regression with integrated regressors in which scenarios (i)-(iii) above may be present in practical work and for which asymptotic linear regression theory was developed in early work by Park and Phillips (1988, 1989), Phillips $(1988,1995)$, and Toda and Phillips (1993). When $X_{t}$ is not cointegrated and no deterministic drift is involved, model (1.1) reduces to the model studied in Park and Hahn (1999) and Phillips, Li and Gao (2017) where sieve estimation and kernel-based estimation techniques were analyzed, respectively. When the nonstationary regressors $X_{t}$ are cointegrated, there exist certain linear combinations of $X_{t}\left(\right.$ if $d_{0} \geqslant 1$ ) which can lower the order of integration, leading to the presence of a stationary process component in the regressors. It follows that our modelling framework also relates to work on time-varying coefficient models with stationary (or locally stationary) regressors (c.f., Robinson, 1989; Cai, 2007; Zhou and Wu, 2010; Chen and Hong, 2012; Vogt, 2012; Zhang and Wu, 2012; Giraitis, Kapetanious and Yates, 2014), and may be regarded as an extension of that work to accommodate nonstationary and trending regressor components. The upshot is that the results obtained in the present paper have wide potential applicability to economic time series with stationary, trend stationary, co-moving, and stochastically nonstationary components.

The paper applies standard Nadaraya-Watson kernel methods to estimate the coefficient function $\beta(\cdot)$ in the presence of a complicating structure of co-moving and co-trending regressors that raises significant challenges in the development of a limit theory for kernel estimation and inference. The technical challenges may be explained in a heuristic manner as follows. The central difficulty arises from the multiple asymptotic singularities that feature in the kernel-weighted signal matrix - the random matrix that carries the kernel weights and appears in the denominator of the usual kernel estimator. Rotation techniques are used to conform the covariate space to accommodate signals of various orders in developing the asymptotic theory. These techniques extend those that were developed and are now commonly used in the nonstationary linear regression literature (Phillips, 1988; Park and Phillips, 1988, 1989) to the kernel regression environment where both global and local rotators are required. If the regressors are cointegrated, a global rotation technique is applied to separate out the stationary components and nonstationary components, which carry the associated signals in kernel estimation with differing strengths. In the multivariate regressor case, the kernel-weighted random matrix associated with the nonstationary covariate components may have dimension greater than unity, inducing a further signal degeneracy that we refer to as local degeneracy throughout the paper.

When the nonstationary components have only stochastic trends, time-varying coefficient kernel regression naturally concentrates attention on a particular time coordinate of the partial sum process and, in doing so, the associated stochastic process limit process. This focus on a local time coordinate produces a limiting kernel signal matrix of deficient rank unity. On the other hand, when the stochastic trends are themselves asymptotically majorized by deterministic linear trends, the nonstationary components become dominated asymptotically by these linear trends, which reduces asymptotic variability across component variables and leads to further degeneracy in the asymptotics. The local rotation approach used in the present paper addresses this further degeneracy in the nonstationary components and applies whether these components are dominated by stochastic or deterministic trends.

This rotation geometry enables the development of a full asymptotic distribution theory for nonstationary kernel estimation under general regularity conditions. The main results reveal multiple convergence rates in the different directions associated with the rotations. These directions include the usual stationary regressor nonparametric convergence rate $(\sqrt{T h})$, a type 1 super-consistency rate $(T \sqrt{h})$, a type 2 super-consistency 
rate $(T h)$, and a type 3 rate $(T \sqrt{T h})$ in the direction of the deterministic linear trends. When nonstationary regressors in time-varying coefficient models are endogenous, second-order bias terms are present in the kernel regression limit theory, analogous to the endogeneity bias that occurs in linear cointegrating regression. Although such bias does not affect convergence rates in the asymptotics, it does influence finite sample performance and inference with kernel methods. To address this endogeneity bias, a fully-modified (FM) kernel estimator is developed for which the asymptotic theory corresponds to the pure (exogenous regressor) cointegration case.

A further contribution of the paper is to develop inferential methods for the time-varying coefficient functions in model (1.1). Two different null hypotheses on the coefficient functions are considered, which allow for universal restrictions (that is, restrictions that apply uniformly over time) and local restrictions (that is, restrictions that apply pointwise at some specific time-point corresponding to some sample fraction of the data). Generalized Wald-type test statistics are constructed to test these hypotheses. The limit theory for these tests is developed under both full rank and deficient rank conditions on the covariance structure of the restricted function coefficients, thereby accommodating potential implications for rank arising from signal matrix degeneracies in kernel estimation. The resulting asymptotics involve two types of chi-square limit distributions and possibly divergent degrees of freedom. This limit theory substantially extends existing work on inference in linear cointegrating regressions (particularly, Park and Phillips, 1988, 1989; Toda and Phillips, 1993; Phillips, 1995) to the nonlinear cointegrating model setting.

These contributions combine to bring the limit theory for functional nonparametric nonstationary regression to a similar level of generality as the earlier limit theory for linear cointegating regression, allowing for multiple forms of asymptotic degeneracies in the regressor space and delivering asymptotically chi-square tests that enable inference in nonlinear co-moving systems with multiple covariates under endogeneity. The methods of the paper therefore apply widely and provide a convenient framework for investigators to test hypotheses concerning time evolution and stability in regression coefficients in nonstationary time series environments.

The rest of the paper is organized as follows. Section 2 describes the kernel estimation approach, provides assumptions, develops the double-rotation technique, and derives asymptotic theory when the regressors are cointegrated. Section 3 generalizes the structure and theory to the case where the regressors have a mix of stochastic and deterministic trends and the case when the regressors are cointegrated with deterministic trends. Section 4 introduces the FM kernel estimator and establishes its limit distribution theory. Section 5 explores methods of inference on the coefficient functions. Section 6 reports extensive simulation studies to evaluate the finite sample properties of the proposed methods in relation to the developed asymptotic theory. Section 7 provides an empirical illustration to aggregate US data on consumption, income and interest rates. Section 8 concludes the paper. Proofs of the main asymptotic results and proofs of some supplementary results are given in Appendices A and B, respectively, which are available in the online supplementary document (Li, Phillips and Gao, 2019). Throughout the paper, we use " $\Rightarrow$ " and " $\rightarrow p$ " to denote weak convergence (or convergence in distribution) in the relevant space and convergence in probability, respectively. Let $\|\cdot\|$ be the Frobenius norm of a matrix or the Euclidean norm of a vector, and let $\mathbf{A}^{+}$denote the Moore-Penrose generalised inverse of a matrix $\boldsymbol{A}$ which becomes the conventional inverse $A^{-1}$ if $A$ is invertible. 


\section{Kernel estimation with cointegrated regressors}

In this section, we use kernel smoothing to estimate the coefficient functions in model (1.1) when the nonstationary integrated regressors are cointegrated. We study the effects of the resulting asymptotic signal degeneracy, and introduce rotation techniques to derive the limit theory of the kernel estimates.

\subsection{Model estimation and assumptions}

Smoothness conditions on the coefficient function $\beta(\cdot)$ permit local approximation $\beta(z) \approx \beta\left(z_{0}\right)$ for $z$ in any small neighborhood of $z_{0} \in(0,1)$, which motivates Nadaraya-Watson-type local level regression estimation of $\beta\left(z_{0}\right)$ in (1.1) according to the formula

$$
\widehat{\boldsymbol{\beta}}\left(z_{0}\right)=\left[\sum_{\mathrm{t}=1}^{\mathrm{T}} \mathrm{X}_{\mathrm{t}} \mathrm{X}_{\mathrm{t}}^{\prime} \mathrm{K}\left(\frac{\mathrm{t}-\mathrm{T} z_{0}}{\mathrm{Th}}\right)\right]^{+}\left[\sum_{\mathrm{t}=1}^{\mathrm{T}} \mathrm{X}_{\mathrm{t}} \mathrm{Y}_{\mathrm{t}} \mathrm{K}\left(\frac{\mathrm{t}-\mathrm{T} z_{0}}{\mathrm{Th}}\right)\right]=: \Lambda_{\mathrm{T}}^{+}\left(z_{0}\right) \Delta_{\mathrm{T}}\left(z_{0}\right),
$$

where $\mathrm{K}(\cdot)$ is some kernel function, and $\mathrm{h}$ is a bandwidth which tends to zero as the sample size $T$ tends to infinity. While the present paper concentrates on this particular kernel estimation method, other kernelbased methods such as local linear smoothing or local polynomial smoothing approaches (Fan and Gijbels, 1996) may be used in the same way and the methods given here may be suitably modified to accommodate these approaches with similar asymptotic results (c.f., Remark 4 in Section 3.1).

The notation [.] ${ }^{+}$in (2.1) signifies the use of a Moore-Penrose inverse in the case of a singular matrix. Provided the sample size $T$ is large enough and depending on the shape of the particular kernel $K(\cdot)$, the matrix $\left[\sum_{t=1}^{T} X_{t} X_{t}^{\prime} K\left(\frac{t-T z_{0}}{T h}\right)\right]$ will be positive definite almost surely in finite samples just as the sample moment matrix $\sum_{t=1}^{T} X_{t} X_{t}^{\prime}$ itself, in which case use of the Moore Penrose inverse is unnecessary. On the other hand, as analyzed below, in the limit as $T \rightarrow \infty$ the standardized form of the matrix $\left[\sum_{t=1}^{T} X_{t} X_{t}^{\prime} K\left(\frac{t-T z_{0}}{T h}\right)\right]$ is typically singular asymptotically. However, when transformed in certain directions and suitably restandardized, this matrix will be positive definite and these rotations of the regressor space facilitate the asymptotic analysis, as we now demonstrate. The approach is analogous to the one developed in Park and Phillips $(1988,1989)$, but in the present case, random rotation matrices are typically needed.

We commence our analysis with the case where the multivariate integrated regressors $X_{t}$ are cointegrated with $d_{0}$ cointegrating vectors, $0 \leqslant d_{0} \leqslant d-1$. Letting $d_{1}=d-d_{0}$, there exists a $d \times d$ orthogonal matrix $\mathbf{H}=\left(\mathbf{H}_{1}, \mathbf{H}_{2}\right)$ such that

$$
\mathbf{H}_{1}^{\prime} X_{\mathrm{t}}=e_{\mathrm{t} 1}, \Delta\left(\mathbf{H}_{2}^{\prime} \mathrm{X}_{\mathrm{t}}\right)=\mathbf{H}_{2}^{\prime}\left(\Delta \mathrm{X}_{\mathrm{t}}\right)=e_{\mathrm{t} 2}
$$

where the sizes for $\mathrm{H}_{1}$ and $\mathrm{H}_{2}$ are $\mathrm{d} \times \mathrm{d}_{0}$ and $\mathrm{d} \times \mathrm{d}_{1}$, respectively, $\Delta$ denotes the first-order difference operator, and $\left(e_{\mathrm{t} 1}^{\prime}, e_{\mathrm{t} 2}^{\prime}\right)^{\prime}$ is stationary with $e_{\mathrm{t} 1}$ being $\mathrm{d}_{0}$-dimensional and $e_{\mathrm{t} 2}$ being $\mathrm{d}_{1}$-dimensional. In view of (2.2), a rotation of the regressor space conveniently separates out the stationary and nonstationary components of the covariates in model (1.1). The transformation matrix $\mathbf{H}$ is not unique and the rank of the cointegrating space $d_{0}$ together with the associated directions of cointegration that are embodied in the submatrix $\mathbf{H}_{1}$ are generally unknown a priori. We emphasize that knowledge of $d_{0}$ and $\mathbf{H}_{1}$ are not needed for application of (2.1) and the methods of the present paper, including the asymptotic results, can be used in practical work without such knowledge, although there are of course well-known parametric and nonparametric methods of testing to determine $d_{0}$ and procedures to estimate $\mathbf{H}_{1}$ in the existing literature (e.g., Johansen, 
1991; Phillips, 1996; Cheng and Phillips, 2009). The following example illustrates the formulation (2.2) for a simple cointegrated vector autoregression (VAR) model with general stationary errors (c.f., Cheng and Phillips, 2009).

EXAMPLE 1. Define the model

$$
\Delta X_{\mathrm{t}}=\alpha \gamma^{\prime} \mathrm{X}_{\mathrm{t}-1}+v_{\mathrm{t}}
$$

where $\left\{v_{\mathrm{t}}\right\}$ is a covariance stationary time series with mean zero, $\alpha$ and $\gamma$ are $d \times d_{0}$ constant matrices with rank $d_{0}$, and $I_{d_{0}}+\gamma^{\prime} \alpha$ has latent roots inside the unit circle. Let $\gamma_{\perp}$ be a $d \times\left(d-d_{0}\right)$ full rank matrix complement to $\gamma$ so that $\gamma^{\prime} \gamma_{\perp}=O_{d_{0} \times d_{1}}$ where $d_{1}=d-d_{0}$ and $O_{r \times s}$ is an $r \times s$ null matrix, and define

$$
\mathbf{H}_{1}^{\prime}=\left(\boldsymbol{\gamma}^{\prime} \boldsymbol{\gamma}\right)^{-1 / 2} \boldsymbol{\gamma}^{\prime}, \mathbf{H}_{2}^{\prime}=\left(\boldsymbol{\gamma}_{\perp}^{\prime} \boldsymbol{\gamma}_{\perp}\right)^{-1 / 2} \boldsymbol{\gamma}_{\perp}^{\prime}
$$

so that $\mathbf{H}=\left(\mathbf{H}_{1}, \mathbf{H}_{2}\right)$ is orthogonal. Following standard arguments (Johansen, 1991; Phillips, 1995), the VAR model (2.3) falls into the framework of (2.2) with transforms

$$
\mathbf{H}_{1}^{\prime} X_{\mathrm{t}}=\left(\gamma^{\prime} \gamma\right)^{-1 / 2}\left(\mathbf{I}_{\mathrm{d}_{0}}+\gamma^{\prime} \boldsymbol{\alpha}\right) \gamma^{\prime} X_{\mathrm{t}-1}+\left(\gamma^{\prime} \gamma\right)^{-1 / 2} \gamma^{\prime} v_{\mathrm{t}}=: e_{\mathrm{t} 1}
$$

and

$$
\Delta\left(\mathbf{H}_{2}^{\prime} X_{\mathrm{t}}\right)=\left(\gamma_{\perp}^{\prime} \gamma_{\perp}\right)^{-1 / 2} \gamma_{\perp}^{\prime} \alpha \gamma^{\prime} X_{\mathrm{t}-1}+\left(\gamma_{\perp}^{\prime} \gamma_{\perp}\right)^{-1 / 2} \gamma_{\perp}^{\prime} v_{\mathrm{t}}=: e_{\mathrm{t} 2}
$$

In order to establish limit theory for the kernel estimator in (2.1), we use the following regularity conditions.

Assumption 1. Let $e_{\mathrm{t}}=\left(e_{\mathrm{t} 0}, e_{\mathrm{t} 1}^{\prime}, e_{\mathrm{t} 2}^{\prime}\right)^{\prime}$ satisfy

$$
e_{t}=\sum_{j=0}^{\infty} \Phi_{j} \varepsilon_{t-j}=\sum_{j=0}^{\infty} \Phi_{j} L^{j} \varepsilon_{t}=: \Phi(L) \varepsilon_{t},
$$

where $L$ is the lag operator, $\left\{\boldsymbol{\Phi}_{j}\right\}$ is a sequence of $(d+1) \times(d+1)$ matrices and $\left\{\varepsilon_{t}\right\}$ is a sequence of i.i.d. $(\mathrm{d}+1)$-dimensional random vectors with mean zero, $\Omega_{\varepsilon}:=\mathrm{E}\left[\varepsilon_{\mathrm{t}} \varepsilon_{\mathrm{t}}^{\prime}\right]$ being positive definite and $\mathrm{E}\left[\left\|\varepsilon_{\mathrm{t}}\right\|^{4+\delta_{0}}\right]<\infty$ for $\delta_{0}>0$. In addition, the multivariate linear process coefficient matrices satisfy $\sum_{j=0}^{\infty} j\left\|\Phi_{j}\right\|<\infty$ and the matrix $\Omega:=\Phi \Omega_{\varepsilon} \Phi^{\prime}$ is positive definite with $\Phi:=\sum_{j=0}^{\infty} \Phi_{j} \neq$ $\mathbf{O}_{(d+1) \times(d+1)}$.

ASSUMPTION 2. The $\mathrm{d}$-dimensional coefficient function $\boldsymbol{\beta}(\cdot)$ is continuous with $\|\boldsymbol{\beta}(z+s)-\boldsymbol{\beta}(z)\|=\mathrm{O}\left(|\mathbf{s}|^{\delta_{1}}\right)$ as $s \rightarrow 0$ for any $0<z<1$, where $\delta_{1}$ is a positive constant satisfying $\frac{1}{2}<\delta_{1} \leqslant 1$.

ASSUMPTION 3. (i) The kernel function $\mathrm{K}(\cdot)$ is continuous, positive, symmetric and has compact support $[-1,1]$ with $\mu_{0}=\int \mathrm{K}(\mathrm{u}) \mathrm{du}=1$.

(ii) The bandwidth $\mathrm{h}$ satisfies $\mathrm{h} \rightarrow 0$ and $\mathrm{Th} \rightarrow \infty$ as $\mathrm{T} \rightarrow \infty$.

Assumption 1 uses a stationary vector linear process specification for $\left\{e_{t}\right\}$ that is common in the literature (c.f., Phillips, 1995; Phillips, Li and Gao, 2017) and includes many popular vector time series processes such as stationary VAR and VARMA models (Lütkepohl, 2006). The linear process dependence structure can be replaced by alternative mixing dependence conditions with some modifications of the proofs. Assumption 1 
combined with (2.2) implies that the nonstationary components $\mathbf{H}_{2}^{\prime} X_{t}$ are full rank nonstationary and not cointegrated. In the asymptotic theory developed later, $\mathbf{H}_{2}^{\prime} X_{t}$ may be correlated with $e_{t 0}$, which implies endogeneity in the system. Assumptions 2 and 3 are commonly used conditions in the varying-coefficient and kernel smoothing literature - e.g., Wang and Phillips (2009b) and Phillips, Li and Gao (2017). In particular, if the coefficient function $\beta(\cdot)$ is Lipschitz continuous on $[0,1]$, Assumption 2 is satisfied with $\delta_{1}=1$. When greater smoothness conditions are imposed on $\beta(\cdot)$ stronger results are possible with local linear and polynomial smoothing methods, and these will be mentioned in what follows.

\subsection{Kernel degeneracy and double-rotation of the covariate space}

By virtue of Assumption 1, Beveridge-Nelson decomposition and functional limit theory for linear processes (e.g., Theorem 3.4(b) in Phillips and Solo, 1992),

$$
\mathrm{T}^{-1 / 2} \sum_{\mathrm{t}=1}^{\lfloor\mathrm{T} z\rfloor} e_{\mathrm{t}}=\mathrm{T}^{-1 / 2} \sum_{\mathrm{t}=1}^{\lfloor\mathrm{T} z\rfloor} \Phi \varepsilon_{\mathrm{t}}+\mathrm{o}_{\mathrm{P}}(1) \Rightarrow \mathrm{B}(z), 0<z \leqslant 1
$$

where $e_{\mathrm{t}}=\left(e_{\mathrm{t} 0}, e_{\mathrm{t} 1}^{\prime}, e_{\mathrm{t} 2}^{\prime}\right)^{\prime}, \mathrm{B}(z)$ is a $(\mathrm{d}+1)$-dimensional Brownian motion with variance matrix $\Omega=\boldsymbol{\Phi} \boldsymbol{\Omega}_{\varepsilon} \boldsymbol{\Phi}^{\prime}$ defined in Assumption 1 , and $\lfloor\cdot\rfloor$ denotes the floor function. Partition the $(d+1) \times(d+1)$ matrix $\Omega$ into cell submatrices $\Omega_{i j}(i, j=0,1,2)$ conformably with $e_{t}$ and set $\omega=\Omega_{00}$. Let $B(z)=\left[B_{0}(z), B_{1}^{\prime}(z), B_{2}^{\prime}(z)\right]^{\prime}$ be the Brownian motion limit process of the following component partial sum processes

$$
T^{-1 / 2} \sum_{t=1}^{\lfloor T z\rfloor} e_{t j} \Rightarrow B_{j}(z), j=0,1,2 \text {, }
$$

where $\mathrm{B}_{0}(\cdot), \mathrm{B}_{1}(\cdot)$ and $\mathrm{B}_{2}(\cdot)$ are univariate, $\mathrm{d}_{0}$-dimensional and $\mathrm{d}_{1}$-dimensional Brownian motions with variance matrices $\omega, \Omega_{11}$ and $\Omega_{22}$, respectively. The limit theory later in the paper also involves partitioned components of the one-sided long run covariance matrix defined by $\Gamma:=\operatorname{lrcov}^{+}\left(e_{t}, e_{t}\right)=\sum_{h=0}^{\infty} E\left[e_{0} e_{h}^{\prime}\right]$ with cell submatrices $\Gamma_{i j}(i, j=0,1,2)$ that are conformable with the partition of $e_{t}$.

When the $p$-dimensional process $\left\{Z_{t}\right\}$ is stationary and satisfies some standard regularity conditions, it is not difficult to show that

$$
\frac{1}{T h} \sum_{t=1}^{T} Z_{t} Z_{t}^{\prime} K\left(\frac{t-T z_{0}}{T h}\right)=\mu_{0} E\left[Z_{t} Z_{t}^{\prime}\right]+o_{P}(1)=E\left[Z_{t} Z_{t}^{\prime}\right]+o_{P}(1),
$$

where $\mu_{0}=\int K(u) d u=1$. Furthermore, if $E\left[Z_{t} Z_{t}^{\prime}\right]$ is positive definite, the limit of the inverse of the kernel weighted sample moment matrix $\frac{1}{T h} \sum_{t=1}^{T} Z_{t} Z_{t}^{\prime} K\left(\frac{t-T z_{0}}{T h}\right)$ also exists and conventional asymptotics hold for kernel estimation with $Z_{t}$ as regressors. However, when $\left\{Z_{t}\right\}$ is generated by a nonstationary full rank unit root process with innovations that satisfy a functional law similar to (2.4), the weighted sample moment matrix behaves very differently. First, we have $T^{-1 / 2} Z_{\lfloor T z\rfloor} \Rightarrow B_{\diamond}(z)$ for $0<z \leqslant 1$, where $B_{\diamond}(\cdot)$ is a p-dimensional Brownian motion with positive definite variance matrix, from which it might be expected that the normalization rate $(T h)$ in (2.6) would simply be replaced by the rate $\left(T^{2} h\right)$. However, Phillips, Li and Gao (2017) showed that the matrix $\frac{1}{T^{2} h} \sum_{t=1}^{T} Z_{t} Z_{t}^{\prime} K\left(\frac{t-T z_{0}}{T h}\right)$ is asymptotically singular when the dimension $p$ exceeds unity. The reason for this degeneracy is that time-varying coefficient kernel regression concentrates attention in the nonstationary process on a particular time coordinate (say $\mathrm{T} z_{0}$ ) and the corresponding 
realized value of the associated limit of the nonstationary process, in contrast to the time average $E\left[Z_{t} Z_{t}^{\prime}\right]$ in stationary case. When there are multiple nonstationary regressors, this focus on a single time coordinate produces a limiting signal matrix of deficient rank unity whose zero eigenspace depends on the value of the limit process at that time coordinate. In other words, the kernel induced degeneracy which occurs in the matrix $\frac{1}{T^{2} h} \sum_{t=1}^{T} Z_{t} Z_{t}^{\prime} K\left(\frac{t-T z_{0}}{T h}\right)$ for multivariate integrated $Z_{t}$ is random, trajectory dependent, and localized to the time value $z_{0}$. It may therefore be regarded as a form of local degeneracy.

To deal with degeneracy in a prototypical case, Phillips, Li and Gao (2017) introduced a novel rotational decomposition for the kernel-weighted signal matrix $\sum_{t=1}^{T} Z_{t} Z_{t}^{\prime} K\left(\frac{t-T z_{0}}{T h}\right)$ to develop the limit theory. The rotation involved the use of a random direction based on the nonstationary regressors. In the present case, this direction takes the form of the (sample size dependent) vector

$$
\mathrm{q}_{\mathrm{T}}\left(z_{0}\right)=\frac{\mathrm{b}_{\mathrm{T}}\left(z_{0}\right)}{\left[\mathrm{b}_{\mathrm{T}}\left(z_{0}\right)^{\prime} \mathrm{b}_{\mathrm{T}}\left(z_{0}\right)\right]^{1 / 2}}=\frac{\mathrm{b}_{\mathrm{T}}\left(z_{0}\right)}{\left\|\mathrm{b}_{\mathrm{T}}\left(z_{0}\right)\right\|}, \quad \mathrm{b}_{\mathrm{T}}\left(z_{0}\right)=\mathrm{T}^{-1 / 2} \mathrm{Z}_{\delta\left(z_{0}\right)}, \delta\left(z_{0}\right)=\left\lfloor\mathrm{T}\left(z_{0}-\mathrm{h}\right)\right\rfloor,
$$

leading to an associated orthogonal matrix

$$
\mathbf{Q}_{\mathrm{T}}\left(z_{0}\right)=\left[\mathbf{q}_{\mathrm{T}}\left(z_{0}\right), \mathbf{q}_{\mathrm{T}}^{\perp}\left(z_{0}\right)\right], \mathbf{Q}_{\mathrm{T}}\left(z_{0}\right)^{\prime} \mathbf{Q}_{\mathrm{T}}\left(z_{0}\right)=\mathbf{I}_{\mathfrak{p}},
$$

where $\mathrm{q}_{\mathrm{T}}^{\perp}\left(z_{0}\right)$ is an orthogonal complement to $\mathrm{q}_{\mathrm{T}}\left(z_{0}\right)$ whose existence is not unique (say, $-\mathrm{q}_{\mathrm{T}}^{\perp}\left(z_{0}\right)$ is also an orthogonal complement to $\left.\mathrm{q}_{\mathrm{T}}\left(z_{0}\right)\right)$. Using the standardization matrix

$$
\mathbf{D}_{\mathrm{T}}=\operatorname{diag}\left\{\mathrm{T} \sqrt{\mathrm{h}},(\mathrm{Th}) \mathbf{I}_{\mathrm{p}-1}\right\}
$$

and Proposition A.1 from Phillips, Li and Gao (2017), we may show that the matrix

$$
\mathbf{D}_{\mathrm{T}}^{-1} \mathbf{Q}_{\mathrm{T}}\left(z_{0}\right)^{\prime}\left[\sum_{\mathrm{t}=1}^{\mathrm{T}} \mathrm{Z}_{\mathrm{t}} \mathbf{Z}_{\mathrm{t}}^{\prime} \mathrm{K}\left(\frac{\mathrm{t}-\mathrm{T} z_{0}}{\mathrm{Th}}\right)\right] \mathbf{Q}_{\mathrm{T}}\left(z_{0}\right) \mathbf{D}_{\mathrm{T}}^{-1}
$$

is of full rank with probability approaching one.

This random rotation technique needs substantial generalization for the setting in the present paper. Here the regressors satisfy the framework (2.2), indicating that three different normalization rates might be needed when $d_{0} \geqslant 1$ and $d_{1} \geqslant 2$, where $d_{0}$ and $d_{1}$ are the dimensions of the stationary components and nonstationary components. To see this, we first use the orthogonal transformation (2.2) to rotate the regressor space and separate out the stationary and nonstationary components. Define $X_{t 1}=H_{1}^{\prime} X_{t}, X_{t 2}=H_{2}^{\prime} X_{t}$, where $X_{t 1}$ is the $d_{0}$-dimensional stationary component and $X_{t 2}$ is the $d_{1}$-dimensional nonstationary component with unit roots. Then, model (1.1) can be re-written as

$$
Y_{\mathrm{t}}=\boldsymbol{\beta}_{\mathrm{t} 1}^{\prime} X_{\mathrm{t} 1}+\boldsymbol{\beta}_{\mathrm{t} 2}^{\prime} X_{\mathrm{t} 2}+e_{\mathrm{t} 0}
$$

with $\boldsymbol{\beta}_{\mathrm{t} 1}^{\prime}=\boldsymbol{\beta}_{\mathrm{t}}^{\prime} \mathbf{H}_{1}$ and $\boldsymbol{\beta}_{\mathrm{t} 2}^{\prime}=\boldsymbol{\beta}_{\mathrm{t}}^{\prime} \mathbf{H}_{2}$. Letting $\bar{X}_{\mathrm{t}}=\left(X_{\mathrm{t} 1}^{\prime}, X_{\mathrm{t} 2}^{\prime}\right)^{\prime}=\mathbf{H}^{\prime} \mathbf{X}_{\mathrm{t}}$, we transform the Nadaraya-Watson kernel estimate $\widehat{\boldsymbol{\beta}}\left(z_{0}\right)$ to

$$
\overline{\boldsymbol{\beta}}\left(z_{0}\right):=\mathbf{H}^{\prime} \widehat{\boldsymbol{\beta}}\left(z_{0}\right)=\left[\sum_{t=1}^{\mathrm{T}} \bar{X}_{\mathrm{t}} \bar{X}_{\mathrm{t}}^{\prime} \mathrm{K}\left(\frac{\mathrm{t}-\mathrm{T} z_{0}}{\mathrm{Th}}\right)\right]^{+}\left[\sum_{\mathrm{t}=1}^{\mathrm{T}} \overline{\mathrm{X}}_{\mathrm{t}} \mathrm{Y}_{\mathrm{t}} \mathrm{K}\left(\frac{\mathrm{t}-\mathrm{T} z_{0}}{\mathrm{Th}}\right)\right],
$$


which is the estimate of $\mathbf{H}^{\prime} \boldsymbol{\beta}\left(z_{0}\right)$. The component matrix $\mathbf{H}_{1}$ generates the stationary components and the convergence rate in this direction will be seen to be the same as the usual convergence rate in stationary kernel regression. In contrast, the component matrix $\mathbf{H}_{2}$, which is orthogonal to $\mathbf{H}_{1}$, generates full rank nonstationary variates, leading to faster convergence rates in this direction. However, the above arguments show that the matrix $\frac{1}{T^{2} h} \sum_{t=1}^{T} X_{t 2} X_{t 2}^{\prime} K\left(\frac{t-T z_{0}}{T h}\right)$ is asymptotically singular if its dimension $d_{1}$ exceeds unity. Therefore, further transformation of the nonstationary component $X_{t 2}$ is required in order to resolve asymptotic behavior.

To proceed, let $\mathrm{q}_{\mathrm{T} 2}\left(z_{0}\right)$ and $\mathrm{Q}_{\mathrm{T} 2}\left(z_{0}\right)$ be defined just as $\mathrm{q}_{\mathrm{T}}\left(z_{0}\right)$ and $\mathrm{Q}_{\mathrm{T}}\left(z_{0}\right)$ above but with $Z_{\mathrm{t}}$ replaced by $X_{\mathrm{t} 2}$. Then define

$$
\overline{\mathbf{Q}}_{\mathrm{T}}\left(z_{0}\right)=\operatorname{diag}\left\{\mathbf{I}_{\mathrm{d}_{0}}, \mathbf{Q}_{\mathrm{T} 2}\left(z_{0}\right)\right\}, \overline{\mathbf{D}}_{\mathrm{T}}=\operatorname{diag}\left\{\sqrt{\mathrm{Th}} \mathbf{I}_{\mathrm{d}_{0}}, \mathrm{~T} \sqrt{\mathrm{h}},(\mathrm{Th}) \mathbf{I}_{\mathrm{d}_{1}-1}\right\}
$$

Unlike the transformation matrix $\mathbf{H}$ in the global rotation which does not rely on $z_{0}$, the matrix $\overline{\mathbf{Q}}_{\mathrm{T}}\left(z_{0}\right)$ used in the further rotation of the nonstationary component space is random and time dependent on $z_{0}$, and is thus called a local rotation. Proposition 1 below shows that the matrix $\overline{\mathbf{D}}_{\mathrm{T}}^{-1} \overline{\mathbf{Q}}_{\mathrm{T}}\left(z_{0}\right)^{\prime} \mathbf{H}^{\prime} \boldsymbol{\Lambda}_{\mathrm{T}}\left(z_{0}\right) \mathbf{H} \overline{\mathbf{Q}}_{\mathrm{T}}\left(z_{0}\right) \overline{\mathbf{D}}_{\mathrm{T}}^{-1}$ is of full rank with probability approaching one as $T \rightarrow \infty$, where $\Lambda_{\mathrm{T}}\left(z_{0}\right)$ is defined in (2.1).

To complete the statement of the proposition we introduce the following notation. Define the vector

$$
\mathrm{q}_{2}\left(z_{0}\right)=\frac{\mathrm{b}\left(z_{0}\right)}{\left[\mathrm{b}\left(z_{0}\right)^{\prime} \mathrm{b}\left(z_{0}\right)\right]^{1 / 2}}=\frac{\mathrm{b}\left(z_{0}\right)}{\left\|\mathrm{b}\left(z_{0}\right)\right\|} \text { with } \mathrm{b}\left(z_{0}\right)=\mathrm{B}_{2}\left(z_{0}\right)
$$

and let $\mathrm{q}_{2}^{\perp}\left(z_{0}\right)$ be a $\mathrm{d}_{1} \times\left(\mathrm{d}_{1}-1\right)$ orthogonal complement matrix of $\mathrm{q}_{2}\left(z_{0}\right)$ and the limit of $\mathrm{q}_{\mathrm{T} 2}^{\perp}\left(z_{0}\right)$. Define the $d \times d$ matrix

$$
\boldsymbol{\Lambda}\left(z_{0}\right)=\operatorname{diag}\left\{\boldsymbol{\Lambda}_{11}, \boldsymbol{\Lambda}_{2}\left(z_{0}\right)\right\} \text { with } \boldsymbol{\Lambda}_{2}\left(z_{0}\right)=\left[\begin{array}{ll}
\Lambda_{22}\left(z_{0}\right) & \Lambda_{23}\left(z_{0}\right) \\
\Lambda_{32}\left(z_{0}\right) & \Lambda_{33}\left(z_{0}\right)
\end{array}\right]
$$

where $\Lambda_{11}=\mathrm{E}\left[\mathrm{e}_{11} e_{11}^{\prime}\right]>0$ is independent of $z_{0}, \boldsymbol{\Lambda}_{22}\left(z_{0}\right)=\lambda\left(z_{0}\right)=\mathrm{B}_{2}\left(z_{0}\right)^{\prime} \mathrm{B}_{2}\left(z_{0}\right)$ is univariate,

$$
\begin{aligned}
& \Lambda_{23}\left(z_{0}\right)=\Lambda_{32}\left(z_{0}\right)^{\prime}=\sqrt{2}\left[\mathrm{~B}_{2}\left(z_{0}\right)^{\prime} \mathrm{B}_{2}\left(z_{0}\right)\right]^{1 / 2}\left[\int_{-1}^{1} \mathrm{~B}_{2}^{*}\left(\frac{z+1}{2}\right)^{\prime} \mathrm{K}(z) \mathrm{d} z\right] \mathrm{q}_{2}^{\perp}\left(z_{0}\right), \\
& \Lambda_{33}\left(z_{0}\right)=2 \mathrm{q}_{2}^{\perp}\left(z_{0}\right)^{\prime}\left[\int_{-1}^{1} \mathrm{~B}_{2}^{*}\left(\frac{z+1}{2}\right) \mathrm{B}_{2}^{*}\left(\frac{z+1}{2}\right)^{\prime} \mathrm{K}(z) \mathrm{d} z\right] \mathrm{q}_{2}^{\perp}\left(z_{0}\right),
\end{aligned}
$$

and $\mathrm{B}_{2}^{*}(\cdot)$ is an independent copy of the Brownian motion $\mathrm{B}_{2}(\cdot)$. In the sequel, "> 0 " denotes positive definiteness of the relevant matrix.

Proposition 1. Suppose that Assumptions 1 and 3 are satisfied, $\mathrm{d} \geqslant 3$ with $1 \leqslant \mathrm{~d}_{0} \leqslant \mathrm{~d}-2$ and $2 \leqslant \mathrm{~d}_{1} \leqslant \mathrm{~d}-\mathrm{d}_{0}$. Then we have

$$
\begin{aligned}
\overline{\mathbf{D}}_{\mathrm{T}}^{-1} \overline{\mathbf{Q}}_{\mathrm{T}}\left(z_{0}\right)^{\prime} \mathbf{H}^{\prime} \boldsymbol{\Lambda}_{\mathrm{T}}\left(z_{0}\right) \mathbf{H} \overline{\mathbf{Q}}_{\mathrm{T}}\left(z_{0}\right) \overline{\mathbf{D}}_{\mathrm{T}}^{-1} & =\overline{\mathbf{D}}_{\mathrm{T}}^{-1} \overline{\mathbf{Q}}_{\mathrm{T}}\left(z_{0}\right)^{\prime}\left[\sum_{\mathrm{t}=1}^{\mathrm{T}} \overline{\mathrm{X}}_{\mathrm{t}} \overline{\mathrm{X}}_{\mathrm{t}}^{\prime} \mathrm{K}\left(\frac{\mathrm{t}-\mathrm{T} z_{0}}{\mathrm{Th}}\right)\right] \overline{\mathbf{Q}}_{\mathrm{T}}\left(z_{0}\right) \overline{\mathbf{D}}_{\mathrm{T}}^{-1} \\
& \Rightarrow \boldsymbol{\Lambda}\left(z_{0}\right)>0 \text { a.s. }
\end{aligned}
$$

for fixed $0<z_{0}<1$. 
REMARK 1. This proposition resolves the asymptotic degeneracy of the kernel-weighted signal matrix through a double-rotation of the nonstationary regressor space involving the global rotation $\mathrm{H}$ and local rotation $\overline{\mathbf{Q}}_{\mathrm{T}}\left(z_{0}\right)$. This transformation leads to three different normalization rates embodied in the standardization matrix $\overline{\mathbf{D}}_{\mathrm{T}}$. For a special case $\mathrm{d}_{1}=1$, kernel degeneracy is circumvented and the rate $(\mathrm{Th})$ disappears in $\overline{\mathbf{D}}_{\mathrm{T}}$, leaving only the global rotation $\mathrm{H}$. If there is no cointegration among the regressors, the global rotation is not needed in transforming the regressors and the rate $(\sqrt{\mathrm{Th}})$ would disappear in $\overline{\mathbf{D}}_{\mathrm{T}}$, specializing the result to Proposition A.1 in Phillips, Li and Gao (2017).

\subsection{Asymptotic theory for cointegrated regressors}

This section derives asymptotic theory for the kernel estimator $\widehat{\boldsymbol{\beta}}\left(z_{0}\right)$ when the nonstationary regressors are cointegrated. We start by introducing notation. Let $\overline{\mathbf{D}}_{\mathrm{T} 2}=\operatorname{diag}\left\{\mathrm{T} \sqrt{\mathrm{h}},(\mathrm{Th}) \mathbf{I}_{\mathrm{d}_{1}-1}\right\}$ and define $\Delta_{2}\left(z_{0}\right):=$ $\left[\bar{\delta}\left(z_{0}\right), \Delta_{\perp}\left(z_{0}\right)^{\prime}\right]^{\prime}$ with

$$
\begin{aligned}
& \bar{\delta}\left(z_{0}\right):=\left[2 \mathrm{~B}_{2}\left(z_{0}\right)^{\prime} \mathrm{B}_{2}\left(z_{0}\right)\right]^{1 / 2} \int_{-1}^{1} \mathrm{~K}(z) \mathrm{dB}_{0}\left(\frac{z+1}{2}\right), \\
& \Delta_{\perp}\left(z_{0}\right):=2 \mathrm{q}_{2}^{\perp}\left(z_{0}\right)^{\prime}\left[\int_{-1}^{1} \mathrm{~K}(z) \mathrm{B}_{2}^{*}\left(\frac{z+1}{2}\right) \mathrm{dB} \mathrm{B}_{0}\left(\frac{z+1}{2}\right)+\frac{1}{2} \Gamma_{20}\right],
\end{aligned}
$$

where the one sided long run covariance $\Gamma_{20}=\operatorname{lrcov}^{+}\left(e_{t 2}, e_{t 0}\right)$ is as defined earlier. The following theorem gives the asymptotic distribution of $\widehat{\boldsymbol{\beta}}\left(z_{0}\right)$.

Theorem 1. Suppose Assumptions 1-3 are satisfied, $d \geqslant 3$ with $1 \leqslant d_{0} \leqslant d-2$ and $2 \leqslant d_{1} \leqslant d-d_{0}, \Lambda\left(z_{0}\right)$ is non-singular with probability one, and for $\mathrm{s} \geqslant \mathrm{t}, \mathrm{E}\left[e_{\mathrm{s} 0} e_{\mathrm{t} 1}\right]=\mathbf{0}_{\mathrm{d}_{0}}$, where $\mathbf{0}_{\mathrm{r}}$ is an $\mathrm{r}$-dimensional vector of zeros.

(i) If, in addition, $\mathrm{Th}^{1+2 \delta_{1}}=\mathrm{o}(1)$, then as $\mathrm{T} \rightarrow \infty$

$$
\sqrt{\operatorname{Th}} \mathbf{H}_{1}^{\prime}\left[\widehat{\boldsymbol{\beta}}\left(z_{0}\right)-\boldsymbol{\beta}\left(z_{0}\right)\right] \Rightarrow \xi
$$

for fixed $z_{0} \in(0,1)$, where $\xi$ is a $\mathrm{d}_{0}$-dimensional normal vector with mean zero and covariance matrix

$$
\mathbf{V}_{\xi}:=v_{0} \Lambda_{11}^{+}\left\{\sum_{s=-\infty}^{\infty} E\left[\left(e_{10} e_{s 0}\right)\left(e_{11} e_{s 1}^{\prime}\right)\right]\right\} \Lambda_{11}^{+} \text {, with } v_{0}=\int K^{2}(u) d u
$$

(ii) If, in addition, $\mathrm{T}^{2} \mathrm{~h}^{1+2 \delta_{1}}=\mathrm{o}(1)$, then as $\mathrm{T} \rightarrow \infty$

$$
\overline{\mathbf{D}}_{\mathrm{T} 2} \overline{\mathbf{Q}}_{\mathrm{T} 2}\left(z_{0}\right)^{\prime} \mathbf{H}_{2}^{\prime}\left[\widehat{\boldsymbol{\beta}}\left(z_{0}\right)-\boldsymbol{\beta}\left(z_{0}\right)\right] \Rightarrow \boldsymbol{\Lambda}_{2}^{-1}\left(z_{0}\right) \boldsymbol{\Delta}_{2}\left(z_{0}\right)
$$

for fixed $z_{0} \in(0,1)$.

REMARK 2. (a) The limit theory in Theorem 1 shows that double-rotation of the regressor space is needed to characterize the asymptotics: the global rotator $\mathbf{H}$ addresses potential cointegration among the nonstationary regressors; and the local rotator $\overline{\mathbf{Q}}_{\mathrm{T}}\left(z_{0}\right)$ addresses the kernel degeneracy that arises from the fixed design functional framework. The limit theory in (2.12) and (2.13) encompasses several interesting results from the existing literature. For the case $d_{1}=0$ corresponding to a stationary regressor model, taking $\mathbf{H}_{1}=\mathbf{I}_{d}$ we 
have from (2.12) that

$$
\sqrt{\mathrm{Th}}\left[\widehat{\boldsymbol{\beta}}\left(z_{0}\right)-\boldsymbol{\beta}\left(z_{0}\right)\right] \Rightarrow \xi,
$$

which delivers results similar to those in the literature on kernel estimation with locally stationary regressors (c.f., Robinson, 1989; Cai, 2007; Vogt, 2012). For the case $d_{0}=0$ corresponding to a full rank integrated regressor model, taking $\mathbf{H}_{2}=\mathbf{I}_{\mathbf{d}}$ we obtain Theorem 3.1 in Phillips, Li and Gao (2017) as a corollary of (2.13).

(b) The assumption $E\left[e_{s 0} e_{t 1}\right]=0$ for $s \geqslant t$ implies no contemporaneous or feedforward correlation between the stationary regressor components $e_{t 1}$ and the equation errors $e_{t 0}$, which ensures kernel estimation is consistent in the direction associated with the stationary components $\mathbf{H}_{1}^{\prime} \boldsymbol{X}_{\mathbf{t}}$ (c.f., Park and Phillips, 1989). Theorem 1 does not specify the relationship between the limit distributions of the stationary and nonstationary component estimators in parts (i) and (ii) and to do so we impose the following explicit exogeneity condition.

ASSUMPTION $1^{*}$. Let $\mathcal{F}_{\mathrm{t}-1}=\sigma\left(e_{\mathrm{t} 1}, e_{\mathrm{t} 2}, e_{\mathrm{t}-1}, e_{\mathrm{t}-2}, \cdots\right)$ be the $\sigma$-algebra generated by $\left\{e_{\mathrm{s} 1}, e_{\mathrm{s} 2}\right\}_{\mathrm{s} \leqslant \mathrm{t}}$ and $\left\{e_{s 0}\right\}_{s \leqslant t-1}$. Then $\left\{\left(e_{\mathrm{t} 0}, \mathcal{F}_{\mathrm{t}}\right)\right\}$ is a stationary sequence of martingale differences with $\sigma_{e}^{2}=\mathrm{E}\left[e_{\mathrm{t} 0}^{2} \mid \mathcal{F}_{\mathrm{t}-1}\right]>0$ a.s.

Under Assumption 1*, the asymptotic distribution in the direction $\mathbf{H}_{1}$ is independent of that in the direction $\mathbf{H}_{2}$, so that the limit variate $\xi$ is independent of the limit variate $\Lambda_{2}^{+}\left(z_{0}\right) \Delta_{2}\left(z_{0}\right)$, which facilitates inference concerning the time varying coefficient function. Further, the one-sided long run covariance matrix $\Gamma_{02}$ is eliminated in the random variate $\Delta_{2}\left(z_{0}\right)$ for this pure cointegration case. Assumption $1^{*}$ is common in the literature when stationarity is present and appears, for instance, in both Cai, Li and Park (2009) and Li, Phillips and Gao (2016).

(c) From (2.12) and (2.13) in Theorem 1, we find three different convergence rates that apply in different directions. In the direction $\mathbf{H}_{1}$, by (2.12) we have the well-known stationary rate given by

$$
\mathbf{H}_{1}^{\prime}\left[\widehat{\boldsymbol{\beta}}\left(z_{0}\right)-\boldsymbol{\beta}\left(z_{0}\right)\right]=\mathrm{O}_{\mathrm{P}}\left(\frac{1}{\sqrt{\mathrm{Th}}}\right),
$$

which holds for stationary kernel regression. In the direction $\mathrm{H}_{2} \mathrm{q}_{\mathrm{T} 2}\left(z_{0}\right)$, we have the faster rate

$$
\mathrm{q}_{\mathrm{T} 2}\left(z_{0}\right)^{\prime} \mathbf{H}_{2}^{\prime}\left[\widehat{\boldsymbol{\beta}}\left(z_{0}\right)-\boldsymbol{\beta}\left(z_{0}\right)\right]=\mathrm{O}_{\mathrm{P}}\left(\frac{1}{\mathrm{~T} \sqrt{\mathrm{h}}}\right),
$$

which is called type 1 super-consistency in Li, Phillips and Gao (2016) and Phillips, Li and Gao (2017). Finally, in direction $\mathrm{H}_{2} \mathrm{q}_{\mathrm{T} 2}^{\perp}\left(z_{0}\right)$, we have type 2 super-consistency with rate given by

$$
\mathrm{q}_{\mathrm{T} 2}^{\perp}\left(z_{0}\right)^{\prime} \mathbf{H}_{2}^{\prime}\left[\widehat{\boldsymbol{\beta}}\left(z_{0}\right)-\boldsymbol{\beta}\left(z_{0}\right)\right]=\mathrm{O}_{\mathrm{P}}\left(\frac{1}{\mathrm{Th}}\right) .
$$

The type 2 super-consistency rate is slower than the rate in (2.16), but is still faster than the stationary rate in (2.15). Interestingly, therefore, nonstationary regressors raise the rate of convergence over the standard stationary rate in the two relevant directions of nonstationarity in the data.

(d) The bandwidth conditions $\mathrm{Th}^{1+2 \gamma}=\mathrm{o}(1)$ and $\mathrm{T}^{2} \mathrm{~h}^{1+2 \gamma}=\mathrm{o}(1)$ in Theorem 1 may appear restrictive. However, if the coefficient function has continuous derivatives up to the second order and if we apply local linear kernel smoothing rather than local constant estimation, then following the proof of Theorem 3.2 in Phillips, Li and Gao (2017), we may relax the above two bandwidth restrictions to $\mathrm{Th}^{5}=\mathrm{o}(1)$ and $\mathrm{T}^{2} \mathrm{~h}^{5}=\mathrm{o}(1)$, respectively (c.f., Remark 4 in Section 3.1). 
(e) Theorem 1 implies that $\widehat{\boldsymbol{\beta}}\left(z_{0}\right)$ has a degenerate asymptotic normal distribution dominated by the slowest convergent component in (2.12), viz.,

$$
\begin{aligned}
\sqrt{\operatorname{Th}}\left[\widehat{\boldsymbol{\beta}}\left(z_{0}\right)-\boldsymbol{\beta}\left(z_{0}\right)\right] & =\sqrt{\operatorname{Th}} \mathbf{H}^{\prime}\left[\widehat{\boldsymbol{\beta}}\left(z_{0}\right)-\boldsymbol{\beta}\left(z_{0}\right)\right] \\
& =\sqrt{\operatorname{Th}} \mathbf{H}_{1} \mathbf{H}_{1}^{\prime}\left[\widehat{\boldsymbol{\beta}}\left(z_{0}\right)-\boldsymbol{\beta}\left(z_{0}\right)\right]+\sqrt{\operatorname{Th}} \mathbf{H}_{2} \mathbf{H}_{2}^{\prime}\left[\widehat{\boldsymbol{\beta}}\left(z_{0}\right)-\boldsymbol{\beta}\left(z_{0}\right)\right] \\
& \Rightarrow \mathbf{H}_{1} \xi+\mathrm{O}_{\mathrm{P}}(1 / \sqrt{\mathrm{Th}}) \\
& =\mathbf{H}_{1} \xi+\mathrm{o}_{\mathrm{P}}(1) .
\end{aligned}
$$

This also shows that the kernel estimate $\widehat{\boldsymbol{\beta}}\left(z_{0}\right)$ is consistent with the stationary pointwise convergence rate $(\sqrt{\mathrm{Th}})$ as long as $d_{0} \geqslant 1$. In spite of this apparent simplification arising from the dominating direction $\mathbf{H}_{1}$, inference about the full vector of parameters $\beta$ is typically not degenerate and involves the asymptotic behavior of the components of $\beta$ in other directions. On the other hand, if $d>1$ but $d_{0}=0$, corresponding to the full rank integrated regressor setting as in Phillips, Li and Gao (2017), we may show that

$$
\begin{aligned}
\widehat{\boldsymbol{\beta}}\left(z_{0}\right)-\boldsymbol{\beta}\left(z_{0}\right) & =\overline{\mathbf{Q}}_{\mathrm{T} 2}\left(z_{0}\right) \overline{\mathbf{Q}}_{\mathrm{T} 2}\left(z_{0}\right)^{\prime}\left[\widehat{\boldsymbol{\beta}}\left(z_{0}\right)-\boldsymbol{\beta}\left(z_{0}\right)\right] \\
& =\mathrm{q}_{\mathrm{T} 2}\left(z_{0}\right) \mathrm{q}_{\mathrm{T} 2}\left(z_{0}\right)^{\prime}\left[\widehat{\boldsymbol{\beta}}\left(z_{0}\right)-\boldsymbol{\beta}\left(z_{0}\right)\right]+\mathrm{q}_{\mathrm{T} 2}^{\perp}\left(z_{0}\right) \mathrm{q}_{\mathrm{T} 2}^{\perp}\left(z_{0}\right)^{\prime}\left[\widehat{\boldsymbol{\beta}}\left(z_{0}\right)-\boldsymbol{\beta}\left(z_{0}\right)\right],
\end{aligned}
$$

where $\mathrm{q}_{\mathrm{T} 2}\left(z_{0}\right), \mathrm{q}_{\mathrm{T} 2}^{\perp}\left(z_{0}\right)$ and $\overline{\mathbf{Q}}_{\mathrm{T} 2}\left(z_{0}\right)$ are defined as those in Theorem 1(ii) but with $\mathrm{d}_{1}$ and $\mathrm{X}_{\mathrm{t} 2}$ replaced by $\mathrm{d}$ and $X_{t}$, respectively. Taking $H_{2}=I_{d}$ in (2.16) and (2.17) and noting that $\mathrm{q}_{\mathrm{T} 2}\left(z_{0}\right)$ and $\mathrm{q}_{\mathrm{T} 2}^{\perp}\left(z_{0}\right)$ are both $\mathrm{O}_{\mathrm{P}}(1)$, we can prove that $\widehat{\boldsymbol{\beta}}\left(z_{0}\right)$ is consistent with the type 2 super-consistency rate $(\mathrm{Th})$.

(f) The above kernel regression asymptotics are applicable to the multiple time-varying coefficient regression model with intercept, i.e.,

$$
Y_{t}=\beta_{t 0}+\beta_{t}^{\prime} X_{t}+e_{t 0}, t=1, \cdots, T,
$$

where $\beta_{0 t}:=\beta_{0}(t / T)$ is a continuous time-varying intercept function and the remaining elements satisfy the corresponding conditions imposed for model (1.1). Under (2.19), we only need to slightly modify the global rotation, replacing the matrix $\mathrm{H}$ by

$$
\overline{\mathbf{H}}=\operatorname{diag}\{1, \mathbf{H}\}=\left(\overline{\mathbf{H}}_{1}, \overline{\mathbf{H}}_{2}\right)
$$

with

$$
\overline{\mathbf{H}}_{1}=\left(\begin{array}{cc}
1 & \mathbf{0}_{\mathrm{d}_{0}}^{\prime} \\
\mathbf{0}_{\mathrm{d}} & \mathbf{H}_{1}
\end{array}\right), \overline{\mathbf{H}}_{2}=\left(\begin{array}{c}
\mathbf{0}_{\mathrm{d}_{1}}^{\prime} \\
\mathbf{H}_{2}
\end{array}\right) .
$$

Applying $\overline{\mathbf{H}}_{1}$ and $\overline{\mathbf{H}}_{2}$ on the $(\mathrm{d}+1)$-dimensional regressor vector $\left(1, X_{t}^{\prime}\right)^{\prime}$, we may separate out the stationary and nonstationary components. The implementation of local rotation remains the same by noting that $\overline{\mathbf{H}}_{2}^{\prime}\left(1, X_{\mathrm{t}}^{\prime}\right)^{\prime}=\mathbf{H}_{2}^{\prime} X_{\mathrm{t}}$.

\section{Extensions of the kernel estimation theory}

This section develops kernel estimation theory for the following two cases: (i) the regressors $X_{t}$ have a mixture of deterministic and stochastic trends but no internal cointegrating structure; and (ii) the regressors $X_{t}$ have deterministic trends and are cointegrated among themselves. 


\subsection{Kernel estimation with stochastic and deterministic trends}

We assume the regressors are generated as stochastic trends with drift according to the scheme

$$
X_{t}=X_{t-1}+\mu+u_{t}
$$

where $\boldsymbol{\mu}$ is a $\mathrm{d}$-dimensional parameter vector representing the accompanying drift of the unit root process, and where $u_{\mathrm{t}}=\left(e_{\mathrm{t} 1}^{\prime}, e_{\mathrm{t} 2}^{\prime}\right)^{\prime}$ with $e_{\mathrm{t} 1}$ and $e_{\mathrm{t} 2}$ satisfying Assumption 1 in Section 2.1. From (3.1), we have

$$
\begin{aligned}
X_{t} & =\sum_{j=1}^{t} u_{j}+\mu t+X_{0} \\
& =: S_{t}+D_{t}+X_{0}
\end{aligned}
$$

where $X_{0}=O_{P}(1), S_{t}:=\sum_{j=1}^{t} u_{j}$ is the stochastic trend and $D_{t}:=\mu t$ is the deterministic drift. Although we consider only a linear trend for $D_{t}$ in what follows, the method and theory developed in this section are readily extendable to polynomial trends. But general power trends such as $t^{\alpha}$ with unknown power parameter $\alpha$ involve further complications of asymptotic singularity - see Phillips (2007) and Baek, Cho and Phillips (2015), which are not pursued here.

Since $S_{t}=O_{P}\left(t^{1 / 2}\right)$, the stochastic trend $S_{t}$ is asymptotically dominated by the deterministic trend $D_{t}$. Therefore, we have

$$
\sum_{t=1}^{T} X_{t} X_{t}^{\prime} K\left(\frac{t-T z_{0}}{T h}\right)=\mu \mu^{\prime}\left(\left\lfloor T z_{0}\right\rfloor\right)^{2} T h\left(1+o_{P}(1)\right) .
$$

When the dimension d exceeds unity, the matrix $\mu \mu^{\prime}$ is singular, complicating normalization of the kernelweighted signal matrix on the right hand side of (3.3), which is degenerate at the dominating rate $\left(T^{3} h\right)$ associated with the deterministic component $D_{t}$. Degeneracy of this form has long been studied in the linear nonstationary regression literature, where Park and Phillips (1988) gave a global rotation technique (with non-random transformation matrix) to separate out the stochastic and deterministic trend components with associated standardization rates for the corresponding directions. This global rotation technique cannot be applied in the present setting, however, as will be demonstrated later in this section. Since the kernel-weighted signal matrix embodies both stochastic and deterministic trends, a local rotation technique similar to that in Section 2.2 is instead required.

To proceed, define

$$
\widetilde{\mathrm{q}}_{\mathrm{T}}\left(z_{0}\right)=\frac{\widetilde{\mathrm{b}}_{\mathrm{T}}\left(z_{0}\right)}{\left[\widetilde{\mathrm{b}}_{\mathrm{T}}\left(z_{0}\right)^{\prime} \widetilde{\mathrm{b}}_{\mathrm{T}}\left(z_{0}\right)\right]^{1 / 2}}=\frac{\widetilde{\mathrm{b}}_{\mathrm{T}}\left(z_{0}\right)}{\left\|\widetilde{\mathrm{b}}_{\mathrm{T}}\left(z_{0}\right)\right\|}, \quad \widetilde{\mathrm{b}}_{\mathrm{T}}\left(z_{0}\right)=\mathrm{X}_{\delta\left(z_{0}\right)}=\mathrm{S}_{\delta\left(z_{0}\right)}+\mathrm{D}_{\delta\left(z_{0}\right)}+\mathrm{X}_{0},
$$

where $\delta\left(z_{0}\right)=\left\lfloor T\left(z_{0}-h\right)\right\rfloor$, and introduce the orthogonal matrix

$$
\widetilde{\mathbf{Q}}_{\mathrm{T}}\left(z_{0}\right)=\left[\widetilde{\mathrm{q}}_{\mathrm{T}}\left(z_{0}\right), \widetilde{\mathbf{q}}_{\mathrm{T}}^{\perp}\left(z_{0}\right)\right], \widetilde{\mathbf{Q}}_{\mathrm{T}}\left(z_{0}\right)^{\prime} \widetilde{\mathbf{Q}}_{\mathrm{T}}\left(z_{0}\right)=\mathbf{I}_{\mathrm{d}},
$$

where $\widetilde{\mathbf{q}}_{\mathrm{T}}^{\perp}\left(z_{0}\right)$ is a $\mathrm{d} \times(\mathrm{d}-1)$ orthogonal complement matrix of $\widetilde{\mathbf{q}}_{\mathrm{T}}\left(z_{0}\right)$, and define the standardization matrix

$$
\widetilde{\mathbf{D}}_{\mathrm{T}}=\operatorname{diag}\left\{\mathrm{T} \sqrt{\mathrm{Th}},(\mathrm{Th}) \mathbf{I}_{\mathrm{d}-1}\right\}
$$


Proposition 2 below shows that the asymptotic kernel degeneracy is addressed via application of the local rotator and path-dependent transformation matrix $\widetilde{\mathbf{Q}}_{\mathrm{T}}\left(z_{0}\right)$ defined in (3.4). Some further notation is needed to state the proposition. Let $\widetilde{\boldsymbol{\mu}}_{\perp}$ be a $\mathrm{d} \times(\mathrm{d}-1)$ orthogonal complement of $\tilde{\boldsymbol{\mu}}:=\left(\boldsymbol{\mu}^{\prime} \boldsymbol{\mu}\right)^{-1 / 2} \boldsymbol{\mu}=\boldsymbol{\mu} /\|\boldsymbol{\mu}\|$, and $\mathbf{J}=\left(\widetilde{\boldsymbol{\mu}}, \tilde{\boldsymbol{\mu}}_{\perp}\right)$ be the corresponding orthogonal transformation matrix, as used in Park and Phillips (1988). Define the $d \times d$ matrix

$$
\tilde{\Lambda}\left(z_{0}\right)=\left[\begin{array}{cc}
\tilde{\Lambda}_{11}\left(z_{0}\right) & \tilde{\Lambda}_{12}\left(z_{0}\right) \\
\tilde{\Lambda}_{21}\left(z_{0}\right) & \widetilde{\Lambda}_{22}
\end{array}\right]
$$

where $\tilde{\boldsymbol{\Lambda}}_{11}\left(z_{0}\right)=\widetilde{\lambda}\left(z_{0}\right)=\left\|\boldsymbol{\mu} z_{0}\right\|^{2}$ is non-random and univariate,

$$
\begin{aligned}
& \tilde{\boldsymbol{\Lambda}}_{12}\left(z_{0}\right)=\widetilde{\boldsymbol{\Lambda}}_{21}\left(z_{0}\right)^{\prime}=\sqrt{2}\left\|\boldsymbol{\mu} z_{0}\right\|\left[\int_{-1}^{1} \widetilde{\mathrm{B}}\left(\frac{z+1}{2}\right)^{\prime} \mathrm{K}(z) \mathrm{d} z\right] \widetilde{\boldsymbol{\mu}}_{\perp} \\
& \tilde{\boldsymbol{\Lambda}}_{22}=2 \widetilde{\boldsymbol{\mu}}_{\perp}^{\prime}\left[\int_{-1}^{1} \widetilde{\mathrm{B}}\left(\frac{z+1}{2}\right) \widetilde{\mathrm{B}}\left(\frac{z+1}{2}\right)^{\prime} \mathrm{K}(z) \mathrm{d} z\right] \widetilde{\boldsymbol{\mu}}_{\perp}
\end{aligned}
$$

$\widetilde{\mathrm{B}}(\cdot)=\left[\mathrm{B}_{1}(\cdot)^{\prime}, \mathrm{B}_{2}(\cdot)^{\prime}\right]^{\prime}, \mathrm{B}_{1}(\cdot)$ and $\mathrm{B}_{2}(\cdot)$ are defined earlier in $(2.5)$.

Proposition 2. Suppose Assumptions 1 and 3 are satisfied, $\boldsymbol{\mu} \neq \mathbf{0}_{\mathrm{d}}$ and $\mathrm{d} \geqslant 2$. Then

$$
\widetilde{\mathbf{D}}_{\mathrm{T}}^{-1} \widetilde{\mathbf{Q}}_{\mathrm{T}}\left(z_{0}\right)^{\prime} \boldsymbol{\Lambda}_{\mathrm{T}}\left(z_{0}\right) \widetilde{\mathbf{Q}}_{\mathrm{T}}\left(z_{0}\right) \widetilde{\mathbf{D}}_{\mathrm{T}}^{-1} \Rightarrow \widetilde{\boldsymbol{\Lambda}}\left(z_{0}\right)>0 \text { a.s. }
$$

for fixed $0<z_{0}<1$.

REMARK 3. In the proof of (3.6) in Appendix A, the two random and trajectory-dependent directions $\widetilde{\mathrm{q}}_{\mathrm{T}}\left(z_{0}\right)$ and $\widetilde{\mathbf{q}}_{\mathrm{T}}^{\perp}\left(z_{0}\right)$ are shown to converge to $\widetilde{\boldsymbol{\mu}}$ and $\widetilde{\boldsymbol{\mu}}_{\perp}$, respectively. Both $\widetilde{\boldsymbol{\mu}}$ and $\widetilde{\boldsymbol{\mu}}_{\perp}$ are non-random, and neither of them rely on $z_{0}$. This is unsurprising as the nonstationary process $X_{t}$ is asymptotically dominated by its linear trend $D_{t}$. A natural question in view of this asymptotic behavior is whether the local transformation matrix $\widetilde{\mathbf{Q}}_{\boldsymbol{\top}}\left(z_{0}\right)$ can be replaced by the global matrix $\mathbf{J}=\left(\widetilde{\boldsymbol{\mu}}, \widetilde{\boldsymbol{\mu}}_{\perp}\right)$ in (3.6)? The question is simply answered by examining the special case $d=2$. Define

$$
\widetilde{X}_{\mathrm{t}}:=\mathrm{J}^{\prime} \mathrm{X}_{\mathrm{t}}=\left(\widetilde{\boldsymbol{\mu}}^{\prime} \mathrm{X}_{\mathrm{t}}, \widetilde{\boldsymbol{\mu}}_{\perp}^{\prime} \mathrm{X}_{\mathrm{t}}\right)^{\prime}=:\left(\widetilde{\mathrm{X}}_{\mathrm{t} 1}, \widetilde{\mathrm{X}}_{\mathrm{t} 2}\right)^{\prime}
$$

It is easy to see that the univariate component $\widetilde{X}_{t 1}$ represents the deterministic trend term, whereas $\widetilde{X}_{\mathrm{t} 2}$ represents a unit root process without the involvement of the deterministic trend. Defining $\widetilde{\mathbf{D}}_{\mathrm{T} *}=$ $\operatorname{diag}\{T \sqrt{T h}, T \sqrt{h}\}$, we can show that

$$
\widetilde{\mathbf{D}}_{\mathrm{T} *}^{-1}\left[\sum_{t=1}^{\mathrm{T}} \widetilde{\mathrm{X}}_{\mathrm{t}} \widetilde{\mathrm{X}}_{\mathrm{t}}^{\prime} \mathrm{K}\left(\frac{\mathrm{t}-\mathrm{T} z_{0}}{\mathrm{Th}}\right)\right] \widetilde{\mathbf{D}}_{\mathrm{T} *}^{-1} \Rightarrow\left[\begin{array}{cc}
\left\|\boldsymbol{\mu} z_{0}\right\|^{2} & \left\|\boldsymbol{\mu} z_{0}\right\|\left\|\widetilde{\boldsymbol{\mu}}_{\perp}^{\prime} \widetilde{\mathrm{B}}\left(z_{0}\right)\right\| \\
\left\|\boldsymbol{\mu} z_{0}\right\|\left\|\widetilde{\boldsymbol{\mu}}_{\perp}^{\prime} \widetilde{\mathrm{B}}\left(z_{0}\right)\right\| & \left\|\widetilde{\boldsymbol{\mu}}_{\perp}^{\prime} \widetilde{\mathrm{B}}\left(z_{0}\right)\right\|^{2}
\end{array}\right] .
$$

The above result is easily established by noting that the asymptotic leading terms for $\widetilde{X}_{t 1}$ and $\widetilde{X}_{t 2}$ are $\left\|\mu z_{0}\right\| \cdot T$ and $S_{\delta\left(z_{0}\right)}$, respectively, when $T\left(z_{0}-h\right) \leqslant t \leqslant T\left(z_{0}+h\right)$. Clearly, the matrix on the right side of (3.8) is singular with probability one. This outcome shows that use of the global (limit) transformation matrix $\mathbf{J}=\left(\widetilde{\boldsymbol{\mu}}, \widetilde{\boldsymbol{\mu}}_{\perp}\right)$ inadequately deals with the kernel signal matrix degeneracy even though the two relevant directions $\widetilde{\mu}$ and $\widetilde{\boldsymbol{\mu}}_{\perp}$ figure prominently in the limit. Instead, the local rotator $\widetilde{\mathbf{Q}}_{\mathrm{T}}\left(z_{0}\right)$ and the associated normalization matrix $\widetilde{\mathbf{D}}_{\mathrm{T}}=\operatorname{diag}\left\{\mathrm{T} \sqrt{\mathrm{Th}},(\mathrm{Th}) \mathbf{I}_{\mathrm{d}-1}\right\}$ in place of $\widetilde{\mathbf{D}}_{\mathrm{T} * \text { play key roles in achieving }}$ 
a non-degenerate limit theory.

With Proposition 2 in hand, the limit theory for the kernel estimator $\widehat{\boldsymbol{\beta}}\left(z_{0}\right)$ can now be obtained for stochastic trend with drift regressors, as in (3.1). Let

$$
\widetilde{\Delta}\left(z_{0}\right)=\left[\widetilde{\delta}\left(z_{0}\right), \widetilde{\Delta}_{\perp}^{\prime}\right]^{\prime}
$$

with

$$
\begin{aligned}
\widetilde{\delta}\left(z_{0}\right) & =\sqrt{2}\left\|\mu z_{0}\right\| \int_{-1}^{1} \mathrm{~K}(z) \mathrm{dB}_{0}\left(\frac{z+1}{2}\right), \\
\widetilde{\Delta}_{\perp} & =2 \widetilde{\mu}_{\perp}^{\prime}\left[\int_{-1}^{1} \mathrm{~K}(z) \widetilde{\mathrm{B}}\left(\frac{z+1}{2}\right) \mathrm{dB}_{0}\left(\frac{z+1}{2}\right)+\frac{1}{2} \widetilde{\Gamma}\right],
\end{aligned}
$$

where $\widetilde{\Gamma}=\left(\Gamma_{10}^{\prime}, \Gamma_{20}^{\prime}\right)^{\prime}$, and the one-sided long run covariance matrices $\Gamma_{10}=\operatorname{lrcov}^{+}\left(e_{\mathrm{t} 1}, e_{\mathrm{t} 0}\right), \Gamma_{20}=$ $\operatorname{lrcov}^{+}\left(e_{\mathrm{t} 2}, e_{\mathrm{t} 0}\right)$ are defined in Section 2.2. The following theorem gives the asymptotic distribution of $\widehat{\boldsymbol{\beta}}\left(z_{0}\right)$.

THEOREM 2. Suppose Assumptions $1-3$ are satisfied, $\boldsymbol{\mu} \neq \mathbf{0}_{\mathrm{d}}$, and $\mathrm{d} \geqslant 2$. Then, as $\mathrm{T} \rightarrow \infty$, we have

$$
\widetilde{\mathbf{D}}_{\mathrm{T}} \widetilde{\mathbf{Q}}_{\mathrm{T}}\left(z_{0}\right)^{\prime}\left[\widehat{\boldsymbol{\beta}}\left(z_{0}\right)-\boldsymbol{\beta}\left(z_{0}\right)+\mathrm{O}_{\mathrm{P}}\left(\mathrm{h}^{\delta_{1}}\right)\right] \Rightarrow \widetilde{\boldsymbol{\Lambda}}^{-1}\left(z_{0}\right) \tilde{\boldsymbol{\Delta}}\left(z_{0}\right),
$$

for fixed $z_{0} \in(0,1)$.

REMARK 4. (a) Since the limit of the direction $\widetilde{\mathrm{q}}_{\mathrm{T}}^{\perp}\left(z_{0}\right)$ is independent of $z_{0}$, it is interesting to find that both the random matrix $\widetilde{\Lambda}_{22}$ (the lower-right block matrix of $\widetilde{\Lambda}\left(z_{0}\right)$ ) and the random vector $\widetilde{\Delta}_{\perp}$ are also independent of $z_{0}$. In the above theorem, in order to make the bias term asymptotically negligible, we have to impose the strong restriction $\mathrm{T}^{3} \mathrm{~h}^{1+2 \delta_{1}}=\mathrm{o}(1)$, which contradicts the normal bandwidth condition Th $\rightarrow \infty$ made in Assumption 3(ii) since $\delta_{1} \in(0.5,1]$. However, as discussed in Remark 2(d), if the timevarying coefficient functions have continuous derivatives up to the second order, we can use the local linear smoothing estimation and consequently relax the bandwidth restriction. For fixed $0<z_{0}<1$, the following local linear approximation holds when $z$ is in a small neighborhood of $z_{0}$ :

$$
\boldsymbol{\beta}(z) \approx \beta\left(z_{0}\right)+\boldsymbol{\beta}^{(1)}\left(z_{0}\right)\left(z-z_{0}\right),
$$

where $\boldsymbol{\beta}^{(1)}\left(z_{0}\right)$ is the first-order derivative of $\beta(\cdot)$ at $z_{0}$. Define the local linear loss function

$$
\mathcal{L}_{\mathrm{T}}(\mathbf{a}, \mathbf{b})=\sum_{\mathrm{t}=1}^{\mathrm{T}}\left[\mathrm{Y}_{\mathrm{t}}-\mathrm{X}_{\mathrm{t}}^{\prime} \mathbf{a}-\mathrm{X}_{\mathrm{t}}^{\prime} \mathbf{b}\left(\frac{\mathrm{t}}{\mathrm{T}}-z_{0}\right)\right]^{2} \mathrm{~K}\left(\frac{\mathrm{t}-\mathrm{T} z_{0}}{\mathrm{Th}}\right),
$$

where $\boldsymbol{a}=\left(a_{1}, \cdots, a_{d}\right)^{\prime}$ and $\mathbf{b}=\left(b_{1}, \cdots, b_{d}\right)^{\prime}$. Then, the local linear estimator of $\beta\left(z_{0}\right)$ is defined as $\widetilde{\boldsymbol{\beta}}\left(z_{0}\right)=\widetilde{\mathbf{a}}$, where

$$
(\widetilde{\mathbf{a}}, \widetilde{\mathbf{b}})=\arg \min _{(\mathbf{a}, \mathbf{b})} \mathcal{L}_{\mathrm{T}}(\mathbf{a}, \mathbf{b}) .
$$

With some standard arguments, the asymptotic bias term of the local linear estimator has the order of $\mathrm{O}\left(\mathrm{h}^{2}\right)$, leading to the replacement of the bandwidth requirement $T^{3} h^{1+2 \delta_{1}}=o(1)$ in Theorem 2 by the weaker condition $\mathrm{T}^{3} \mathrm{~h}^{5}=\mathrm{o}(1)$, which is compatible with $\mathrm{Th} \rightarrow \infty$.

(b) Compared with Theorem 1 in Section 2.3, there is a single rotator matrix $\widetilde{\mathbf{Q}}_{\mathrm{T}}\left(z_{0}\right)$ involved in the limit 
theory. In consequence, we have two different convergence rates. In the direction $\widetilde{\mathrm{q}}_{\mathrm{T}}\left(z_{0}\right)$, we have

$$
\widetilde{\mathrm{q}}_{\mathrm{T}}\left(z_{0}\right)^{\prime}\left[\widehat{\boldsymbol{\beta}}\left(z_{0}\right)-\boldsymbol{\beta}\left(z_{0}\right)\right]=\mathrm{O}_{\mathrm{P}}\left(\frac{1}{\mathrm{~T} \sqrt{\mathrm{Th}}}+\mathrm{h}^{\delta_{1}}\right)
$$

where $1 /(T \sqrt{T h})$ is the new super-consistency convergence rate for nonstationary kernel regression that exceeds the rates in (2.15)-(2.17). This fast rate is mainly due to the strong signal from the linear trend of $X_{t}$ in the direction $\widetilde{\mathbf{q}}_{\mathrm{T}}\left(z_{0}\right)$. In contrast, in the direction of $\widetilde{\mathbf{q}}_{\mathrm{T}}^{\perp}\left(z_{0}\right)$, from (3.9), we have

$$
\widetilde{\mathrm{q}}_{\mathrm{T}}^{\perp}\left(z_{0}\right)^{\prime}\left[\widehat{\boldsymbol{\beta}}\left(z_{0}\right)-\boldsymbol{\beta}\left(z_{0}\right)\right]=\mathrm{O}_{\mathrm{P}}\left(\frac{1}{\mathrm{Th}}+\mathrm{h}^{\delta_{1}}\right),
$$

which is the same rate as that for type 2 super-consistency in (2.17) if the bias term order is ignored asymptotically. This rate is due to the relatively weaker signal that emerges in the direction $\widetilde{\mathrm{q}}_{\mathrm{T}}^{\perp}\left(z_{0}\right)$ as the linear trend cancels out through the transform $\widetilde{\mathrm{q}}_{\mathrm{T}}^{\perp}\left(z_{0}\right)$.

\subsection{Kernel cointegrating regression with deterministic trends}

Next, we combine the structures (2.2) and (3.1) and assume that $X_{t}$ satisfies

$$
\mathbf{H}_{1}^{\prime} X_{\mathrm{t}}=e_{\mathrm{t} 1}, \Delta\left(\mathbf{H}_{2}^{\prime} X_{\mathrm{t}}\right)=\mathbf{H}_{2}^{\prime}\left(\Delta \mathrm{X}_{\mathrm{t}}\right)=e_{\mathrm{t} 2}+\boldsymbol{\mu},
$$

where $\mathbf{H}_{1}, \mathbf{H}_{2}, e_{\mathrm{t} 1}$ and $e_{\mathrm{t} 2}$ are defined as in Section 2.1 and $\boldsymbol{\mu}$ is defined as in Section 3.1 (with the dimension $\mathrm{d}$ replaced by $d_{1}=d-d_{0}$ ). The following example shows that the structure (3.13) is satisfied for a cointegrated VAR model with a deterministic drift component.

EXAMPLE 2. Consider the VAR model defined by

$$
\Delta X_{\mathrm{t}}=\alpha \gamma^{\prime} \mathrm{X}_{\mathrm{t}-1}+v+v_{\mathrm{t}}
$$

where $\left\{v_{\mathrm{t}}\right\}$ is a covariance stationary sequence of random vectors with mean zero, $\alpha$ and $\gamma$ are $d \times d_{0}$ constant matrices of rank $d_{0}$, and $v$ is a d-dimensional parameter vector. Letting $\gamma_{\perp}$ and $\alpha_{\perp}$ be $d \times\left(d-d_{0}\right)$ matrices of full rank satisfying $\gamma^{\prime} \gamma_{\perp}=\mathrm{O}_{\mathrm{d}_{0} \times \mathrm{d}_{1}}$ and $\boldsymbol{\alpha}^{\prime} \boldsymbol{\alpha}_{\perp}=\mathrm{O}_{\mathrm{d}_{0} \times \mathrm{d}_{1}}$, and defining $\mathrm{C}=\gamma_{\perp}\left(\boldsymbol{\alpha}_{\perp}^{\prime} \gamma_{\perp}\right)^{-1} \boldsymbol{\alpha}_{\perp}^{\prime}$, we have the Granger representation

$$
\mathrm{X}_{\mathrm{t}}=\mathrm{C} \sum_{j=1}^{\mathrm{t}} v_{j}+\mathrm{C} v \mathrm{t}+\mathrm{C} \mathrm{X}_{0}+\mathrm{v}_{\mathrm{t}}
$$

where $V_{t}=\alpha\left(\gamma^{\prime} \boldsymbol{\alpha}\right)^{-1} \sum_{i=0}^{\infty} R^{i} \gamma^{\prime} v_{t-i}$ is a stationary linear process and the matrix $R=I_{d_{0}}+\gamma^{\prime} \boldsymbol{\alpha}$ has eigenvalues within the unit circle (Johansen, 1991; Cheng and Phillips, 2009). By choosing $\mathbf{H}_{1}$ and $\mathbf{H}_{2}$ as in Example 1, it is clear that the cointegrated VAR model (3.14) satisfies the structure (3.13).

To derive the limit theory of $\widehat{\boldsymbol{\beta}}\left(z_{0}\right)$ under the generating mechanism (3.13) for $X_{t}$, we first apply the transformation matrix $\mathbf{H}=\left(\mathbf{H}_{1}, \mathbf{H}_{2}\right)$ on the covariate space as in Section 2.2 to separate out stationary and nonstationary components as $X_{t 1}=H_{1}^{\prime} X_{t}$ and $X_{t 2}=H_{2}^{\prime} X_{t}$. For the nonstationary elements $X_{t 2}$, a further local rotation using the matrix $\overline{\mathbf{Q}}_{\mathrm{T}}\left(z_{0}\right)$ defined in (2.9) is applied to overcome kernel degeneracy. Proposition 3 below shows that this double-rotation technique leads to a well defined limit theory for the kernel-weighted signal matrix when $X_{t}$ is generated by (3.13). 
Define the standardization matrix

$$
\stackrel{\circ}{\mathbf{D}}_{\mathrm{T}}=\operatorname{diag}\left\{\sqrt{\mathrm{Th}} \mathbf{I}_{\mathrm{d}_{0}}, \mathrm{~T} \sqrt{\mathrm{Th}},(\mathrm{Th}) \mathbf{I}_{\mathrm{d}_{1}-1}\right\}
$$

and the $d \times d$ matrix

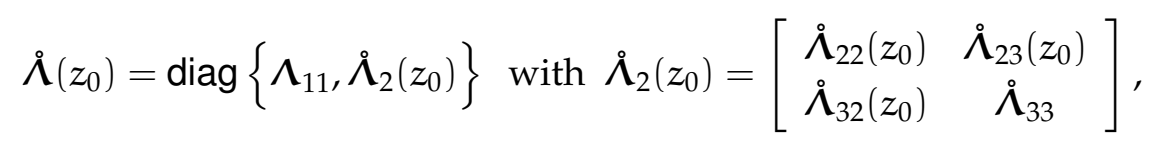

where $\boldsymbol{\Lambda}_{11}$ is defined as in Section 2.2, $\stackrel{\Lambda}{22}_{2}\left(z_{0}\right)=\grave{\lambda}\left(z_{0}\right)=\left\|\boldsymbol{\mu} z_{0}\right\|^{2}$ is non-random and univariate,

$$
\begin{aligned}
& \stackrel{\Lambda}{23}_{2}\left(z_{0}\right)=\stackrel{\Lambda}{32}_{3}\left(z_{0}\right)^{\prime}=\sqrt{2}\left\|\boldsymbol{\mu} z_{0}\right\|\left[\int_{-1}^{1} \mathrm{~B}_{2}\left(\frac{z+1}{2}\right)^{\prime} \mathrm{K}(z) \mathrm{d} z\right] \tilde{\boldsymbol{\mu}}_{\perp}, \\
& \stackrel{\Lambda}{33}_{2}=2 \widetilde{\boldsymbol{\mu}}_{\perp}^{\prime}\left[\int_{-1}^{1} \mathrm{~B}_{2}\left(\frac{z+1}{2}\right) \mathrm{B}_{2}\left(\frac{z+1}{2}\right)^{\prime} \mathrm{K}(z) \mathrm{d} z\right] \tilde{\boldsymbol{\mu}}_{\perp},
\end{aligned}
$$

$\mathrm{B}_{2}(\cdot)$ is defined in (2.5) and $\widetilde{\boldsymbol{\mu}}_{\perp}$ is defined as in Section 3.1.

Proposition 3. Suppose Assumptions 1 and 3 are satisfied, $\boldsymbol{\mu} \neq \mathbf{0}_{\mathrm{d}}, \mathrm{d} \geqslant 3$ with $1 \leqslant \mathrm{~d}_{0} \leqslant \mathrm{~d}-2$ and $2 \leqslant d_{1} \leqslant d-d_{0}$. Then

$$
\stackrel{\circ}{\mathbf{D}}_{\mathrm{T}}^{-1} \overline{\mathbf{Q}}_{\mathrm{T}}\left(z_{0}\right)^{\prime} \mathbf{H}^{\prime} \Lambda_{\mathrm{T}}\left(z_{0}\right) \mathbf{H} \overline{\mathbf{Q}}_{\mathrm{T}}\left(z_{0}\right) \stackrel{\circ}{\mathbf{D}}_{\mathrm{T}}^{-1} \Rightarrow \stackrel{\wedge}{\Lambda}\left(z_{0}\right)>0 \text { a.s. }
$$

for fixed $0<z_{0}<1$.

REMARK 5. The limit result is similar to that in Proposition 1, with two differences. First, the normalization rate $(T \sqrt{h})$ in $\overline{\mathbf{D}}_{\mathrm{T}}$ is replaced by the rate $(T \sqrt{T h})$ in $\stackrel{\circ}{\mathrm{D}}_{\mathrm{T}}$, which is due to the fact that the stochastic trend is asymptotically dominated by the linear trend in the direction of $\mathbf{H}_{2}$. Second, the limits of the directions $\mathrm{q}_{\mathrm{T} 2}\left(z_{0}\right)$ and $\mathrm{q}_{\mathrm{T} 2}^{\perp}\left(z_{0}\right)$ in the above proposition are non-random and independent of the point $z_{0}$ (i.e., $\tilde{\boldsymbol{\mu}}$ and $\tilde{\boldsymbol{\mu}}_{\perp}$ ), whereas the corresponding limits in Proposition 1 are random and depend on $z_{0}$.

To provide the limit distribution of the kernel estimator under (3.13), we introduce further notation, defining $\stackrel{\circ}{\mathrm{T} 2}=\operatorname{diag}\left\{\mathrm{T} \sqrt{\mathrm{Th}},(\mathrm{Th}) \mathbf{I}_{\mathrm{d}_{1}-1}\right\}$ and $\stackrel{\circ}{2}_{2}\left(z_{0}\right)=\left[\stackrel{\circ}{\delta}\left(z_{0}\right), \stackrel{\Delta}{\Delta}_{\perp}^{\prime}\right]^{\prime}$, with

$$
\begin{aligned}
& \stackrel{\delta}{\delta}\left(z_{0}\right)=\sqrt{2}\left\|\mu z_{0}\right\| \int_{-1}^{1} \mathrm{~K}(z) \mathrm{dB}_{0}\left(\frac{z+1}{2}\right), \\
& \AA_{\perp}=2 \widetilde{\boldsymbol{\mu}}_{\perp}^{\prime}\left[\int_{-1}^{1} \mathrm{~K}(z) \mathrm{B}_{2}\left(\frac{z+1}{2}\right) \mathrm{dB}_{0}\left(\frac{z+1}{2}\right)+\frac{1}{2} \Gamma_{20}\right] .
\end{aligned}
$$

The limit theory for $\widehat{\boldsymbol{\beta}}\left(z_{0}\right)$ is as follows.

THEOREM 3. Suppose that Assumptions 1-3 are satisfied, $\boldsymbol{\mu} \neq \mathbf{0}_{\mathrm{d}}, \mathrm{d} \geqslant 3$ with $1 \leqslant \mathrm{~d}_{0} \leqslant \mathrm{~d}-2$ and $2 \leqslant \mathrm{~d}_{1} \leqslant \mathrm{~d}-\mathrm{d}_{0}$, and $\stackrel{\Upsilon}{\Lambda}\left(z_{0}\right)$ is non-singular with probability one. For $\mathrm{s} \geqslant \mathrm{t}, \mathrm{E}\left[e_{\mathrm{s} 0} e_{\mathrm{t} 1}\right]=\mathbf{0}_{\mathrm{d}_{0}}$.

(i) If, in addition, $\mathrm{Th}^{1+2 \delta_{1}}=\mathrm{o}(1)$, then (2.12) holds as $\mathrm{T} \rightarrow \infty$.

(ii) For fixed $z_{0} \in(0,1)$,

$$
\stackrel{\circ}{\mathrm{T} 2}_{\mathbf{Q}_{\mathrm{T} 2}}\left(z_{0}\right)^{\prime} \mathbf{H}_{2}^{\prime}\left[\widehat{\boldsymbol{\beta}}\left(z_{0}\right)-\boldsymbol{\beta}\left(z_{0}\right)+\mathrm{O}_{\mathrm{P}}\left(\mathrm{h}^{\delta_{1}}\right)\right] \Rightarrow \stackrel{\Lambda}{2}_{2}^{-1}\left(z_{0}\right) \check{\Delta}_{2}\left(z_{0}\right)
$$


as $\mathrm{T} \rightarrow \infty$

REMARK 6. Theorem 3 combines parts of Theorems 1 and 2, showing that application of the rotation matrices $\mathbf{H}$ and $\overline{\mathbf{Q}}_{\mathrm{T}}\left(z_{0}\right)$ resolves the degeneracy issue in kernel-weighted signal matrices in a similar way to the transformations in Section 2. However, due to the presence of deterministic trends, the standardization matrix needs modification and leads to a faster convergence rate in the direction $H_{2} q_{T 2}\left(z_{0}\right)$, viz.,

$$
\mathrm{q}_{\mathrm{T} 2}\left(z_{0}\right)^{\prime} \mathbf{H}_{2}^{\prime}\left[\widehat{\boldsymbol{\beta}}\left(z_{0}\right)-\boldsymbol{\beta}\left(z_{0}\right)\right]=\mathrm{O}_{\mathrm{P}}\left(\frac{1}{\mathrm{~T} \sqrt{\mathrm{Th}}}+\mathrm{h}^{\delta_{1}}\right)
$$

analogous to that in (3.9). Furthermore, as noted in Remark 2(b), under the additional Assumption $1^{*}$, the limit distributions in Theorem 3(i) and (ii) are mutually independent.

\section{FM-kernel estimation}

As is apparent in the limit distributions obtained earlier, second-order bias effects are present in the asymptotics whenever the regressors are endogenous. Just as in linear cointegration regression asymptotics, endogeneity may be addressed by using modified estimation methods, such as those in Phillips and Hansen (1990). This section provides a kernel modification of the Phillips-Hansen approach that is called FM-kernel estimation. This method effectively removes second-order bias effects in the limit distribution associated with the nonstationary direction $\mathbf{H}_{2}$. To save space, we mainly focus on the case of cointegrated regressors studied in Section 2, and the development of FM-kernel regression is entirely analogous for the cases of mixed stochastic and deterministic trend regressors and cointegrated regressors with deterministic trends considered in Section 3. Methods other than FM-kernel regression may also be designed to resolve endogeneity and serial correlation induced bias issues, just as they are in linear cointegrated regression models. But these are not pursued here. From Theorem 1(ii), the presence of the one-sided long run covariance $\Gamma_{20}$ between $e_{\mathrm{t} 0}$ and $e_{\mathrm{t} 2}$, induces a second-order bias effect in the limit distribution in the direction $\mathbf{H}_{2} q_{\mathrm{T} 2}^{\perp}\left(z_{0}\right)$. In addition, there is endogeneity arising from correlation between the limit Brownian motions $\mathrm{B}_{0}(\cdot)$ and $\mathrm{B}_{2}(\cdot)$. These bias effects relate directly to those that are present in linear cointegrating regression limit theory as discussed originally in Park and Phillips (1988, 1989) and Phillips and Hansen (1990). Although the existence of this bias does not affect the super-consistency rates of kernel estimation, centering is affected, with consequential impact on statistical inference concerning the coefficient functions. The need to remove these sources of bias and to provide valid inferential machinery motivates the development of an FM-kernel estimator.

FM least squares estimation was introduced by Phillips and Hansen (1990) in the context of traditional linear cointegrating models, and was recently generalized by Phillips, Li and Gao (2017) to nonparametric kernel-based estimation in models with full rank integrated regressors. When nonstationary regressors are cointegrated, they are necessarily of deficient rank asymptotically, therefore complicating the development of FM-kernel estimation methodology. To present the required modifications more clearly, we start the development under the assumption (which is later relaxed) that the cointegration rank $\mathrm{d}_{0}$ and the transformation matrix $H$ are known a priori, together with the long-run covariance matrices $\Omega_{02}, \Omega_{20}, \Omega_{22}, \Gamma_{20}$ and $\Gamma_{22}$. 
Correction for endogeneity is achieved by removing the following term in kernel estimation

$$
\mathbf{B}_{\mathrm{T} 1}^{\star}\left(z_{0}\right)=\sum_{\mathrm{t}=1}^{\mathrm{T}} \mathrm{K}\left(\frac{\mathrm{t}-\mathrm{T} z_{0}}{\mathrm{Th}}\right) \mathrm{X}_{\mathrm{t}} \odot\left[\mathbf{0}_{\mathrm{d}_{0}}^{\prime}\left(\boldsymbol{\Omega}_{02} \boldsymbol{\Omega}_{22}^{+} \Delta \mathrm{X}_{\mathrm{t} 2} \mathbf{1}_{\mathrm{d}_{1}}\right)^{\prime}\right]^{\prime},
$$

where $\mathbf{1}_{d_{1}}$ is a $d_{1}$-dimensional column vector of ones and $\odot$ denotes component-wise product. Correction for serial correlation is achieved by removing the term

$$
\mathbf{B}_{\mathrm{T} 2}^{\star}\left(z_{0}\right)=\mathbf{H} \overline{\mathbf{Q}}_{\mathrm{T}}\left(z_{0}\right) \overline{\mathbf{D}}_{\mathrm{T}}\left\{\mathbf{0}_{\mathrm{d}_{0}+1}^{\prime},\left[\mathrm{q}_{\mathrm{T} 2}^{\perp}\left(z_{0}\right)^{\prime}\left(\Gamma_{20}-\Gamma_{22} \Omega_{22}^{+} \Omega_{20}\right)\right]^{\prime}\right\}^{\prime} .
$$

Combining (4.1) and (4.2), the (infeasible) FM-kernel estimator is constructed as

$$
\widehat{\boldsymbol{\beta}}_{\star}\left(z_{0}\right)=\boldsymbol{\Lambda}_{\mathrm{T}}^{+}\left(z_{0}\right) \Delta_{\mathrm{T}}^{\star}\left(z_{0}\right) \text {, with } \Delta_{\mathrm{T}}^{\star}\left(z_{0}\right)=\Delta_{\mathrm{T}}\left(z_{0}\right)-\mathbf{B}_{\mathrm{T} 1}^{\star}\left(z_{0}\right)-\mathbf{B}_{\mathrm{T} 2}^{\star}\left(z_{0}\right),
$$

where $\Lambda_{\mathrm{T}}\left(z_{0}\right)$ and $\Delta_{\mathrm{T}}\left(z_{0}\right)$ are defined in (2.1). Since the quantities $\mathrm{d}_{0}, \mathrm{H}, \boldsymbol{\Omega}_{02}, \Omega_{20}, \Omega_{22}, \Gamma_{20}$ and $\Gamma_{22}$ are generally unknown, the estimator (4.3) is infeasible in practice. But estimation of these unknown elements has been extensively studied in the literature and similar methods may be utilized in the present context, as we now overview, to produce consistent estimators $\widehat{d}, \widehat{\mathbf{H}}, \widehat{\mathbf{\Omega}}_{02}, \widehat{\Omega}_{20}, \widehat{\mathbf{\Omega}}_{22}, \widehat{\Gamma}_{20}$ and $\widehat{\Gamma}_{22}$, that may be used to construct a feasible version of the FM-kernel estimator.

To simplify, it is convenient to consider the case where the integrated regressors are generated from the cointegrated VAR process (2.3) discussed in Example 1. Then, as in Cheng and Phillips (2009), we may use the Bayesian information criterion to consistently estimate the cointegrating rank $d_{0}$ and use reduced rank regression to consistently estimate (under normalizing restrictions) the matrices $\alpha$ and $\gamma$ in (2.3), and thus obtain a consistent estimator of $\mathbf{H}$ that applies in a general semiparametric setting. Denote the corresponding estimates $\widehat{d}$ and $\widehat{\mathbf{H}}=\left(\widehat{\mathbf{H}}_{1}, \widehat{\mathbf{H}}_{2}\right)$. Feasible FM-kernel estimation further requires estimation of the various long run covariance matrices that appear in (4.1) and (4.2). We illustrate by estimating $\Omega_{20}$. The remaining long run covariance matrix estimates may be constructed in a similar manner. Let

$$
\widehat{e}_{t 0}=Y_{t}-X_{t}^{\prime} \widehat{\beta}(t / T)=Y_{t}-X_{t}^{\prime} \widehat{\beta}_{t}
$$

be the estimated equation errors from kernel regression of (1.1). Let

$$
\widehat{e}_{\mathrm{t} 2}=\widehat{X}_{\mathrm{t} 2}-\widehat{X}_{\mathrm{t}-1,2}=\Delta\left(\widehat{\mathrm{H}}_{2}^{\prime} \mathrm{X}_{\mathrm{t}}\right)
$$

with $\widehat{X}_{t 2}=\widehat{\mathbf{H}}_{2}^{\prime} X_{t}$, and construct estimates of the component autocovariances $\Omega_{20}(j)=E\left[e_{02} e_{j 0}\right]$ using

$$
\widehat{\mathbf{\Omega}}_{20}(\mathbf{j})=\frac{1}{\lfloor(1-\tau) \mathrm{T}\rfloor-\lfloor\tau \mathrm{T}\rfloor} \sum_{\mathrm{t}=\lfloor\tau \mathrm{T}\rfloor+1}^{\lfloor(1-\tau) \mathrm{T}\rfloor} \widehat{e}_{t-j, 2} \widehat{e}_{t 0}, j=-l_{T}, \cdots, 0, \cdots, l_{T},
$$

in which $0<\tau<1 / 2$ is usually close to zero and $l_{T} \ll T$ is the lag truncation number which tends to infinity as $T \rightarrow \infty$. Unlike the existing literature in parametric linear cointegration models where the cross product $\widehat{e}_{t-j, 2} \widehat{e}_{t 0}$ is summed over the full domain of $t$ (i.e., $1 \leqslant t, t-j \leqslant T$ ) to estimate the covariance, our method uses only information over the subinterval from $\lfloor\tau T\rfloor+1$ to $\lfloor(1-\tau) T\rfloor$ to avoid possible boundary effects when applying kernel estimation. Although such construction of covariance estimates may lose 
some useful information by taking $\tau$ close to zero, consistency of the covariance estimate is unaffected. The corresponding long run covariance estimate based on the components (4.4) is

$$
\widehat{\mathbf{\Omega}}_{20}=\sum_{j=-l_{T}}^{l_{T}} k\left(j / l_{T}\right) \widehat{\Omega}_{20}(j),
$$

where $\mathrm{k}(\cdot)$ is a lag kernel function. Using the known uniform consistency of the kernel estimates such as Theorem 4.1 in Li, Phillips and Gao (2016) and following similar arguments to those in the proof of Theorem 4.2 in Phillips, Li and Gao (2017), consistency of $\widehat{\Omega}_{20}$ can be established under mild conditions on the lag kernel function $\mathrm{k}(\cdot)$ and the truncation number $l_{\mathrm{T}}$ (c.f., Phillips, 1995). The proof is standard and details are omitted to save the space.

With consistent estimates of these parameters in hand, we can construct a feasible version of the FMkernel estimator of the coefficient functions. Define a feasible version of the endogeneity correction as

$$
\mathbf{B}_{\mathrm{T} 1}^{\#}\left(z_{0}\right)=\sum_{\mathrm{t}=1}^{\mathrm{T}} \mathrm{K}\left(\frac{\mathrm{t}-\mathrm{T} z_{0}}{\mathrm{Th}}\right) \mathrm{X}_{\mathrm{t}} \odot\left[\mathbf{0}_{\widehat{\mathrm{d}}^{\prime}}^{\prime}\left(\widehat{\boldsymbol{\Omega}}_{02} \widehat{\boldsymbol{\Omega}}_{22}^{+} \Delta \widehat{\mathrm{X}}_{\mathrm{t} 2} \mathbf{1}_{\mathrm{d}-\widehat{\mathrm{d}}}\right)^{\prime}\right]^{\prime},
$$

and a feasible version of the serial correlation correction as

$$
\mathbf{B}_{\mathrm{T} 2}^{\#}\left(z_{0}\right)=\widehat{\mathbf{H}} \overline{\mathbf{Q}}_{\mathrm{T}}^{\#}\left(z_{0}\right) \overline{\mathbf{D}}_{\mathrm{T}}\left\{\mathbf{0}_{\widehat{\mathrm{d}}+1^{\prime}}^{\prime}\left[\mathrm{q}_{\mathrm{T} 2}^{\perp \#}\left(z_{0}\right)^{\prime}\left(\widehat{\boldsymbol{\Gamma}}_{20}-\widehat{\boldsymbol{\Gamma}}_{22} \widehat{\boldsymbol{\Omega}}_{22}^{+} \widehat{\boldsymbol{\Omega}}_{20}\right)\right]^{\prime}\right\}^{\prime},
$$

where $\mathrm{q}_{\mathrm{T} 2}^{\perp \#}\left(z_{0}\right)$ and $\overline{\mathbf{Q}}_{\mathrm{T}}^{\#}\left(z_{0}\right)$ are defined similarly to $\mathrm{q}_{\mathrm{T} 2}^{\perp}\left(z_{0}\right)$ and $\overline{\mathbf{Q}}_{\mathrm{T}}\left(z_{0}\right)$ but with $X_{\mathrm{t} 2}$ replaced by $\widehat{X}_{\mathrm{t} 2 \text {. Using }}$ the corrections (4.6) and (4.7), we propose the feasible FM-kernel estimator

$$
\widehat{\boldsymbol{\beta}}_{\#}\left(z_{0}\right)=\Lambda_{\mathrm{T}}^{+}\left(z_{0}\right) \Delta_{\mathrm{T}}^{\#}\left(z_{0}\right), \quad \Delta_{\mathrm{T}}^{\#}\left(z_{0}\right)=\Delta_{\mathrm{T}}\left(z_{0}\right)-\mathbf{B}_{\mathrm{T} 1}^{\#}\left(z_{0}\right)-\mathbf{B}_{\mathrm{T} 2}^{\#}\left(z_{0}\right),
$$

and proceed to analyze its asymptotic behavior.

Because of the removal of the endogeneity bias via the correction in $\Delta_{\mathrm{T}}^{\#}\left(z_{0}\right)$, the stochastic integral in the limit distribution $\Delta_{2}\left(z_{0}\right)$ is modified accordingly. We define

$$
\Delta_{2}^{\#}\left(z_{0}\right)=\left[\delta^{\#}\left(z_{0}\right), \Delta_{\perp}^{\#}\left(z_{0}\right)^{\prime}\right]^{\prime}
$$

with

$$
\begin{aligned}
& \delta^{\#}\left(z_{0}\right)=\left[2 \mathrm{~B}_{2}\left(z_{0}\right)^{\prime} \mathrm{B}_{2}\left(z_{0}\right)\right]^{1 / 2} \int_{-1}^{1} \mathrm{~K}(z) \mathrm{dB}_{0}^{\#}\left(\frac{z+1}{2}\right), \\
& \Delta_{\perp}^{\#}\left(z_{0}\right)=2 \mathrm{q}_{2}^{\perp}\left(z_{0}\right)^{\prime} \int_{-1}^{1} \mathrm{~K}(z) \mathrm{B}_{2}^{*}\left(\frac{z+1}{2}\right) d \mathrm{~B}_{0}^{\#}\left(\frac{z+1}{2}\right),
\end{aligned}
$$

where the univariate Brownian motion $B_{0}^{\#}(\cdot)$ has (conditional) variance $\omega_{0 \mid 2}=\omega-\Omega_{02} \Omega_{22}^{+} \Omega_{20}$ following the endogeneity correction and is independent of the $\mathrm{d}_{1}$-dimensional Brownian motions $\mathrm{B}_{2}(\cdot)$ and $\mathrm{B}_{2}^{*}(\cdot)$. Hence, the component $\Delta_{2}^{\#}\left(z_{0}\right)$ has a mixed normal distribution which facilitates inference on the time-varying coefficient functions in the same way as the usual FM corrections do in linear cointegrating regression. Noting that the bias correction occurs in the direction $\mathbf{H}_{2}$, the component transform $\mathbf{H}_{1}^{\prime} \widehat{\boldsymbol{\beta}}_{\#}\left(z_{0}\right)$ has the same asymptotic distribution as $\mathbf{H}_{1}^{\prime} \widehat{\boldsymbol{\beta}}\left(z_{0}\right)$. Hence, we only examine the asymptotic distribution of $\widehat{\boldsymbol{\beta}}_{\#}\left(z_{0}\right)$ in the 
direction $\mathbf{H}_{2}$. The following result gives the limit theory in this direction. The asymptotic distribution is mixed normal, giving a nonparametric generalization to the kernel regression case of the original finding in Phillips and Hansen (1990). The asymptotic mixed normality in this direction, coupled with the asymptotic normality in the stationary direction open the way to inference using the FM-kernel estimator.

THEOREM 4. Suppose the conditions of Theorem 1 hold, $\mathrm{T}^{2} \mathrm{~h}^{1+2 \delta_{1}}=\mathrm{o}(1)$, and

$$
\left(\widehat{\mathrm{d}}_{0}, \widehat{\mathbf{H}}, \widehat{\mathbf{\Omega}}_{02}, \widehat{\mathbf{\Omega}}_{20}, \widehat{\mathbf{\Omega}}_{22}, \widehat{\Gamma}_{20}, \widehat{\Gamma}_{22}\right) \rightarrow_{\mathrm{p}}\left(\mathrm{d}_{0}, \mathbf{H}, \mathbf{\Omega}_{02}, \mathbf{\Omega}_{20}, \mathbf{\Omega}_{22}, \Gamma_{20}, \Gamma_{22}\right)
$$

Then, as $T \rightarrow \infty$

$$
\overline{\mathbf{D}}_{\mathrm{T} 2} \overline{\mathbf{Q}}_{\mathrm{T} 2}\left(z_{0}\right)^{\prime} \mathbf{H}_{2}^{\prime}\left[\widehat{\boldsymbol{\beta}}_{\#}\left(z_{0}\right)-\boldsymbol{\beta}\left(z_{0}\right)\right] \Rightarrow \boldsymbol{\Lambda}_{2}^{-1}\left(z_{0}\right) \Delta_{2}^{\#}\left(z_{0}\right)
$$

for fixed $z_{0} \in(0,1)$.

\section{Nonparametric statistical inference}

In practical work interest often focuses on whether time-varying coefficients are well approximated by constant coefficients. To provide an apparatus for formal consideration of such hypotheses this section develops an inferential framework of tests for the coefficient functions in model (1.1) and derives asymptotics that enable formal testing.

\subsection{Testing the global null hypothesis}

As in Section 4, we concentrate on the case of cointegrated regressors. The methodology and theory are similar for the other cases studied in Section 3, and so the details are omitted here. Specifically, we consider testing the following null hypothesis

$$
\mathcal{H}_{0}: \mathbf{R}\left[\boldsymbol{\beta}(z)-\boldsymbol{\beta}_{0}\right]=\mathbf{0}_{\mathrm{r}}
$$

holds for any $0<z<1$, where $\mathbf{R}$ is an $\boldsymbol{r} \times \mathrm{d}$ restriction matrix of rank $\boldsymbol{r}<\mathrm{d}$ and $\boldsymbol{\beta}_{0}$ is a d-dimensional vector of unknown parameters. As $\mathbf{R}$ does not rely on $z$, we refer to $\mathcal{H}_{0}$ as a global null hypothesis.

Before developing a statistic for testing the hypothesis $\mathcal{H}_{0}$, we derive a useful result from the limit distributions given in Theorem 1. From (2.12) and (2.13) and as in Remark 2(e), we note that

$$
\sqrt{\mathrm{Th}}\left[\widehat{\boldsymbol{\beta}}\left(z_{0}\right)-\boldsymbol{\beta}\left(z_{0}\right)\right] \Rightarrow \mathbf{H}_{1} \xi,
$$

under the assumptions of Theorem 1, where $\xi$ is the Gaussian vector defined in Theorem 1(i). Further, under Assumption $1^{*}$, the vector $\xi$ is a $d_{0}$-dimensional centred normal vector with covariance matrix $\nu_{0} \sigma_{e}^{2} \Lambda_{11}^{+}$, where $\sigma_{e}^{2}$ is defined in Assumption $1^{*}$. The covariance matrix of $\mathbf{H}_{1} \xi$ is therefore $v_{0} \sigma_{e}^{2} \mathbf{H}_{1} \boldsymbol{\Lambda}_{11}^{+} \mathbf{H}_{1}^{\prime}$, which has degenerate rank.

Construction of a test statistic based on (5.1) requires consistent estimation of the unknown elements $\sigma_{e}^{2}$ and $\mathbf{H}_{1} \boldsymbol{\Lambda}_{11}^{+} \mathbf{H}_{1}^{\prime}$ in the covariance structure. From Phillips (1988) and using Proposition 1 in Section 2, we may 
use the matrix $\left[\frac{1}{T h} \sum_{t=1}^{T} X_{t} X_{t}^{\prime} K\left(\frac{t-T z}{T h}\right)\right]^{+}$as an estimate of $\mathbf{H}_{1} \Lambda_{11}^{+} \mathbf{H}_{1}^{\prime}$ in view of the fact that

$$
\left[\frac{1}{\mathrm{Th}} \sum_{\mathrm{t}=1}^{\mathrm{T}} \mathrm{X}_{\mathrm{t}} \mathrm{X}_{\mathrm{t}}^{\prime} \mathrm{K}\left(\frac{\mathrm{t}-\mathrm{Tz}}{\mathrm{Th}}\right)\right]^{+}=\mathrm{H}_{1} \boldsymbol{\Lambda}_{11}^{+} \mathbf{H}_{1}^{\prime}+\mathrm{op}_{\mathrm{P}}(1), \mathrm{h} \leqslant z \leqslant 1-\mathrm{h} .
$$

The above convergence holds uniformly over $h \leqslant z \leqslant 1-h$. Let the residual $\widehat{e}_{t 0}$ be defined as in Section 4 , and construct the equation error variance estimate

$$
\widehat{\sigma}_{e}^{2}=\frac{1}{\lfloor(1-\tau) T\rfloor-\lfloor\tau T\rfloor} \sum_{t=\lfloor\tau T\rfloor+1}^{\lfloor(1-\tau) T\rfloor} \widehat{e}_{t 0}^{2}
$$

where $\tau$ is defined as in Section 4, which gives a consistent estimate of $\sigma_{e}^{2}$ in view of Theorem 4.1 in Li, Phillips and Gao (2016), so that

$$
\widehat{\sigma}_{e}^{2}=\sigma_{e}^{2}+o_{P}(1)
$$

Next let $\left\{z_{\mathrm{k}}\right\}_{\mathrm{k}=1}^{\mathrm{m}}$ be an equidistant grid of points that satisfy $0<\mathrm{h}=z_{1}<z_{2}<\cdots<z_{\mathrm{m}-1}<z_{\mathrm{m}}=1-\mathrm{h}$ and are chosen from the interval $(0,1)$, where the number $m$ is a fixed positive integer. The extension to divergent $m$ will be discussed later in Remark 7. Using (5.1)-(5.3), we construct point-wise Wald test statistics of $\mathcal{H}_{0}$ as

$$
W_{\mathrm{T}}\left(z_{\mathrm{k}}\right)=(\mathrm{Th})\left\{\mathbf{R}\left[\widehat{\boldsymbol{\beta}}\left(z_{\mathrm{k}}\right)-\widehat{\boldsymbol{\beta}}\right]\right\}^{\prime}\left\{\widehat{\boldsymbol{\sigma}}_{e}^{2} v_{0} \mathbf{R}\left[\frac{1}{\mathrm{Th}} \sum_{\mathrm{t}=1}^{\mathrm{T}} \mathrm{X}_{\mathrm{t}} X_{\mathrm{t}}^{\prime} \mathrm{K}\left(\frac{\mathrm{t}-\mathrm{T} z_{\mathrm{k}}}{\mathrm{Th}}\right)\right]^{+} \mathbf{R}^{\prime}\right\}^{+}\left\{\mathbf{R}\left[\widehat{\boldsymbol{\beta}}\left(z_{\mathrm{k}}\right)-\widehat{\boldsymbol{\beta}}\right]\right\},
$$

where $\widehat{\beta}$ is a conventional parametric estimate of $\beta_{0}$ under the global null hypothesis $\mathcal{H}_{0}$. Assume that

$$
\operatorname{rank}\left(\mathbf{R H}_{1} \boldsymbol{\Lambda}_{11}^{+} \mathbf{H}_{1}^{\prime} \mathbf{R}^{\prime}\right)=\mathrm{r}
$$

and that under the null

$$
\widehat{\boldsymbol{\beta}}-\boldsymbol{\beta}_{0}=\mathrm{o}_{\mathrm{P}}(1 / \sqrt{\mathrm{Th}}) .
$$

It is natural to propose a generalized Wald test statistic by summing the component statistics $W_{\mathrm{T}}\left(z_{\mathrm{k}}\right)$ over $k=1, \cdots$, m giving

$$
\mathrm{W}_{\mathrm{T}}=\sum_{\mathrm{k}=1}^{\mathrm{m}} \mathrm{W}_{\mathrm{T}}\left(z_{\mathrm{k}}\right)
$$

The following theorem gives the limit distribution of the generalized Wald test statistic.

THEOREM 5. Suppose the conditions of Theorem 1, Assumption $1^{*},(5.5),(5.6)$, and $\mathrm{Th}^{1+2 \delta_{1}}=\mathrm{o}(1)$ hold. Letting the positive integer $m$ be fixed, we have $W_{\mathrm{T}} \Rightarrow \chi_{\mathrm{m} r}^{2}$ under the null hypothesis $\mathcal{H}_{0}$, where $\chi_{\mathrm{mr}}^{2}$ is a central chi-square distribution with ( $\mathrm{mr}$ ) degrees of freedom. In addition, if (4.9) holds, $\mathrm{W}_{\mathrm{T}}^{\#} \Rightarrow \chi_{\mathrm{mr}}^{2}$ under the null hypothesis $\mathcal{H}_{0}$, where $\mathrm{W}_{\mathrm{T}}^{\#}$ is constructed in the same manner as $\mathrm{W}_{\mathrm{T}}$ but using FM-kernel estimates of the time-varying coefficients.

REMARK 7. (a) The methodology and theory developed above are applicable if we generalize the global null hypothesis $\mathcal{H}_{0}$ to

$$
\mathcal{H}_{0}^{\star}: \mathbf{R}[\boldsymbol{\beta}(z)-\boldsymbol{\beta}(z, \zeta)]=\mathbf{0}_{\mathrm{r}}
$$


holds for any $0<z<1$, where $\beta(\cdot, \zeta)=\left[\beta_{1}\left(\cdot, \zeta_{1}\right), \cdots, \beta_{d}\left(\cdot, \zeta_{d}\right)\right]^{\prime}, \beta_{\mathfrak{i}}\left(\cdot, \zeta_{\mathfrak{i}}\right), \mathfrak{i}=1, \cdots, d$, are pre-specified nonlinear functional coefficients with $\zeta_{i}$ being an unknown parameter vector, $\zeta=\left(\zeta_{1}^{\prime}, \ldots, \zeta_{d}^{\prime}\right)^{\prime}$. We let $\widehat{\zeta}$ be the conventional nonlinear least squares estimator of the parameter vector $\zeta$ and correspondingly construct the generalized Wald test statistic as $\widehat{W}_{\mathrm{T}}=\sum_{\mathrm{k}=1}^{\mathrm{m}} \widehat{W}_{\mathrm{T}}\left(z_{\mathrm{k}}\right)$, where

$$
\widehat{W}_{\mathrm{T}}\left(z_{\mathrm{k}}\right)=\widehat{\mathbf{R}}_{\boldsymbol{\beta}}\left(z_{\mathrm{k}}, \widehat{\zeta}\right)^{\prime}\left\{\widehat{\sigma}_{\mathrm{e}}^{2} v_{0} \mathbf{R}\left[\frac{1}{\mathrm{Th}} \sum_{\mathrm{t}=1}^{\mathrm{T}} \mathrm{X}_{\mathrm{t}} \mathrm{X}_{\mathrm{t}}^{\prime} \mathrm{K}\left(\frac{\mathrm{t}-\mathrm{T} z_{\mathrm{k}}}{\mathrm{Th}}\right)\right]^{+} \mathbf{R}^{\prime}\right\}^{+} \widehat{\mathbf{R}}_{\boldsymbol{\beta}}\left(z_{\mathrm{k}}, \widehat{\zeta}\right)
$$

with

$$
\widehat{\mathbf{R}}_{\boldsymbol{\beta}}\left(z_{k}, \widehat{\zeta}\right)=(T h)^{1 / 2} \mathbf{R}\left[\widehat{\boldsymbol{\beta}}\left(z_{k}\right)-\boldsymbol{\beta}\left(z_{k}, \widehat{\zeta}\right)\right]
$$

By replacing (5.6) by $\widehat{\zeta}-\zeta=\mathrm{o}_{\mathrm{P}}(1 / \sqrt{\mathrm{Th}})$ and imposing appropriate smoothness condition on the prespecified functional coefficients $\beta(\cdot, \cdot)$, we may show the limit distribution of $\widehat{W}_{\mathrm{T}}$ is similar to those given in Theorem 5.

(b) We next briefly discuss the case that $m$ diverges to infinity as $T \rightarrow \infty$. Using (5.2) and (5.3), we may prove that

$$
W_{\mathrm{T}}\left(z_{\mathrm{k}}\right)=(\mathrm{Th})\left[\widehat{\boldsymbol{\beta}}\left(z_{\mathrm{k}}\right)-\boldsymbol{\beta}_{0}\right]^{\prime} \mathbf{R}^{\prime}\left[\sigma_{\mathrm{e}}^{2} v_{0} \mathbf{R} \mathbf{H}_{1} \boldsymbol{\Lambda}_{11}^{+} \mathbf{H}_{1}^{\prime} \mathbf{R}^{\prime}\right]^{+} \mathbf{R}\left[\widehat{\boldsymbol{\beta}}\left(z_{\mathrm{k}}\right)-\boldsymbol{\beta}_{0}\right]\left(1+\mathrm{o}_{\mathrm{P}}(1)\right)
$$

Following the proof of Theorem 1 in Appendix A and noting that the kernel function $K(\cdot)$ has the compact support $[0,1], \widehat{\boldsymbol{\beta}}\left(z_{\mathrm{k}}\right)-\boldsymbol{\beta}_{0}$ is asymptotically determined by $\left(e_{\mathrm{t} 0}, e_{\mathrm{t} 1}\right), \mathrm{T}\left(z_{\mathrm{k}}-\mathrm{h}\right) \leqslant \mathrm{t} \leqslant \mathrm{T}\left(z_{\mathrm{k}}+\mathrm{h}\right)$. By (5.9) and Assumption 1 in Section 2.1, we may show that $\left\{W_{\mathrm{T}}\left(z_{\mathrm{k}}\right)\right\}_{\mathrm{k}=1}^{\mathrm{m}}$ is a sequence of asymptotically independent random elements when $z_{k+1}-z_{k} \geqslant 2 h$. So the generalized Wald test statistic $W_{\mathrm{T}}$ can be viewed as a sum of asymptotically independent random variables. By appropriately centralizing $W_{\mathrm{T}}$ and using standard central limit arguments, it is clear that the generalized Wald statistic is asymptotically normal when $\mathrm{m} \rightarrow \infty$.

\subsection{Testing the local null hypothesis}

We next turn to the more challenging case where the rank condition (5.5) fails. Our approach follows Phillips (1995) in the application of FM regression to models with cointegrated variates where Wald test statistics suffer from rank condition failure asymptotically. As will become apparent, kernel FM regression tests involve further complications under rank condition failure in (5.5).

To proceed, we consider the localized version of the null hypothesis:

$$
\mathcal{H}_{0}^{\diamond}: \mathbf{R}(z)\left[\beta(z)-\beta_{0}\right]=\mathbf{0}_{\mathrm{r}}
$$

holds for any $0<z<1$, where $\mathbf{R}(z)$ is an $r \times d$ time-varying restriction matrix. As discussed in Phillips (1995) rank condition failure occurs when the restriction matrix isolates some of the nonstationary variable coefficients, thereby necessarily involving estimates of these coefficients in the limit theory of Wald-type test statistics. Motivated by Phillips (1995), under such rank condition failure, the restriction matrix can be 
written in the following form

$$
\begin{aligned}
\mathbf{R}(z)^{\prime} & =\left[\mathbf{R}_{1}(z) \vdots \mathbf{R}_{2}(z)\right]=\left(\mathbf{H}_{1}, \mathbf{H}_{2}\right)\left[\begin{array}{cccc}
\mathbf{S}_{1} & \mathbf{S}_{\mathrm{h} 1} & \vdots & \mathbf{O}_{\mathrm{d}_{0} \times \mathrm{r}_{2}} \\
\mathbf{O}_{\mathrm{d}_{1} \times \mathrm{r}_{0}} & \overline{\mathbf{Q}}_{\mathrm{T} 2}(z) \mathbf{S}_{\mathrm{h} 2} & \vdots & \overline{\mathbf{Q}}_{\mathrm{T} 2}(z) \boldsymbol{S}_{2}
\end{array}\right] \\
& =\left[\mathbf{H}_{1} \mathbf{S}_{1}, \mathbf{H}_{1} \boldsymbol{S}_{\mathrm{h} 1}+\mathbf{H}_{2} \overline{\mathbf{Q}}_{\mathrm{T} 2}(z) \mathbf{S}_{\mathrm{h} 2} \vdots \mathbf{H}_{2} \overline{\mathbf{Q}}_{\mathrm{T} 2}(z) \boldsymbol{S}_{2}\right],
\end{aligned}
$$

which in the present case involves the localized structure where $R_{1}(z)$ and $R_{2}(z)$ are $d \times r_{1}$ and $d \times r_{2}$ matrices with $r_{1}+r_{2}=r, S_{1}, S_{h 1}, S_{h 2}$ and $S_{2}$ are the matrices with sizes $d_{0} \times r_{0}, d_{0} \times\left(r_{1}-r_{0}\right), d_{1} \times\left(r_{1}-r_{0}\right)$ and $d_{1} \times r_{2}$, respectively, $S_{h 1}$ has full column rank. From (5.11), we have that (5.5) reduces as follows

$$
\mathbf{R}(z) \mathbf{H}_{1} \boldsymbol{\Lambda}_{11}^{+} \mathbf{H}_{1}^{\prime} \mathbf{R}(z)^{\prime}=\left[\begin{array}{cc}
\mathbf{R}_{1}(z) \mathbf{H}_{1} \boldsymbol{\Lambda}_{11}^{+} \mathbf{H}_{1}^{\prime} \mathbf{R}_{1}(z)^{\prime} & \mathbf{O}_{\mathrm{r}_{1} \times \mathrm{r}_{2}} \\
\mathbf{O}_{\mathrm{r}_{2} \times \mathrm{r}_{1}} & \mathbf{O}_{\mathrm{r}_{2} \times \mathrm{r}_{2}}
\end{array}\right]
$$

whose rank is smaller than $r$.

The rank deficiency in (5.12) implies that the arguments used above to prove Theorem 5 no longer apply to the generalized Wald statistic for testing the $\mathcal{H}_{0}^{\diamond}$ and different methods are required. Instead of using Theorem 1 in Section 2, we make use of Theorem 4 in Section 4. In the remainder of this section, we apply the test statistic constructed from the FM-kernel estimates for which the mixed normal distribution derived in Theorem 4 plays an important role in achieving the limit theory. Further, to simplify derivations, we use the uniform kernel $\mathrm{K}(\mathrm{u})=\mathrm{I}(-1 \leqslant u \leqslant 1)$ where $\mathrm{I}(\cdot)$ denotes the indicator function. We remark that $\mu_{0}=2$ in this case, which differs from the unit normalization condition used in Assumption 3(ii).

Again, we start by defining the following point-wise Wald statistics based on FM-kernel estimation

$$
W_{\mathrm{T}}^{\diamond}\left(z_{\mathrm{k}}\right)=\widehat{\mathbf{R}}_{\#}\left(z_{\mathrm{k}}\right)^{\prime}\left\{\widehat{\sigma}_{\mathrm{e}}^{2} v_{0} \mathbf{R}\left(z_{\mathrm{k}}\right)\left[\frac{1}{\mathrm{Th}} \sum_{\mathrm{t}=1}^{\mathrm{T}} X_{\mathrm{t}} X_{\mathrm{t}}^{\prime} \mathrm{K}\left(\frac{\mathrm{t}-\mathrm{T} z_{\mathrm{k}}}{\mathrm{Th}}\right)\right]^{+} \mathbf{R}\left(z_{\mathrm{k}}\right)^{\prime}\right\}^{+} \widehat{\mathbf{R}}_{\#}\left(z_{\mathrm{k}}\right)
$$

where

$$
\widehat{\mathbf{R}}_{\#}\left(z_{\mathrm{k}}\right)=(\mathrm{Th})^{1 / 2} \mathbf{R}\left(z_{\mathrm{k}}\right)\left[\widehat{\boldsymbol{\beta}}_{\#}\left(z_{\mathrm{k}}\right)-\widehat{\boldsymbol{\beta}}\right],
$$

and $v_{0}=2$ since $K(u)=I(-1 \leqslant u \leqslant 1)$. As in (5.7), we construct the generalized Wald test statistic by summing $W_{\mathrm{T}}^{\diamond}\left(z_{k}\right)$ over $k=1, \cdots, m$, i.e.,

$$
\mathrm{W}_{\mathrm{T}}^{\diamond}=\sum_{\mathrm{k}=1}^{\mathrm{m}} \mathrm{W}_{\mathrm{T}}^{\diamond}\left(z_{\mathrm{k}}\right)
$$

Define

$$
\overline{\mathbf{D}}_{\mathrm{T}}=\operatorname{diag}\left\{\mathbf{I}_{\mathrm{d}_{0},} \sqrt{\mathrm{T}},(\sqrt{\mathrm{Th}}) \mathbf{I}_{\mathrm{d}_{1}-1}\right\}
$$

and assume

$$
\sqrt{\operatorname{Th}} \overline{\mathbf{D}}_{\mathrm{T}}^{\diamond} \mathbf{H}^{\prime}\left(\widehat{\boldsymbol{\beta}}-\boldsymbol{\beta}_{0}\right)=\mathrm{oP}_{\mathrm{P}}(1) .
$$

The following limit theorem provides the asymptotic distribution of $W_{\mathrm{T}}^{\diamond}$ defined in (5.14) under the local null hypothesis, which differs from the earlier limit distribution given in (5.10) and can be viewed as a nonparametric kernel-FM test generalization of Theorem 4.5 in Phillips (1995). 
THEOREM 6. Suppose that the conditions of Theorem 1, Assumption $1^{*},(5.15)$ and $\mathrm{T}^{2} \mathrm{~h}^{1+2 \delta_{1}}=\mathrm{o}(1)$ are all satisfied. Letting the positive integer $m$ be fixed, we have

$$
W_{\mathrm{T}}^{\diamond} \Rightarrow \chi_{m r_{1}}^{2}+\frac{\omega_{0 \mid 2}}{\sigma_{e}^{2}} \chi_{m r_{2, * \prime}}^{2}
$$

under the null hypothesis $\mathcal{H}_{0}^{\diamond}$ with (5.11), where $\chi_{m r_{1}}^{2}$ and $\chi_{m r_{2}, *}^{2}$ are two independent chi-square distributions with degrees of freedom $\mathrm{mr}_{1}$ and $\mathrm{mr}_{2}$, respectively, and $\omega_{0 \mid 2}=\omega-\Omega_{02} \Omega_{22}^{+} \Omega_{20}$.

REMARK 8. Note that $\omega_{0 \mid 2}=\omega-\Omega_{02} \Omega_{22}^{+} \Omega_{20} \leqslant \omega$ so that the ratio in (5.16) can be written in the form

$$
\frac{\omega_{0 \mid 2}}{\sigma_{e}^{2}}=\frac{\omega-\Omega_{02} \Omega_{22}^{+} \Omega_{20}}{\omega} \cdot \frac{\omega}{\sigma_{e}^{2}} \leqslant \frac{\omega}{\sigma_{e}^{2}} .
$$

It follows that

$$
\mathrm{P}\left(\mathrm{W}_{\mathrm{T}}^{\diamond}>w\right) \rightarrow \mathrm{P}\left(\chi_{\mathrm{m} r_{1}}^{2}+\frac{\omega_{0 \mid 2}}{\sigma_{e}^{2}} \chi_{m r_{2, *}}^{2}>w\right) \leqslant \mathrm{P}\left(\chi_{m r_{1}}^{2}+\frac{\omega}{\sigma_{e}^{2}} \chi_{m r_{2, *}}^{2}>w\right),
$$

so that a test of $\mathcal{H}_{0}^{\diamond}$ based on critical values of the distribution of $\chi_{m r_{1}}^{2}+\frac{\omega}{\sigma_{e}^{2}} \chi_{m r_{2, *}}^{2}$ would be an asymptotically conservative test if $r_{1}$ and $r_{2}$ were known and consistent estimates of $\omega=\operatorname{lrvar}\left(e_{t 0}\right)$ and $\sigma_{e}^{2}$ were employed in calculating critical values. Further, under Assumption $1^{*}$, we have $\omega_{0 \mid 2}=\sigma_{e}^{2}-\Omega_{02} \Omega_{22}^{+} \Omega_{20} \leqslant \sigma_{e}^{2}$ and then

$$
\chi_{m r_{1}}^{2}+\frac{\omega}{\sigma_{e}^{2}} \chi_{m r_{2, *}}^{2} \leqslant \chi_{m r_{1}}^{2}+\chi_{m r_{2, *}}^{2}={ }_{d} \chi_{m r}^{2}
$$

so that a conservative test can be computed directly by using critical values from a $\chi_{m r}^{2}$ distribution.

\section{Monte-Carlo simulations}

This section reports Monte-Carlo simulations designed to examine the finite sample performance of the proposed methods and illustrate the kernel estimation and inferential limit theory given in Sections $2-5$. It is well known that the numerical performance of kernel-based local regression estimation relies on the choice of bandwidth. In the classic nonparametric regression setting with independent or stationary regressors, crossvalidation $(\mathrm{CV})$ is probably the most commonly-used method to select the bandwidth and its asymptotic optimality property has been extensively studied in the literature. However, bandwidth selection is a challenging issue in the time-varying coefficient cointegrating regression framework of the present paper. From the asymptotic theory developed in Sections 2 and 3 the kernel estimators of the coefficient functions have different convergence rates in different directions, indicating that optimal bandwidth orders vary in these different directions. In consequence use of a universal optimal bandwidth determined by a method such as cross validation may not perform well in finite samples. In the following simulation studies we therefore employ different rules to select bandwidths in different settings, which helps to ensure reliable numerical performance of the kernel estimation procedure in the present setting.

EXAMPLE 6.1. (i) Let $d=3$ and consider the data generating process:

$$
Y_{t}=\beta_{t}^{\prime} X_{t}+e_{t 0}, t=1, \cdots, T,
$$


where $X_{t}=\left(X_{t 1}, X_{t 2}^{\prime}\right)^{\prime}, X_{t 1}=e_{t 1}, \Delta X_{t 2}=\left(e_{t 2}, e_{t 3}\right)^{\prime}$, the process $e_{t}=\left(e_{t 1}, e_{t 2}, e_{t 3}\right)^{\prime}$ is independently generated from a three-dimensional normal distribution with mean zero and variance matrix

$$
\Omega_{1}=\left(\begin{array}{lll}
1 & \rho & \rho \\
\rho & 1 & \rho \\
\rho & \rho & 1
\end{array}\right), \rho=0,0.2 \text { or } 0.4
$$

The error term $e_{\mathrm{t} 0}$ is independently generated from $\mathrm{N}\left(0,0.5^{2}\right)$ and is independent of $e_{\mathrm{t}}$, and the following coefficient functions $\beta_{t}=\beta(t / T)=\left(\beta_{t 1}, \beta_{t 2}, \beta_{t 3}\right)^{\prime}$ are defined as

$$
\beta_{\mathrm{t} 1}=\beta_{1}(\mathrm{t} / \mathrm{T})=1+\mathrm{t} / \mathrm{T}, \quad \beta_{\mathrm{t} 2}=\beta_{2}(\mathrm{t} / \mathrm{T})=\sqrt{1+\mathrm{t} / \mathrm{T}}, \quad \beta_{\mathrm{t} 3}=\beta_{3}(\mathrm{t} / \mathrm{T})=1+(\mathrm{t} / \mathrm{T})^{2} .
$$

are employed giving linear, square root and quadratic specifications. When $\rho=0.2$ or 0.4 , the stationary component $X_{\mathrm{t} 1}$ is correlated with the nonstationary component $X_{\mathrm{t} 2}$. We use the local kernel estimation technique $\widehat{\beta}(z)=\left[\widehat{\beta}_{1}(z), \widehat{\beta}_{2}(z), \widehat{\beta}_{3}(z)\right]^{\prime}$ to estimate the coefficient functions $\boldsymbol{\beta}(z)$, where the Epanechnikov kernel $\mathrm{K}(\mathrm{u})=\frac{3}{4}\left(1-\mathrm{u}^{2}\right) \mathrm{I}(-1 \leqslant u \leqslant 1)$ is used and the bandwidth is $h=0.8 \mathrm{~h}_{\mathrm{cv}}$ with $\mathrm{h}_{\mathrm{c} v}$ determined by the CV selection criterion. The reason for slightly scaling down the CV selected bandwidth $h_{c v}$ in the kernel estimation procedure is partly due to the bandwidth restriction in Theorem 1, which requires undersmoothing to make the kernel estimation bias asymptotically negligible.

This simulation design serves to illustrate the kernel estimation theory in Section 2. From the above data generating scheme, the global rotation matrix is $\mathbf{H}=\mathbf{I}_{3}$ and the local rotation matrix is $\overline{\mathbf{Q}}_{\mathrm{T}}(z)=$ $\operatorname{diag}\left\{1, \mathbf{Q}_{\mathrm{T} 2}(z)\right\}$, where $\mathbf{Q}_{\mathrm{T} 2}(z)=\left[\mathrm{q}_{\mathrm{T} 2}(z), \mathrm{q}_{\mathrm{T} 2}^{\perp}(z)\right]$ with

$$
\begin{aligned}
& \mathrm{q}_{\mathrm{T} 2}(z)=X_{\lfloor\mathrm{T}(z-h)\rfloor, 2} /\left\|X_{\lfloor\mathrm{T}(z-h)\rfloor, 2}\right\|=:\left[\mathrm{p}_{1}(z), \mathrm{p}_{2}(z)\right]^{\prime}, \\
& \mathrm{q}_{\mathrm{T} 2}^{\perp}(z)=\left[\mathrm{p}_{2}(z),-\mathrm{p}_{1}(z)\right]^{\prime} .
\end{aligned}
$$

From the definition of the rotation matrices, the kernel estimates in the three different directions (as discussed in Section 2) can be expressed as

$$
\widehat{\beta}_{1}(z), p_{1}(z) \widehat{\beta}_{2}(z)+p_{2}(z) \widehat{\beta}_{3}(z), p_{2}(z) \widehat{\beta}_{2}(z)-p_{1}(z) \widehat{\beta}_{3}(z),
$$

respectively. Their corresponding rates of convergence are given in (2.15)-(2.17), respectively. In the simulation, we consider three interior points in the kernel estimation $(z=0.25,0.5,0.75)$. For the point-wise kernel estimates at these points, we calculate the respective estimated standard errors over 1000 replications of the following transformed and centered quantities:

$$
\begin{aligned}
& g_{\mathrm{T} 1}^{1}(z)=\widehat{\beta}_{1}(z)-\beta_{1}(z), \\
& g_{\mathrm{T} 2}^{1}(z)=p_{1}(z)\left[\widehat{\beta}_{2}(z)-\beta_{2}(z)\right]+p_{2}(z)\left[\widehat{\beta}_{3}(z)-\beta_{3}(z)\right], \\
& g_{\mathrm{T} 3}^{1}(z)=p_{2}(z)\left[\widehat{\beta}_{2}(z)-\beta_{2}(z)\right]-p_{1}(z)\left[\widehat{\beta}_{3}(z)-\beta_{3}(z)\right] .
\end{aligned}
$$

In what follows, the affix $j$ in the notation $g_{T 1}^{j}(z)$ indicates the relevant experiment; and in the present case $j=1$ with corresponding results given in Table 1 .

We observe from Table 1 that all estimated standard errors decrease as the sample size $\mathrm{T}$ increases. 
Table 1: Standard errors of kernel estimates in three different directions for setting (i)

\begin{tabular}{|c|c|c|c|c|c|c|c|c|c|c|}
\hline \multirow[b]{2}{*}{$\mathrm{T}$} & \multirow[t]{2}{*}{$z$} & \multicolumn{3}{|c|}{$\rho=0$} & \multicolumn{3}{|c|}{$\rho=0.2$} & \multicolumn{3}{|c|}{$\rho=0.4$} \\
\hline & & 200 & 600 & 1000 & 200 & 600 & 1000 & 200 & 600 & 1000 \\
\hline \multirow{3}{*}{$g_{\mathrm{T} 1}^{1}(z)$} & 0.25 & 0.1554 & 0.1248 & 0.1134 & 0.1592 & 0.1234 & 0.1083 & 0.1675 & 0.1246 & 0.1147 \\
\hline & 0.50 & 0.1571 & 0.1322 & 0.1183 & 0.1681 & 0.1331 & 0.1201 & 0.1626 & 0.1290 & 0.1187 \\
\hline & 0.75 & 0.1822 & 0.1423 & 0.1189 & 0.1792 & 0.1443 & 0.1247 & 0.1839 & 0.1390 & 0.1228 \\
\hline \multirow{3}{*}{$g_{\mathrm{T} 2}^{1}(z)$} & 0.25 & 0.0376 & 0.0189 & 0.0132 & 0.0430 & 0.0179 & 0.0147 & 0.0402 & 0.0191 & 0.0133 \\
\hline & 0.50 & 0.0245 & 0.0120 & 0.0095 & 0.0284 & 0.0142 & 0.0102 & 0.0269 & 0.0147 & 0.0102 \\
\hline & 0.75 & 0.0206 & 0.0101 & 0.0074 & 0.0234 & 0.0114 & 0.0079 & 0.0270 & 0.0120 & 0.0081 \\
\hline \multirow{3}{*}{$\mathrm{g}_{\mathrm{T} 3}^{1}(z)$} & 0.25 & 0.1294 & 0.0888 & 0.0763 & 0.1324 & 0.0984 & 0.0767 & 0.1590 & 0.1150 & 0.0947 \\
\hline & 0.50 & 0.1158 & 0.0819 & 0.0689 & 0.1179 & 0.0743 & 0.0727 & 0.1246 & 0.0900 & 0.0706 \\
\hline & 0.75 & 0.1638 & 0.1067 & 0.0865 & 0.1523 & 0.1113 & 0.0837 & 0.1664 & 0.1152 & 0.0864 \\
\hline
\end{tabular}

Broadly speaking, the standard errors of $g_{\mathrm{T} 2}^{1}(\cdot)$ are much smaller than those of $g_{\mathrm{T} 3}^{1}(\cdot)$, and the standard errors of $g_{\mathrm{T} 1}^{1}$ (in the stationary direction) have the largest values. These results generally support the limit theory and relative convergence rates obtained in Section 2 where $g_{\mathrm{T} 1}^{1}(z)=\mathrm{O}_{\mathrm{P}}\left(\frac{1}{\sqrt{\mathrm{Th}}}\right), g_{\mathrm{T} 2}^{1}(z)=\mathrm{O}_{\mathrm{P}}\left(\frac{1}{\mathrm{~T} \sqrt{\mathrm{h}}}\right)$, and $\mathrm{g}_{\mathrm{T} 3}^{1}(z)=\mathrm{O}_{\mathrm{P}}\left(\frac{1}{\mathrm{Th}}\right)$ for fixed $z \in(0,1)$. Also, we observe that correlation between the stationary component $X_{t 1}$ and the nonstationary component $X_{t 2}$ does not noticeably impact the finite sample estimation results.

(ii) We next let $d=2$ and consider mixed stochastic and deterministic trends in generating the nonstationary regressors. Define

$$
Y_{t}=\beta_{t}^{\prime} X_{t}+e_{t 0}, t=1, \cdots, T,
$$

where $X_{t}=X_{t-1}+\boldsymbol{\mu}+u_{t}, \boldsymbol{\mu}=(0.1,0.2)^{\prime}$, the initial value $X_{0}=\mathbf{0}_{2}, \mathfrak{u}_{t}$ is independently generated as bivariate normal with mean zero and

$$
\Omega_{2}=\left(\begin{array}{ll}
1 & \rho \\
\rho & 1
\end{array}\right), \rho=0,0.2 \text { or } 0.5,
$$

the error term $e_{\mathrm{t} 0}$ is defined as in setting (i), and the coefficient functions $\beta_{\mathrm{t}}=\boldsymbol{\beta}(\mathrm{t} / \mathrm{T})=\left(\beta_{\mathrm{t} 1}, \beta_{\mathrm{t} 2}\right)^{\prime}$ are defined by the linear and quadratic functions

$$
\beta_{\mathrm{t} 1}=\beta_{1}(\mathrm{t} / \mathrm{T})=1+\mathrm{t} / \mathrm{T}, \beta_{\mathrm{t} 2}=\beta_{2}(\mathrm{t} / \mathrm{T})=1+(\mathrm{t} / \mathrm{T})^{2} .
$$

The aim of this design is to assess the asymptotic theory of Section 3.1 when the nonstationary regressors have a mixture of stochastic and deterministic trends. From the data generating scheme, we have the local rotation matrix $\widetilde{\mathbf{Q}}_{\mathrm{T}}(z)=\left[\widetilde{\mathrm{q}}_{\mathrm{T}}(z), \widetilde{\mathrm{q}}_{\mathrm{T}}^{\perp}(z)\right]$ with

$$
\begin{aligned}
\widetilde{\mathrm{q}}_{\mathrm{T}}(z) & =\mathrm{X}_{\lfloor\mathrm{T}(z-\mathrm{h})\rfloor} /\left\|\mathrm{X}_{\lfloor\mathrm{T}(z-\mathrm{h})\rfloor}\right\|=:\left[\widetilde{\mathrm{p}}_{1}(z), \widetilde{\mathrm{p}}_{2}(z)\right]^{\prime} \\
\widetilde{\mathrm{q}}_{\mathrm{T}}^{\perp}(z) & =\left[\widetilde{\mathrm{p}}_{2}(z),-\widetilde{\mathrm{p}}_{1}(z)\right]^{\prime} .
\end{aligned}
$$

Let $\widehat{\beta}(z)=\left[\widehat{\beta}_{1}(z), \widehat{\beta}_{2}(z)\right]^{\prime}$ be the kernel regression estimator of $\beta(z)$. The kernel estimates in the two different directions (as discussed in Section 3.1) are

$$
\widetilde{p}_{1}(z) \widehat{\beta}_{1}(z)+\widetilde{p}_{2}(z) \widehat{\beta}_{2}(z) \text { and } \widetilde{p}_{2}(z) \widehat{\beta}_{1}(z)-\widetilde{p}_{1}(z) \widehat{\beta}_{2}(z),
$$

respectively. They have the convergence rates given in (3.11) and (3.12). We use the same interior points 
Table 2: Standard errors of kernel estimates in two different directions for setting (ii)

\begin{tabular}{|c|c|c|c|c|c|c|c|c|c|c|}
\hline \multirow[b]{2}{*}{$\mathrm{n}$} & \multirow[t]{2}{*}{$z$} & \multicolumn{3}{|c|}{$\rho=0$} & \multicolumn{3}{|c|}{$\rho=0.2$} & \multicolumn{3}{|c|}{$\rho=0.5$} \\
\hline & & 200 & 600 & 1000 & 200 & 600 & 1000 & 200 & 600 & 1000 \\
\hline \multirow{3}{*}{$g_{\mathrm{T} 1}^{2}(z)$} & 0.25 & 0.0509 & 0.0091 & 0.0047 & 0.0568 & 0.0110 & 0.0052 & 0.0529 & 0.0125 & 0.0053 \\
\hline & 0.50 & 0.0270 & 0.0045 & 0.0026 & 0.0237 & 0.0046 & 0.0026 & 0.0244 & 0.0054 & 0.0027 \\
\hline & 0.75 & 0.0136 & 0.0034 & 0.0020 & 0.0167 & 0.0033 & 0.0021 & 0.0165 & 0.0035 & 0.0020 \\
\hline \multirow{3}{*}{$g_{T 2}^{2}(z)$} & 0.25 & 0.3628 & 0.2612 & 0.2495 & 0.4173 & 0.2912 & 0.2650 & 0.4803 & 0.3501 & 0.2925 \\
\hline & 0.50 & 0.4046 & 0.3460 & 0.3186 & 0.4456 & 0.3602 & 0.3258 & 0.5399 & 0.4396 & 0.4333 \\
\hline & 0.75 & 0.5627 & 0.4743 & 0.4673 & 0.5719 & 0.5444 & 0.5221 & 0.7106 & 0.6611 & 0.6197 \\
\hline
\end{tabular}

$(z=0.25,0.5,0.75)$ as in setting (i) and calculate the estimated standard errors over 1000 replications of the following quantities:

$$
\begin{aligned}
& \mathrm{g}_{\mathrm{T} 1}^{2}(z)=\widetilde{\mathrm{p}}_{1}(z)\left[\widehat{\beta}_{1}(z)-\beta_{1}(z)\right]+\widetilde{\mathrm{p}}_{2}(z)\left[\widehat{\beta}_{2}(z)-\beta_{2}(z)\right], \\
& \mathrm{g}_{\mathrm{T} 2}^{2}(z)=\widetilde{\mathrm{p}}_{2}(z)\left[\widehat{\beta}_{1}(z)-\beta_{1}(z)\right]-\widetilde{\mathrm{p}}_{1}(z)\left[\widehat{\beta}_{2}(z)-\beta_{2}(z)\right] .
\end{aligned}
$$

In this simulation, we find that the choice of the bandwidth $h$ has only a small impact on the kernel estimation results in the direction of $\widetilde{q}_{\mathrm{T}}(z)$, but affects those in the direction of $\widetilde{\mathrm{q}}_{\mathrm{T}}^{\perp}(z)$ quite a lot. We use the bandwidth $h=c_{h} T^{-0.8}$ with $c_{h}=1.05$ in the kernel estimation. Such a bandwidth selection may seem somewhat arbitrary, but it leads to sensible results for the kernel estimation standard errors in Table 2. In particular, it is clear from the table that the values in both directions approach zero as the sample size increases, and $g_{\mathrm{T} 1}^{2}(z)$ evidently converges at a rate much faster than $\mathrm{g}_{\mathrm{T} 2}^{2}(z)$, as indicated in Section 3.1 where $g_{\mathrm{T} 1}^{2}(z)=\mathrm{O}_{\mathrm{P}}\left(\frac{1}{\mathrm{~T} \sqrt{\mathrm{Th}}}+\mathrm{h}^{\gamma}\right)$ and $g_{\mathrm{T} 2}^{2}(z)=\mathrm{O}_{\mathrm{P}}\left(\frac{1}{\mathrm{Th}}+\mathrm{h}^{\gamma}\right)$ for fixed $z \in(0,1)$. In addition, the choice of bandwidth also slows down the rate of convergence in the direction of $\widetilde{q}_{T}^{\perp}(z)$. As in setting (i), Table 2 shows that correlation within the nonstationary regressors $X_{t}$ has little impact on the results.

(iii) Following (i) we let $d=3$ and consider the data generating process (6.1) with $X_{t}=\left(X_{t 1}, X_{t 2}^{\prime}\right)^{\prime}$, where

$$
\Delta \mathrm{X}_{\mathrm{t} 1}=\mathrm{e}_{\mathrm{t} 1} \text { and } \mathrm{X}_{\mathrm{t} 2}=\left(\begin{array}{c}
0.05 \\
0.15
\end{array}\right)+\left(\begin{array}{cc}
0.5 & 0.3 \\
0 & 1
\end{array}\right) \mathrm{X}_{\mathrm{t}-1,2}+\left(\begin{array}{c}
e_{\mathrm{t} 2} \\
e_{\mathrm{t} 3}
\end{array}\right)
$$

It can be shown that $X_{t 2}$ is cointegrated with a linear deterministic trend. The processes $e_{t}=\left(e_{t 1}, e_{t 2}, e_{t 3}\right)^{\prime}$ and $e_{t 0}$ are defined as in setting (i), and the coefficient functions $\beta_{t}=\beta(t / T)=\left(\beta_{t 1}, \beta_{t 2}, \beta_{t 3}\right)^{\prime}$ are defined in the same way as (i) with three different functions.

This design is intended to assess the limit theory in Section 3.2 where the nonstationary regressors are cointegrated with deterministic trends. The global rotation matrix is $\mathbf{H}=\left(\mathbf{H}_{1}, \mathbf{H}_{2}\right)$, where

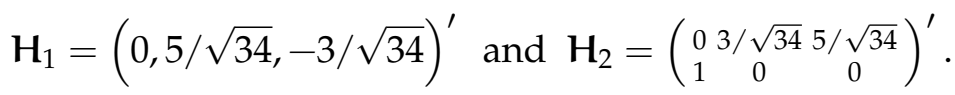

The local rotation matrix $\overline{\mathbf{Q}}_{\mathrm{T}}(z)$ is the same as that in setting (i) with $\mathbf{H}_{2}^{\prime} \mathrm{X}_{\mathrm{t}}$ replacing $\mathrm{X}_{\mathrm{t} 2}$. The kernel estimates in three different directions (as discussed in Section 3.2) are

$$
\mathbf{H}_{1}^{\prime} \widehat{\beta}(z), \quad \mathrm{q}_{\mathrm{T} 2}(z)^{\prime} \mathbf{H}_{2}^{\prime} \widehat{\beta}(z) \text { and } \mathrm{q}_{\mathrm{T} 2}^{\perp}(z)^{\prime} \mathbf{H}_{2}^{\prime} \widehat{\beta}(z),
$$


Table 3: Standard errors of kernel estimates in three different directions for setting (iii)

\begin{tabular}{|c|c|c|c|c|c|c|c|c|c|c|}
\hline \multirow[b]{2}{*}{$n$} & \multirow[t]{2}{*}{$z$} & \multicolumn{3}{|c|}{$\rho=0$} & \multicolumn{3}{|c|}{$\rho=0.2$} & \multicolumn{3}{|c|}{$\rho=0.5$} \\
\hline & & 200 & 600 & 1000 & 200 & 600 & 1000 & 200 & 600 & 1000 \\
\hline \multirow{3}{*}{$g_{\mathrm{T} 1}^{3}(z)$} & 0.25 & 0.3452 & 0.2706 & 0.2401 & 0.4086 & 0.3085 & 0.2739 & 0.4813 & 0.3704 & 0.3264 \\
\hline & 0.50 & 0.3672 & 0.2993 & 0.2665 & 0.4107 & 0.3288 & 0.2977 & 0.5561 & 0.3925 & 0.3416 \\
\hline & 0.75 & 0.4130 & 0.3910 & 0.3541 & 0.4755 & 0.4171 & 0.3699 & 0.6056 & 0.4907 & 0.4578 \\
\hline \multirow{3}{*}{$g_{\mathrm{T} 2}^{3}(z)$} & 0.25 & 0.0518 & 0.0158 & 0.0084 & 0.0555 & 0.0135 & 0.0068 & 0.0522 & 0.0141 & 0.0062 \\
\hline & 0.50 & 0.0243 & 0.0063 & 0.0035 & 0.0244 & 0.0075 & 0.0034 & 0.0273 & 0.0061 & 0.0033 \\
\hline & 0.75 & 0.0191 & 0.0052 & 0.0026 & 0.0173 & 0.0047 & 0.0027 & 0.0212 & 0.0047 & 0.0027 \\
\hline \multirow{3}{*}{$g_{\mathrm{T} 3}^{3}(z)$} & 0.25 & 0.1629 & 0.0945 & 0.0613 & 0.1853 & 0.0853 & 0.0718 & 0.2071 & 0.0993 & 0.0682 \\
\hline & 0.50 & 0.2933 & 0.2324 & 0.2204 & 0.3012 & 0.2419 & 0.2189 & 0.3607 & 0.2602 & 0.2314 \\
\hline & 0.75 & 0.3999 & 0.3573 & 0.3385 & 0.3965 & 0.3660 & 0.3605 & 0.4570 & 0.4000 & 0.3904 \\
\hline
\end{tabular}

respectively. As in settings (i) and (ii), we consider three interior points: $z=0.25,0.5,0.75$, and then calculate the standard errors over 1000 replications of the following quantities:

$$
\begin{aligned}
& g_{\mathrm{T} 1}^{3}(z)=5 / \sqrt{34}\left(\widehat{\beta}_{2}(z)-\beta_{2}(z)\right)-3 / \sqrt{34}\left(\widehat{\beta}_{3}(z)-\beta_{3}(z)\right), \\
& g_{\mathrm{T} 2}^{3}(z)=p_{1}(z)\left[3 / \sqrt{34}\left(\widehat{\beta}_{2}(z)-\beta_{2}(z)\right)+5 / \sqrt{34}\left(\widehat{\beta}_{3}(z)-\beta_{3}(z)\right)\right]+p_{2}(z)\left[\widehat{\beta}_{1}(z)-\beta_{1}(z)\right], \\
& g_{3 \mathrm{~T}}^{3}(z)=p_{2}(z)\left[3 / \sqrt{34}\left(\widehat{\beta}_{2}(z)-\beta_{2}(z)\right)+5 / \sqrt{34}\left(\widehat{\beta}_{3}(z)-\beta_{3}(z)\right)\right]-p_{1}(z)\left[\widehat{\beta}_{1}(z)-\beta_{1}(z)\right] .
\end{aligned}
$$

Unlike settings (i) and (ii), we use different bandwidths when computing the kernel estimation standard errors in different directions. From Table 3, all estimated standard errors decrease as the sample size increases. Broadly speaking, the values of $g_{\mathrm{T} 2}^{3}(z)$ converge to zero much faster than $\mathrm{g}_{\mathrm{T} 1}^{3}(z)$ and $\mathrm{g}_{\mathrm{T} 3}^{3}(z)$, and $g_{\mathrm{T} 1}^{3}(z)$ looks to have the largest value, supporting the limit results in Section 3.2. In addition, the presence of the correlation between the stationary component $X_{t 1}$ and the nonstationary component $X_{t 2}$ still has no impact on the kernel estimation results.

(iv) In the above three settings, the error $e_{t 0}$ is independent of the nonstationary regressors, excluding the existence of endogeneity. We now relax this restriction when generating the simulated data. As shown in Section 2.3, the correlation between $e_{\mathrm{t} 0}$ and the stationary regressors often leads to estimation inconsistency. We now consider the setting (i) with the only difference that $e_{t 0}$ and $\left(e_{t 2}, e_{t 3}\right)^{\prime}$ are jointly determined by a three-dimensional normal distribution with mean zero and the covariance matrix:

$$
\Omega_{3}=\left(\begin{array}{ccc}
1 & 0.5 & 0.5 \\
0.5 & 1 & 0.5 \\
0.5 & 0.5 & 1
\end{array}\right)
$$

and $e_{\mathrm{t} 1}$ is independent of $\left(e_{\mathrm{t} 0}, e_{\mathrm{t} 2}, e_{\mathrm{t} 3}\right)^{\prime}$.

We compare finite sample performance between the conventional kernel estimates and the FM kernel estimates proposed in Section 4 . We use the same interior points $(z=0.25,0.5,0.75)$ and calculate averages 
Table 4: Averages of $g_{T i}^{\#}(z)$ and $g_{T i}^{4}(z)$ over 1000 replications for setting (iv)

\begin{tabular}{|c|c|c|c|c|c|c|c|c|c|}
\hline \multirow[b]{2}{*}{$\mathrm{T}$} & \multirow[t]{2}{*}{$z$} & \multicolumn{3}{|c|}{ FM-kernel } & & \multirow[t]{2}{*}{$z$} & \multicolumn{3}{|c|}{ Kernel } \\
\hline & & 200 & 600 & 1000 & & & 200 & 600 & 1000 \\
\hline \multirow{3}{*}{$g_{\mathrm{T} 1}^{\#}(z)$} & 0.25 & 0.01368 & 0.00401 & 0.00002 & \multirow{3}{*}{$\mathrm{g}_{\mathrm{T} 1}^{4}(z)$} & 0.25 & 0.01229 & 0.00198 & -0.00009 \\
\hline & 0.50 & 0.00687 & 0.00477 & 0.00443 & & 0.50 & 0.00803 & 0.00550 & 0.00429 \\
\hline & 0.75 & -0.01006 & -0.00884 & 0.00536 & & 0.75 & -0.01451 & -0.00938 & 0.00507 \\
\hline \multirow{3}{*}{$g_{\mathrm{T} 2}^{\#}(z)$} & 0.25 & 0.00188 & 0.00073 & 0.00065 & \multirow{3}{*}{$g_{\mathrm{T} 2}^{4}(z)$} & 0.25 & -0.00053 & 0.00037 & 0.00085 \\
\hline & 0.50 & 0.00042 & 0.00070 & 0.00056 & & 0.50 & 0.00024 & 0.00062 & -0.00060 \\
\hline & 0.75 & 0.00044 & 0.00033 & 0.00037 & & 0.75 & -0.00051 & 0.00036 & -0.00044 \\
\hline \multirow{3}{*}{$g_{\mathrm{T} 3}^{\#}(z)$} & 0.25 & -0.00573 & -0.00343 & -0.00184 & \multirow{3}{*}{$g_{\mathrm{T} 3}^{4}(z)$} & 0.25 & -0.00526 & -0.00523 & 0.00298 \\
\hline & 0.50 & -0.00345 & -0.00198 & 0.00050 & & 0.50 & -0.00132 & -0.00323 & 0.00173 \\
\hline & 0.75 & 0.00116 & 0.00302 & 0.00051 & & 0.75 & -0.00180 & 0.00358 & 0.00092 \\
\hline
\end{tabular}

of the following quantities over 1000 replications

$$
\begin{aligned}
& g_{\mathrm{T} 1}^{\#}(z)=\widehat{\beta}_{1}^{\#}(z)-\beta_{1}(z), \\
& g_{\mathrm{T} 2}^{\#}(z)=p_{1}(z)\left[\widehat{\beta}_{2}^{\#}(z)-\beta_{2}(z)\right]+p_{2}(z)\left[\widehat{\beta}_{3}^{\#}(z)-\beta_{3}(z)\right], \\
& g_{\mathrm{T} 3}^{\#}(z)=p_{2}(z)\left[\widehat{\beta}_{2}^{\#}(z)-\beta_{2}(z)\right]-p_{1}(z)\left[\widehat{\beta}_{3}^{\#}(z)-\beta_{3}(z)\right],
\end{aligned}
$$

where $\widehat{\boldsymbol{\beta}}_{\#}(z)=\left[\widehat{\beta}_{1}^{\#}(z), \widehat{\beta}_{2}^{\#}(z), \widehat{\beta}_{3}^{\#}(z)\right]^{\prime}$ is defined in (4.8). The estimate of the long run covariance matrix is constructed under $l_{T}=\left\lfloor T^{1 / 4}\right\rfloor, \tau=1 / 4$, and $k(x)=I(-1 \leqslant x \leqslant 1)$. Accordingly, we define $g_{T i}^{4}(z)$ in a similar way as $g_{T i}^{\#}(z)$, with $\widehat{\beta}_{i}^{\#}(z)$ being replaced by the conventional kernel estimate $\widehat{\beta}_{i}(z)$ for $i=1,2,3$.

The simulation results are reported in Table 4 for both the FM and conventional kernel methods. The methods exhibit similar finite sample performance in the three directions. The results show that the FMkernel method improves estimation well when $T=1000$, but has similar performance to the conventional kernel estimation when the sample size is small or moderate. Broadly speaking, the simulation shows that reasonably large samples are needed to realize gains from the use of FM kernel methods in the present setting. These findings generally support the limit theory in Section 4.

EXAMPLE 6.2. We use the data generating process

$$
Y_{t}=\beta_{t}^{\prime} X_{t}+e_{t 0}, \quad \beta_{t} \equiv \beta_{0}=(2,2,4)^{\prime}, \quad t=1, \cdots, T,
$$

where $X_{t}=\left(X_{t 1}^{\prime}, X_{t 2}\right)^{\prime}, X_{t 1}=\left(e_{t 1}, e_{t 2}\right)^{\prime}, \Delta X_{t 2}=e_{t 3}, e_{t}=\left(e_{t 1}, e_{t 2}, e_{t 3}\right)^{\prime}$ and $e_{t 0}$ are defined as in setting (i) with $\rho=0$ or 0.2 . This simulation is designed to examine the size performance of the nonparametric test statistics proposed in Section 5.

Consider the global null hypothesis:

$$
\mathcal{H}_{0}: \mathbf{R}\left(\boldsymbol{\beta}_{\mathrm{t}}-\boldsymbol{\beta}_{0}\right)=\mathbf{0}_{2}, \quad \mathbf{R}=\left(\begin{array}{ccc}
1 & 0 & 0 \\
0 & 1 & 0
\end{array}\right),
$$

which is equivalent to testing $\beta_{\mathrm{t} 1}=\beta_{\mathrm{t} 2} \equiv 2$, given the true value $\beta_{0}$. In this case, it is easy to verify the rank condition (5.5) with $r=2$. So we can use the generalized Wald test statistic constructed in (5.4) and (5.7), and examine test size performance in finite samples. 
Table 5: Empirical size performance under the global null hypothesis

\begin{tabular}{cccccccc}
\hline \hline \multirow{2}{*}{$\mathrm{T}$} & \multicolumn{3}{c}{$\rho=0$} & & \multicolumn{3}{c}{$\rho=0.2$} \\
\cline { 2 - 4 } \cline { 6 - 8 } & $1 \%$ & $5 \%$ & $10 \%$ & & $1 \%$ & $5 \%$ & $10 \%$ \\
\hline 200 & 0.0178 & 0.0588 & 0.0936 & & 0.0188 & 0.0568 & 0.0922 \\
400 & 0.0134 & 0.0520 & 0.0957 & & 0.0141 & 0.0527 & 0.0935 \\
1000 & 0.0108 & 0.0510 & 0.0982 & & 0.0101 & 0.0504 & 0.0976 \\
\hline \hline
\end{tabular}

Table 6: Empirical size performance under the local null hypothesis

\begin{tabular}{cccccccc}
\hline \hline \multirow{2}{*}{$\mathrm{T}$} & \multicolumn{3}{c}{$\rho=0$} & & \multicolumn{3}{c}{$\rho=0.2$} \\
\cline { 2 - 4 } \cline { 6 - 8 } & $1 \%$ & $5 \%$ & $10 \%$ & & $1 \%$ & $5 \%$ & $10 \%$ \\
\hline 200 & 0.0149 & 0.0523 & 0.0946 & & 0.0168 & 0.0584 & 0.0946 \\
400 & 0.0118 & 0.0514 & 0.0949 & & 0.0148 & 0.0549 & 0.0978 \\
1000 & 0.0105 & 0.0504 & 0.0989 & & 0.0100 & 0.0507 & 0.0987 \\
\hline \hline
\end{tabular}

As in (5.10), we also construct the following local null hypothesis:

$$
\mathcal{H}_{0}^{\diamond}: \mathbf{R}(z)\left(\beta_{\mathrm{t}}-\beta_{0}\right)=\mathbf{0}_{2}, \quad \mathbf{R}(z)=\left(\begin{array}{ccc}
1 & -1 & 0 \\
0 & 0 & \mathrm{q}_{\mathrm{T}}(z)
\end{array}\right), \mathrm{q}_{\mathrm{T}}(z)=X_{\lfloor\mathrm{T}(z-\mathrm{h})\rfloor, 2} /\left|\mathrm{X}_{\lfloor\mathrm{T}(z-\mathrm{h})\rfloor, 2}\right| .
$$

In this case, the rank condition (5.5) fails, so we adopt the generalized Wald test statistic defined in (5.13) and (5.14) and examine its size performance.

A bootstrap scheme is used to generate the critical values $l_{\alpha}^{*}$ where $\alpha=1 \%, 5 \%$, and $10 \%$, as it is well known that bootstrap critical values usually outperform the asymptotic critical values in finite samples. A detailed description of the bootstrap procedure is provided in Appendix $C$ of the Online Supplement (Li, Phillips and Gao, 2019). The sample sizes considered are $T=200,400$, and 1000, and the number of Monte-Carlo replications is $M=1000$. The size function is defined by

$$
\alpha_{\mathrm{T}}^{*}=\mathrm{P}\left(\mathrm{W}_{\mathrm{T}} \geqslant l_{\alpha}^{*} \mid \mathcal{H}_{0}\right) \text { or } \alpha_{\mathrm{T}}^{*}=\mathrm{P}\left(\mathrm{W}_{\mathrm{T}}^{\diamond} \geqslant l_{\alpha}^{*} \mid \mathcal{H}_{0}^{\diamond}\right),
$$

where $W_{T}$ is defined in (5.7) and $W_{\mathrm{T}}^{\diamond}$ is defined in (5.14). As the size function defined in (6.6) is also a function of the significance level $\alpha$, different bandwidths $\left\{h_{i}: i=1,2,3\right\}$ are used in simulations with respect to $\alpha=1 \%, 5 \%$, and $10 \%$, respectively. We use a grid search method to find appropriate bandwidths that lead to good size performance.

The simulation results of the empirical size for both the global and local null hypothesis tests are summarized in Tables 5 and 6 . We observe that almost all the calculated sizes fluctuate around the given significance level. They appear to be oversized at the $1 \%$ and $5 \%$ significance level and undersized at $10 \%$ for both hypothesis tests, but these size distortions decrease as the sample size increases. The presence of the correlation between the stationary component $X_{t 1}$ and the nonstationary component $X_{t 2}$ again has no noticeable impact on size performance. In addition, we study the finite-sample performance of the proposed test under an alternative hypothesis and the power results are found to be satisfactory for small-moderate departures even for sample sizes $T=200,400$. The details are reported in Appendix $C$ of the Online Supplement. 


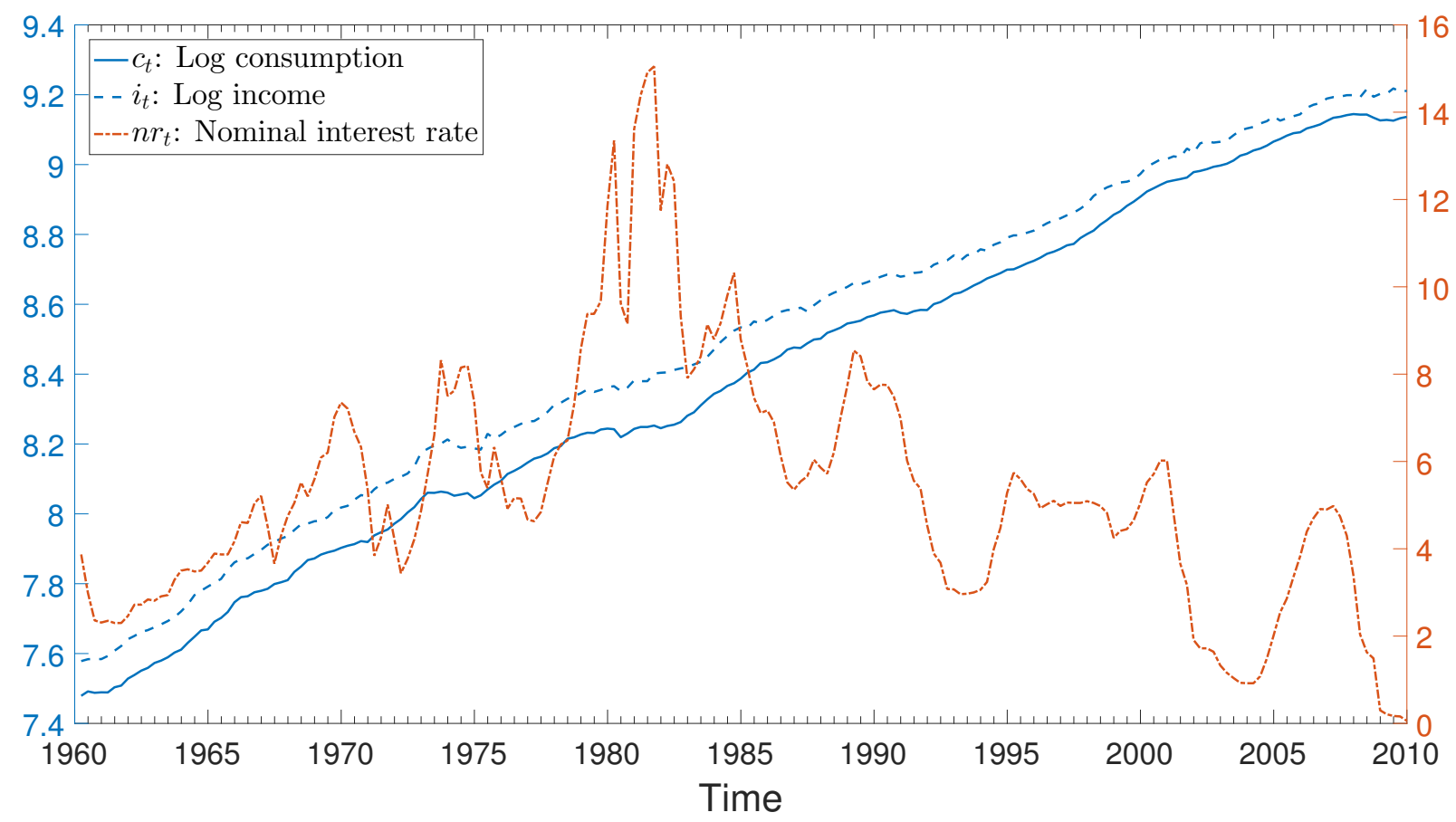

Figure 1: Aggregate US data on consumption, income and nominal interest rate over 1960-2009.

\section{Empirics: time-varying consumption behaviour}

We next apply the time-varying coefficient model and kernel estimation methodology to aggregate US data on consumption, income, and nominal interest rate obtained from Federal Reserve Economic Data (FRED). We consider a quarterly data set over the first quarter of 1960 to the last quarter of 2009 with 200 observations: $c_{t}$ is log-transformed consumption expenditure, $i_{t}$ is log-transformed disposable income, and $n r_{t}$ is the nominal interest rate expressed as a percentage. All the three series are plotted in Figure 1, which shows that $c_{t}$ and $i_{t}$ have co-moving trend components. Unit root tests confirm nonstationarity for all variables. ${ }^{1}$

Set $Y_{t}=c_{t}$ and $X_{t}=\left(i_{t}, i_{t-1}, n r_{t}\right)^{\prime}$, where $i_{t}$ and $i_{t-1}$ are cointegrated regressors, and $n r_{t}$ follows a unit root process. Noting that $i_{t}-i_{t-1}$ is stationary, as in Section 2.1 we may apply the global transformation matrix $\mathbf{H}=\left(\mathbf{H}_{1}, \mathbf{H}_{2}\right)$ with

$$
\mathbf{H}_{1}=(\sqrt{2} / 2,-\sqrt{2} / 2,0)^{\prime} \text { and } \mathbf{H}_{2}=\left(\begin{array}{ccc}
\sqrt{2} / 2 & \sqrt{2} / 2 & 0 \\
0 & 0 & 1
\end{array}\right)^{\prime},
$$

on the covariate space to separate out stationary and nonstationary components as $X_{t 1}=H_{1}^{\prime} X_{t}$ and $X_{t 2}=H_{2}^{\prime} X_{t}$, respectively. We first fit the following time-varying coefficient model:

$$
Y_{t}=\beta_{t}^{\prime} \bar{X}_{t}+e_{t}, \beta_{t}=\beta(t / T), t=1, \cdots, T,
$$

where $\bar{X}_{t}=\mathbf{H}^{\prime} X_{t}=\left(X_{t 1}, X_{t 2}^{\prime}\right)^{\prime}$ and $T=200$. For given $0<\tau<1$, the coefficient function $\beta(\tau)=$

\footnotetext{
${ }^{1}$ The PP tests (Phillips and Perron, 1988) with fitted mean and linear trend were conducted for $c_{t}$ and $i_{t}$, giving $p$-values of 0.7248 and 0.7603 . The PP test with fitted mean for $n r_{t}$ gave a $p$-value of 0.2661 .
} 


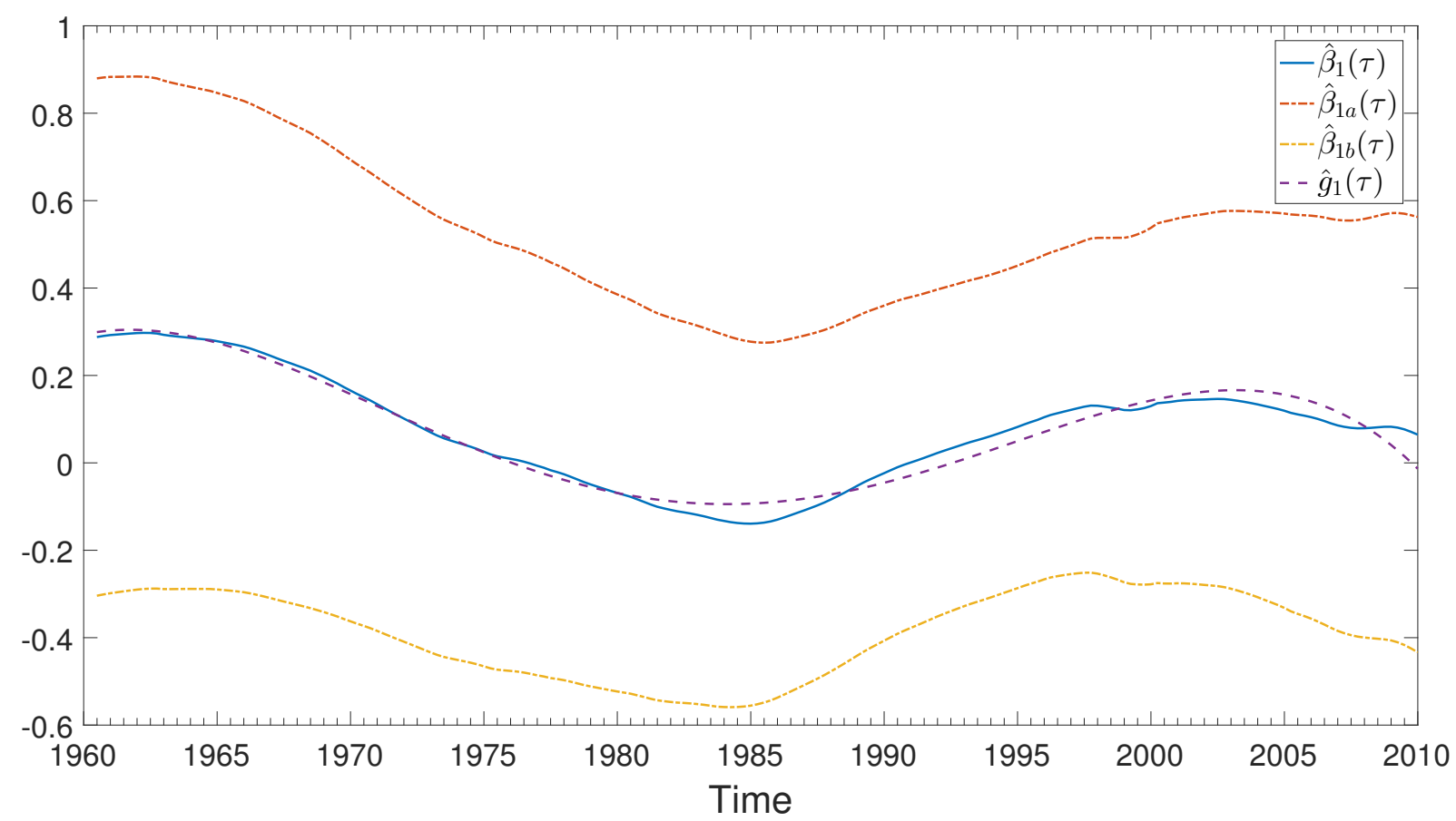

Figure 2: Nonparametric function estimate $\widehat{\beta}_{1}$ with confidence intervals $\left(\widehat{\beta}_{1 \mathrm{a}}, \widehat{\beta}_{1 \mathrm{~b}}\right)$ together with the $4^{\text {th }}$ order parametric polynomial $\widehat{g}_{1}$ estimate of $\beta_{1}$.

$\left[\beta_{1}(\tau), \beta_{2}(\tau), \beta_{3}(\tau)\right]^{\prime}$ is estimated by the local level regression as

$$
\widehat{\boldsymbol{\beta}}(\tau)=\left[\widehat{\beta}_{1}(\tau), \widehat{\beta}_{2}(\tau), \widehat{\beta}_{3}(\tau)\right]^{\prime}=\left[\sum_{t=1}^{T} \bar{X}_{t} \bar{X}_{t}^{\prime} K\left(\frac{t-T \tau}{T h}\right)\right]^{+}\left[\sum_{t=1}^{T} \bar{X}_{t} Y_{t} K\left(\frac{t-T \tau}{T h}\right)\right],
$$

where $\mathrm{K}(\mathrm{u})=\frac{3}{4}\left(1-\mathrm{u}^{2}\right) \mathrm{I}(-1 \leqslant u \leqslant 1)$ and the bandwidth $\mathrm{h}=\mathrm{c} \cdot \mathrm{h}_{\mathrm{c} v}=15 \times \mathrm{h}_{\mathrm{cv}}=0.4990$ with $\mathrm{h}_{\mathrm{c} v}$ being the CV selected bandwidth. The three nonparametrically estimated curves $\widehat{\beta}_{i}(\cdot)$ with their $95 \%$ confidence intervals are exhibited in Figures 2-4, where the confidence intervals are computed using the bootstrap approach.

For comparison, we also consider a traditional linear consumption function of the following form

$$
Y_{t}=\beta_{\star}^{\prime} \bar{X}_{t}+v_{t}, \beta_{\star}=\left(\beta_{1}^{\star}, \beta_{2}^{\star}, \beta_{3}^{\star}\right)^{\prime},
$$

whose constant coefficients are estimated as $\widehat{\boldsymbol{\beta}}_{\star}=(-0.4099,0.7019,-0.0065)^{\prime}$. The constant coefficient specification (7.3) fails to capture any time-varying components in the coefficients, whereas plots of the fitted functions $\widehat{\beta}_{1}(\cdot), \widehat{\beta}_{2}(\cdot)$ and $\widehat{\beta}_{3}(\cdot)$ in Figures $2-4$ strongly support the presence of nonlinear functional forms for these coefficients. Based on the observed patterns of $\widehat{\beta}_{j}(\cdot)$, a high-order polynomial function might be a good parametric candidate for approximating the estimated time-varying coefficient functions. Accordingly, we fitted $4^{\text {th }}$ order polynomial functions for each of the coefficient functions. The plots of these fitted polynomial functions ${ }^{2}$ are shown in Figures 2-4. Standard t-tests were used to select the chosen specifications of the polynomial functions and, although not detailed here, the coefficients in the selected specifications were significant with p-values close to zero. Figures $2-4$ show that the nonparametric fits

\footnotetext{
${ }^{2}$ The fitted functions are $\widehat{\mathrm{g}}_{1}(\tau)=0.2968+0.5368 \tau-9.8343 \tau^{2}+19.8220 \tau^{3}-10.8344 \tau^{4}, \widehat{\mathrm{g}}_{2}(\tau)=0.6983-0.0120 \tau+0.0715 \tau^{2}-$ $0.0858 \tau^{3}+0.0303 \tau^{4}, \widehat{g}_{3}(\tau)=-0.0028+0.0150 \tau-0.0763 \tau^{2}+0.0762 \tau^{3}-0.0159 \tau^{4}$.
} 


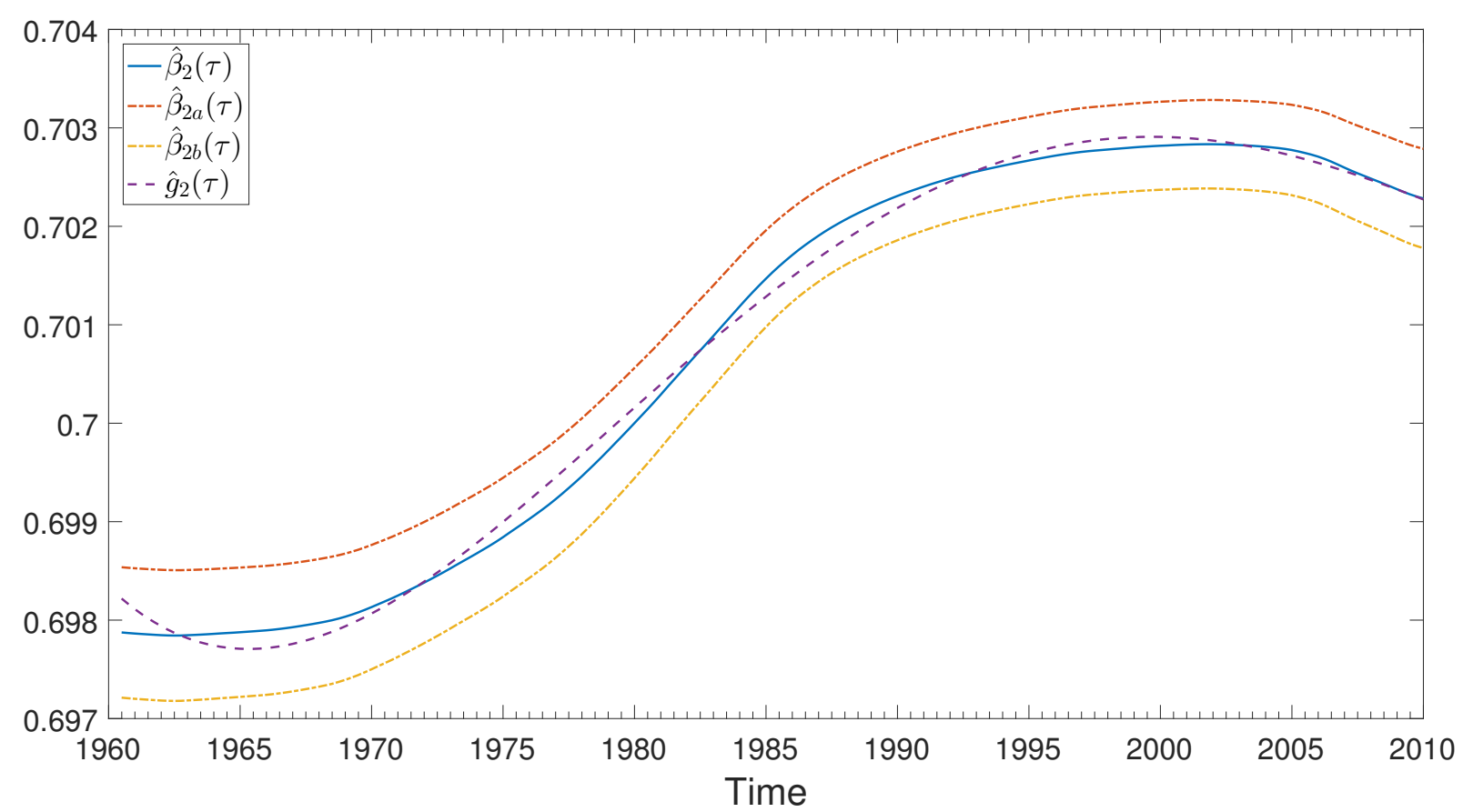

Figure 3: Nonparametric function estimate $\widehat{\beta}_{2}$ with confidence intervals $\left(\widehat{\beta}_{2 a}, \widehat{\beta}_{2 b}\right)$ together with the $4^{\text {th }}$ order parametric polynomial $\widehat{g}_{2}$ estimate of $\beta_{2}$.

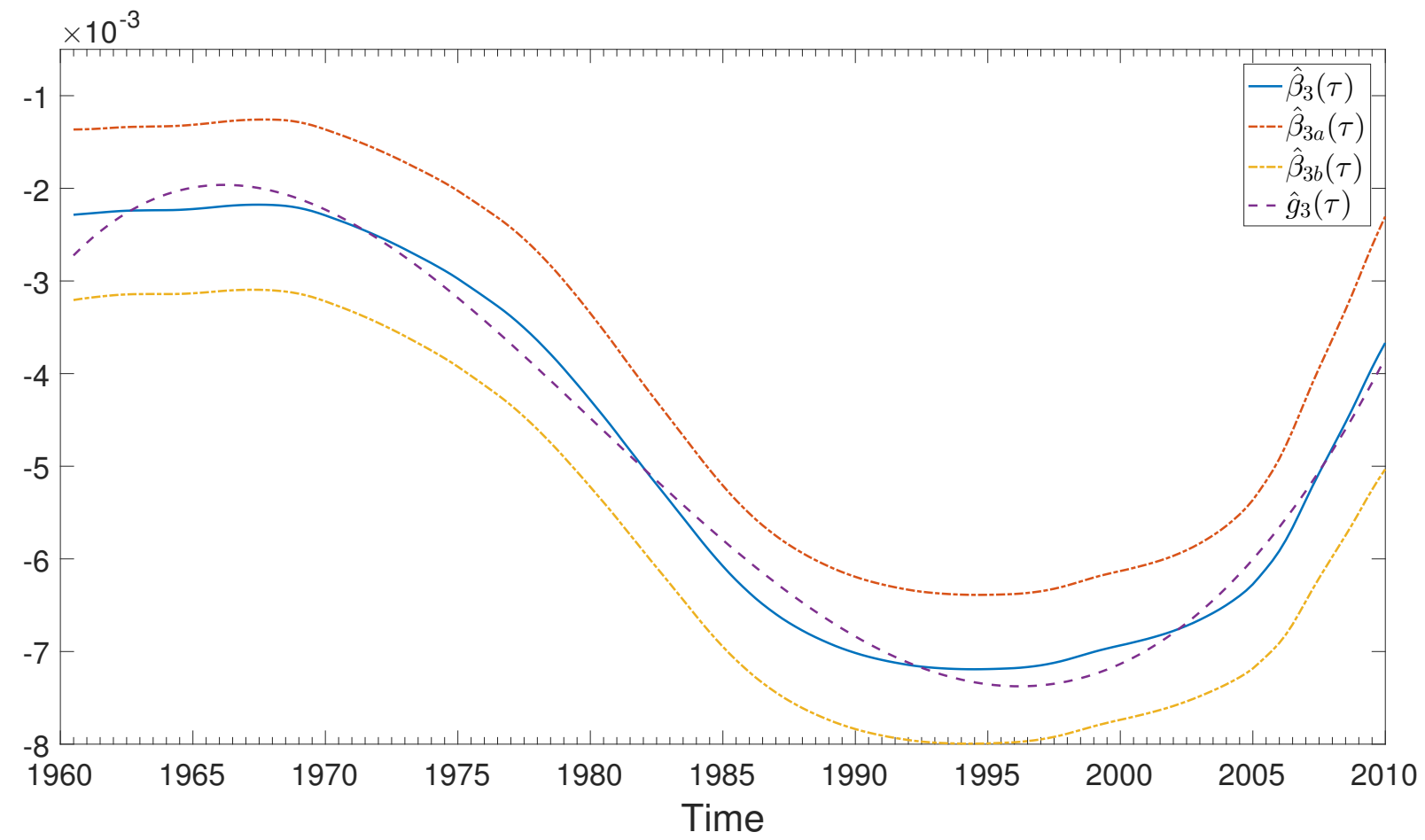

Figure 4: Nonparametric function estimate $\widehat{\beta}_{3}$ with confidence intervals $\left(\widehat{\beta}_{3 a}, \widehat{\beta}_{3 b}\right)$ together with the $4^{\text {th }}$ order parametric polynomial $\widehat{g}_{3}$ estimate of $\beta_{3}$. 
Table 7: Unit root tests for the residuals

\begin{tabular}{ccccc}
\hline \hline & ADF & DF-GLS & PP & KPSS \\
\hline$\widehat{e}_{\mathrm{t}}$ & $-3.85^{* * *}$ & $-2.82^{* * *}$ & $-4.03^{* * *}$ & 0.33 \\
$\widehat{v}_{\mathrm{t}}$ & $-2.99^{* *}$ & $-2.74^{* * *}$ & $-2.85^{*}$ & $1.36^{* * *}$ \\
\hline \hline
\end{tabular}

$*, * *$, and ${ }^{* * *}$ imply rejection of the null hypothesis at $10 \%, 5 \%$, and $1 \%$ level.

are well captured by the $4^{\text {th }}$ order parametric polynomial approximations with no need for higher order specifications.

Proceeding further, we analyzed residuals from the time-varying coefficient model (7.1) and the linear model (7.3), which are plotted in Figure S.1 available in Appendix C of the supplementary document. The residuals $\widehat{v}_{t}$ from the linear consumption function show a clear upward drift when compared with the residuals $\widehat{e}_{t}$ of the time-varying coefficient model. Standard residual based unit root tests ${ }^{3}$, shown in Table 7 , indicate stronger evidence for stationarity in $\widehat{e}_{t}$ than $\widehat{v}_{t}$. For example, when the PP test is applied, the null hypothesis is rejected at the $1 \%$ level for $\widehat{e}_{t}$ but the null fails to be rejected at the $5 \%$ level for $\widehat{v}_{t}$. In addition, the KPSS test suggests that $\widehat{v}_{t}$ may have a unit root at the $1 \%$ level. Based on these results, we conclude that $\widehat{e}_{t}$ is stationary, but $\widehat{v}_{t}$ is nonstationary, indicating that a time-varying coefficient consumption function is more appropriate in capturing cointegrating links between the variables than a linear model for consumption behavior.

In order to capture the drift presented in $\widehat{v}_{t}$, we fitted a fixed design nonparametric specification $\widehat{v}_{t}=m(t / T)+u_{t}, t=1, \cdots, T$, to the residuals using local level kernel estimation

$$
\widehat{m}(\tau)=\left(\sum_{t=1}^{T} K\left(\frac{t-T \tau}{T h}\right) \widehat{v}_{t}\right) /\left(\sum_{t=1}^{T} K\left(\frac{t-T \tau}{T h}\right)\right), 0<\tau<1 .
$$

to estimate the trend function $m(\cdot)$. The estimated trend is shown in Figure 5, which is strongly indicative of a nonlinear trend in $\widehat{v}_{t}$. The detrended residuals $\widehat{u}_{t}:=\widehat{v}_{t}-\widehat{m}(t / T)$ from this nonparametric regression are plotted in Figure S.2 (available in Appendix C of the supplementary document) against the residuals $\widehat{e}_{t}$ from the time varying coefficient consumption function. The close correspondence of these residuals provides further confirmation of the presence of time variation in the consumption function.

\section{Conclusions}

Nonparametric methods offer empirical researchers considerable flexibility in model specifications, allowing for time dependent formulations that are useful when models with constant coefficients prove inadequate. In time series regressions, this flexibility is particularly useful when series move together over time but fail cointegration tests because of evolving coefficients. The kernel estimation approach studied in the present paper allows empirical research with time-varying coefficient cointegrating models when the regressors are multivariate and embody a mixture of stochastic and deterministic trends combined with potential co-movement among themselves. This structure is sufficiently rich to accommodate many empirical

\footnotetext{
${ }^{3}$ Formal residual based unit root tests (e.g., Phillips and Ouliaris, 1990) are unavailable for specifically testing residuals from time varying coefficient cointegrating regressions and are presently under development by the authors in a separate project. Standard unit root tests are used here instead.
} 


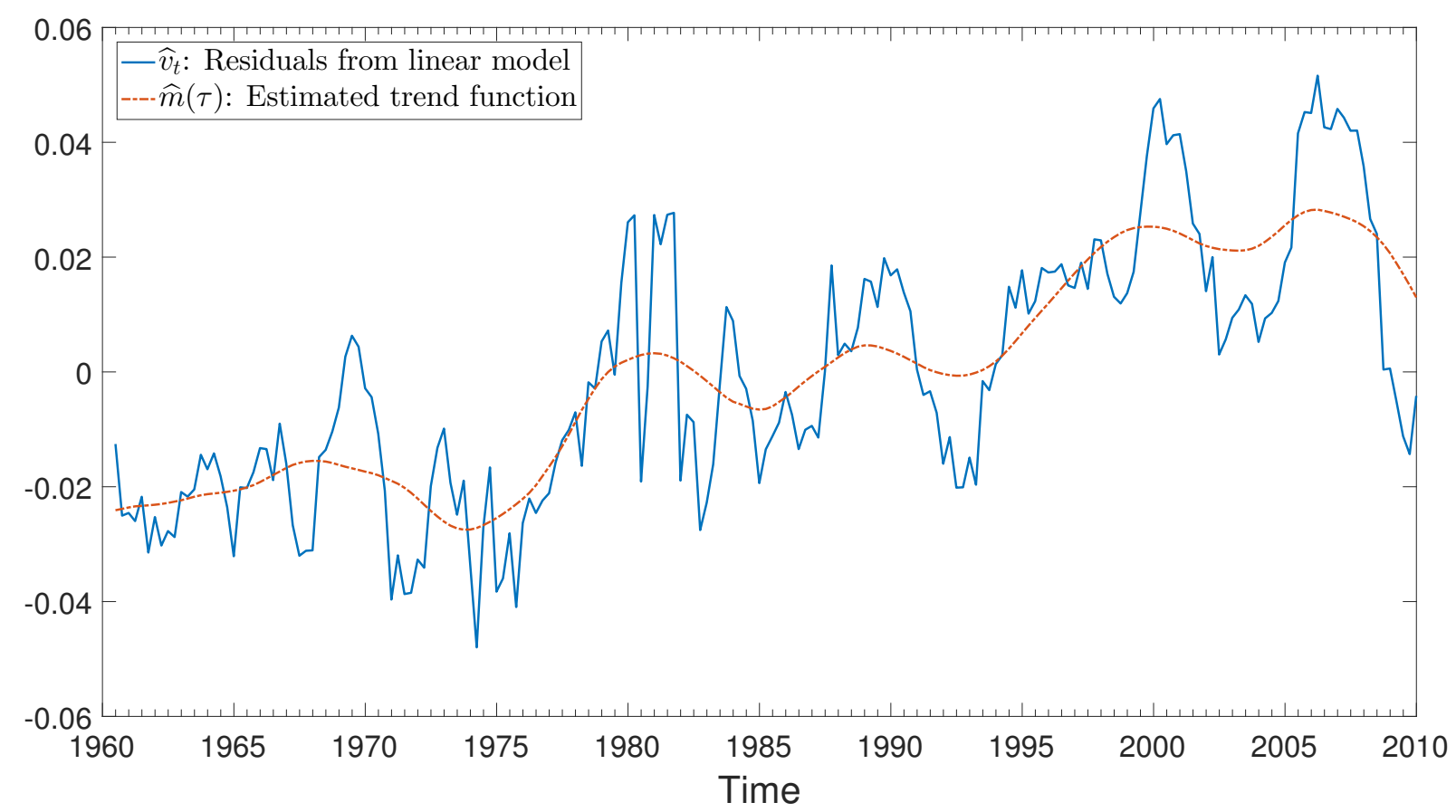

Figure 5: The estimated nonlinear trend of the residuals $\widehat{v}_{t}$ from the linear model

applications with co-moving nonstationary time series. Standard local level kernel regression forms the basis of the approach and the FM-kernel methodology extends to nonparametric regression the FM-OLS method of estimating linear cointegrating regressions with endogenous regressors and serially dependent error processes.

The methods are straightforward to implement and have the advantage that conventional limit theory can be used in a way that facilitates inference, even though the model complexities imply signal matrix degeneracies that lead to multiple convergence rates in different directions of the parameter space. In particular, the usual kernel convergence rate $(\sqrt{T h})$ applies in the stationary direction, a type 1 superconsistency rate $(T \sqrt{h})$ and a type 2 super-consistency rate $(T h)$ apply in nonstationary directions, and a type 3 rate $(T \sqrt{T h})$ applies in the direction of the deterministic linear trends. The local and global rotation techniques used in the paper to address these challenges are a technical device only. While they produce new asymptotic theory for kernel estimation techniques that differs considerably from standard kernel limit theory, the rotation methods are not needed in empirical research with these kernel estimators or with the test statistics that are based on them.

In addition to the estimation methodology and new limit theory for time-varying parameter cointegrating regression, a generalized Wald-type statistic is introduced to provide a statistical test of whether the timevarying coefficients can be approximated by constant coefficients. That methodology also allows for testing the adequacy of specific functional forms such as polynomial time-varying parameter specifications. These specification tests enable researchers to evaluate whether greater flexibility is needed in the formulation of cointegration regression models to allow for the coefficients in these models to evolve over time. Empirical application of these methods to aggregate consumption behavior in the US is strongly indicative of the need for such flexibility. In addition, the comprehensive simulation results support the limit results established in Sections 2-5.

The present work suggests future research on related matters of importance for practical implementation 
of the methods of this study. For example, as is apparent in the numerical simulations, it will be useful to develop a fully data-driven approach to bandwidth selection for time-varying cointegrating coefficient estimation by kernel methods and to establish its asymptotic validity. Another topic of importance is the development of residual based unit root and KPSS tests for time-varying coefficient cointegrating regressions and derive their asymptotic properties under null and alternative hypotheses.

\section{Acknowledgements}

The authors thank the Co-Editor, Oliver Linton, an Associate Editor and two reviewers for helpful comments, which have improved the earlier version of the paper. Phillips acknowledges NSF support under Grant Number: SES 12-85258 and the Kelly Fund at the University of Auckland. Gao acknowledges ARC Discovery Grant support under Grant Numbers: DP150101012 and DP170104421. Computing assistance by Weilun Zhou is also acknowledged and much appreciated.

\section{Supplement}

Readers are referred to the Online Supplement accompanying this paper for proofs of the main asymptotic theorems, some additional theory, and further numerical results.

\section{References}

Baek, Y., Cho, J. and Phillips, P. C. B. (2015). Testing linearity using power transforms of regressors. Journal of Econometrics 187, 376-384.

Billingsley, P. (1968). Convergence of Probability Measure, Wiley, New York.

Cai, Z. (2007). Trending time-varying coefficient time series models with serially correlated errors. Journal of Econometrics 136, $163-188$

Cai, Z., Li, Q. and Park, J. Y. (2009). Functional-coefficient models for nonstationary time series data. Journal of Econometrics 148, 101-113.

Chan, N. H. and Wei, C. Z. (1988). Limiting distributions of least squares estimates of unstable autoregressive processes. Annals of Statistics 16, 367-401.

Chen, B. and Hong, Y. (2012). Testing for smooth structural changes in time series models via nonparametric regression. Econometrica $80,1157-1183$.

Cheng, X. and Phillips, P. C. B. (2009). Semiparametric cointegrating rank selection. Econometrics Journal 12, 83-104.

Engle, R. and Granger, C. W. J. (1987). Cointegration and error correction: representation, estimation and testing. Econometrica 55, 251-276.

Fan, J. and Gijbels, I. (1996). Local Polynomial Modelling and Its Applications. Chapman and Hall, London.

Gao, J. and Phillips, P. C. B. (2013). Semiparametric estimation in triangular simultaneous equations with nonstationarity. Journal of Econometrics 176, 59-79.

Giraitis, L., Kapetanios, G. and Yates, T. (2014). Inference on stochastic time-varying coefficient models. Journal of Econometrics 179, 46-65. 
Johansen, S. (1991). Estimation and hypothesis testing of cointegration vectors in Gaussian vector autoregressive models. Econometrica $59,1551-1580$.

Karlsen, H. A., Myklebust, T. and Tjøstheim, D. (2007). Nonparametric estimation in a nonlinear cointegration type model. Annals of Statistics 35, 252-299.

Li, D., Phillips, P. C. B. and Gao, J. (2016). Uniform consistency of nonstationary kernel-weighted sample covariances for nonparametric regression. Econometric Theory 32, 355-385.

Li, D., Phillips, P. C. B. and Gao, J. (2019). Supplement to: 'Kernel-based inference in time-varying coefficient cointegrating regression'. Online Supplementary Document.

Li, K., Li, D., Liang, Z. and Hsiao, C. (2017). Estimation of semi-varying coefficient models with nonstationary regressors. Econometric Reviews 36, 354-369.

Lütkepohl, H. (2006). New Introduction to Multiple Time Series Analysis. Springer.

Park, J. Y., and Hahn, S. B. (1999). Cointegrating regressions with time varying coefficients. Econometric Theory 15, 664-703.

Park, J. Y., and Phillips, P. C. B. (1988). Statistical inference in regression with integrated processes: Part 1. Econometric Theory 4, 468-497.

Park, J. Y., and Phillips, P. C. B. (1989). Statistical inference in regression with integrated processes: Part 2. Econometric Theory 5, 95-131.

Park, J. Y. and Phillips P. C. B. (2001). Nonlinear regressions with integrated time series. Econometrica 69, 117-161.

Phillips, P. C. B. (1988). Multiple regression with integrated time series. Contemporary Mathematics 80, 79-105.

Phillips, P. C. B. (1991). Optimal inference in cointegrated systems. Econometrica 59, 283-306.

Phillips, P. C. B. (1995). Fully modified least squares and vector autoregression. Econometrica 63, 1023-1078.

Phillips, P. C. B. (1996). Econometric model determination. Econometrica 64, 763-812.

Phillips, P. C. B. (2007). Regression with slowly varying regressors and nonlinear trends. Econometric Theory 23, 557-614.

Phillips, P. C. B. and Durlauf, S. N. (1986). Multiple time series regression with integrated processes. Review of Economic Studies 53, 473-496.

Phillips, P. C. B. and Hansen, B. (1990). Statistical inference in instrumental variables regression with I(1) processes. Review of Economic Studies 57, 99-125.

Phillips, P. C. B., Li, D. and Gao, J. (2017). Estimating smooth structural changes in cointegration models. Journal of Econometrics 196, 180-195.

Phillips, P. C. B. and Ouliaris S. (1990). Asymptotic properties of residual based tests for cointegration. Econometrica 58, 165-193.

Phillips, P. C. B. and Perron, P. (1988). Testing for a unit root in time series regression. Biometrika 75, 335-346.

Phillips, P. C. B. and Solo, V. (1992). Asymptotics for linear processes. Annals of Statistics 20, 971-1001.

Robinson, P. M. (1989). Nonparametric estimation of time-varying parameters. Statistical Analysis and Forecasting of Economic Structural Change (ed. by P. Hackl). Springer, Berlin, pp. 164-253.

Toda, H. Y. and Phillips, P. C. B. (1993). Vector autoregressions and causality. Econometrica 61, 1367-1393.

Vogt, M. (2012). Nonparametric regression for locally stationary time series. Annals of Statistics 40, 2601-2633.

Wang, Q. and Phillips, P. C. B. (2009a). Asymptotic theory for local time density estimation and nonparametric cointegrating regression. Econometric Theory 25, 710-738. 
Wang, Q. and Phillips, P. C. B. (2009b). Structural nonparametric cointegrating regression. Econometrica 77, $1901-1948$.

Xiao, Z. (2009). Functional-coefficient cointegrating regression. Journal of Econometrics 152, 81-92.

Zhang, T. and Wu, W. B. (2012). Inference of time varying regression models. Annals of Statistics 40, 1376-1402.

Zhou, Z. and Wu, W. B. (2010). Simultaneous inference of linear models with time varying coefficients. Journal of the Royal Statistical Society Series B 72, 513-531. 